University of Rhode Island

DigitalCommons@URI

Open Access Dissertations

1995

\title{
An Investigation of the Material and Process Variables in Pharmaceutical Wet Granulation
}

George Sienkiewicz

University of Rhode Island

Follow this and additional works at: https://digitalcommons.uri.edu/oa_diss

\section{Recommended Citation}

Sienkiewicz, George, "An Investigation of the Material and Process Variables in Pharmaceutical Wet Granulation" (1995). Open Access Dissertations. Paper 457.

https://digitalcommons.uri.edu/oa_diss/457

This Dissertation is brought to you for free and open access by DigitalCommons@URI. It has been accepted for inclusion in Open Access Dissertations by an authorized administrator of DigitalCommons@URI. For more information, please contact digitalcommons-group@uri.edu. 
SHORTENED TITLE

\section{AN INVESTIGATION OF WET GRANULATION}


AN INVESTIGATION OF THE MATERIAL AND PROCESS VARIABLES IN PHARMACEUTICAL WET GRANULATION

BY

GEORGE SIENKIEWICZ

A DISSERTATION SUBMITTED IN PARTIAL FULFILLMENT OF THE REQUIREMENTS FOR THE DEGREE OF DOCTOR OF PHILOSOPHY

IN

PHARMACEUTICAL SCIENCES

UNIVERSITY OF RHODE ISLAND 1995 


\section{DOCTOR OF PHILOSOPHY DISSERTATION}

OF

GEORGE SIENKIEWICZ

APPROVED:

Dissertation Committee Major Professor
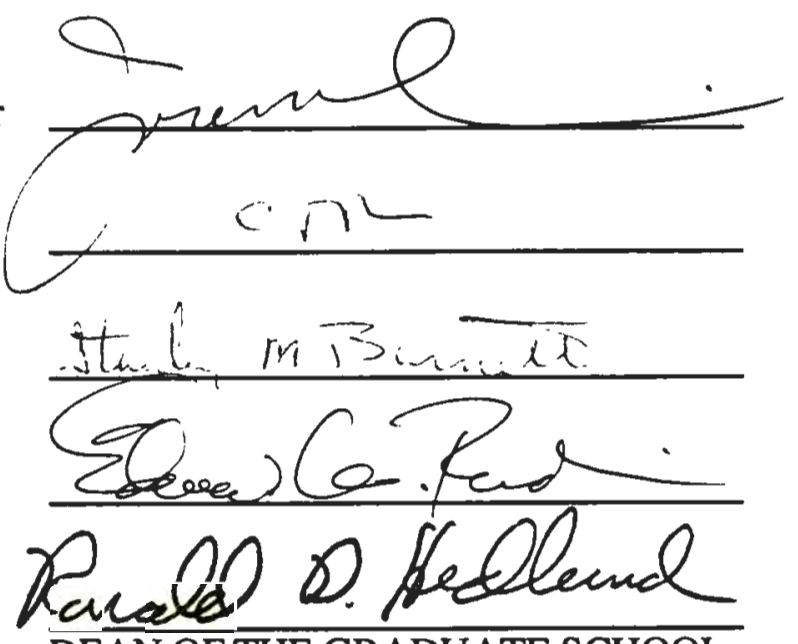

DEAN OF THE GRADUATE SCHOOL

UNIVERSITY OF RHODE ISLAND 
ABSTRACT

The effects of process and formulation variables on pharmaceutical wet granulation processes using various mixer-processors were studied. The first investigation utilized RSM methods with a central-composite experimental design to study two process variables using a double-paddle planetary mixer. Systematic numerical grid search procedures were used to locate an optimum combination of mixing speeds and granulating fluid levels which simultaneously satisfied all imposed constraints using multiple response variables. This optimum defined a granulation end-point which was an intermediate between a directcompression blend and a fully granulated product. Mixing speed played only a minor role in the process, which depended chiefly on the amount of fluid added. In a second investigation, a factorial design approach was used to discriminate between alternative high-shear mixer designs and two comparable pregelatinized starches by using high mixing speeds as a process variable. One mixer, the Ross PowerMix ${ }^{\mathrm{TM}}$, was found to have a significantly more intense mixing action than the TK Fielder ${ }^{\mathrm{TM}}$ which resulted in enhanced material and tableting properties. It was found that Starch $1500^{\mathrm{TM}}$ slightly outperformed Starch $1551^{\mathrm{TM}}$ in flow and compactability into tablets for a $90 \%$ acetaminophen-starch powder. A subsequent investigation used the PowerMix ${ }^{\mathrm{TM}}$ to study two additional process factors using the same acetaminophen-starch formulation. The interactions between mixing speeds, granulating fluid levels and starch types revealed that mixing speed was the critical variable in the process, followed closely by the amount of granulating fluid added. Differences between the two starches were substantiated. The effects of drying granulations by fluidized-bed techniques or by static oven drying were negligible. A fourth investigation tested the spheronization potential of three grades of microcrystalline cellulose with three grades of theophylline in a novel fluidized-bed rotary granulation technique. The coarsest grade of theophylline was amenable to processing using all three Avicels at drug 
loading levels of up to $90 \%$. The finest grade (micronized) formed acceptable spheres only at drug loading levels below $50 \%$. Larger grades of Avicel performed lightly better than the finest grade. Shape analyses revealed that sphere quality always deteriorated when drug loading levels exceeded $70 \%$. 


\section{ACKNOWLEDGEMENTS}

I am grateful to my major professor, Associate Dean Joan M. Lausier, Ph.D. for her years of patience when I had none, her encouragement when I needed more, her scientific insight when it was required, and her 'can-do' attitude which seemed to made all things possible. Thank you for your guidance and support!

My special gratitude to Dr. Christopher T. Rhodes, $\mathrm{Ph} . \mathrm{D}$., whose many efforts and dedication to my graduate school experience afforded me opportunities to conduct research and obtain funding that have made my experience memorable and rewarding. His guidance and assistance was always greatly appreciated.

I am greatly indebted to Dr. Edward M. Rudnic, whose selfless interest in the University and in furthering my research interests provided me with an opportunity to conduct my pelletization project at Schering-Plough Research in Miami, FL for two years.

I thank Dr. Stanley Bamett for serving on my committee and for disseminating his expertise both in class and whenever called upon.

I would like to thank Dr. Rosenbaum for her cooperation in serving during my qualifying and comprehensive examinations, as well as my dissertation defense.

I extend my gratitude to Dr. W. D. Lawing for his efforts in serving on my examination committees, but mostly for instilling in me an appreciation of statistical methods. 
I am grateful to Mr. Doug Cohen and Charles Ross \& Son, Co. for their years of financial support and for supplying me the Double Planetary Mixer and PowerMix.

I am grateful to Dr. Stu Porter at Colorcon, Inc. for allowing me to conduct research at their West Point, PA facility, and for their financial support.

My special thanks to my great friends Emilio Squillante and Pardeep Sethi, whose shared scientific inquiry (usually over Chinese food) made my University experience what a University experience ought to be: rich and profound.

'To all my other friends at the University, Alex Chueh, Karl Symecko and Polireddy Dondetti, my thanks for being around and commiserating.

My very special thanks to Denise Gorenski, whose ebullient manner, like a tide, could lift all boats, and Reba Whitford, who made URI 'home'.

I would like to thank all my other friends at the University, especially Dr. K, whose depth of scientific inquiry and dedication to teaching did not go unnoticed or unappreciated.

Finally, my everlasting thanks to my parents for their patience and support, who at times suffered through my degree more than I. 
PREFACE

This dissertation is prepared according to the manuscript format. The first manuscript investigates the use of a novel, dual-planetary mixer design for wet granulation purposes. It is the purpose of this investigation to explore the possibility that such a design can be considered a 'high-speed' or high-shear device, based on known principles of agglomerate growth. At the same time, the goal is to implement the methods of numerical optimization to 'map' the effects of process conditions on multiple response variables, thereby locating a prospective 'end-point' for the process. This work was presented at the Interphex 1993 Conference and Exhibition. Manuscript II uses two mixers understood to be of the high-shear type; their effects on the kinetics of particle growth can be contrasted with each other to differentiate their capabilities, as well as with the results of Manuscript I. The investigation focuses on the precise nature of the interactions between material and process variables in an effort to resolve doubts concerning excipient performance. This work will be presented at the Interphex 1995 Conference and Exhibition. Manuscript III builds upon Manuscript II, isolating the PowerMix processor as the higher intensity device. Its intense mechanical actions resolve more clearly the sometimes interacting effects of process and formulation factors, and allow the introduction of two more variables into the process for study (the effects of water addition and the method of water removal). Manuscript IV utilizes another novel high-shear method of granulation to produce specialized, spherical drug-loaded pellets. The investigation further isolates and explores the intimate interactions of pharmaceutical materials that may take place in this unique method of agglomeration. This work has been presented at the 1991 Conference of the American Association of Pharmaceutical Scientists. 


\section{TABLE OF CONTENTS}

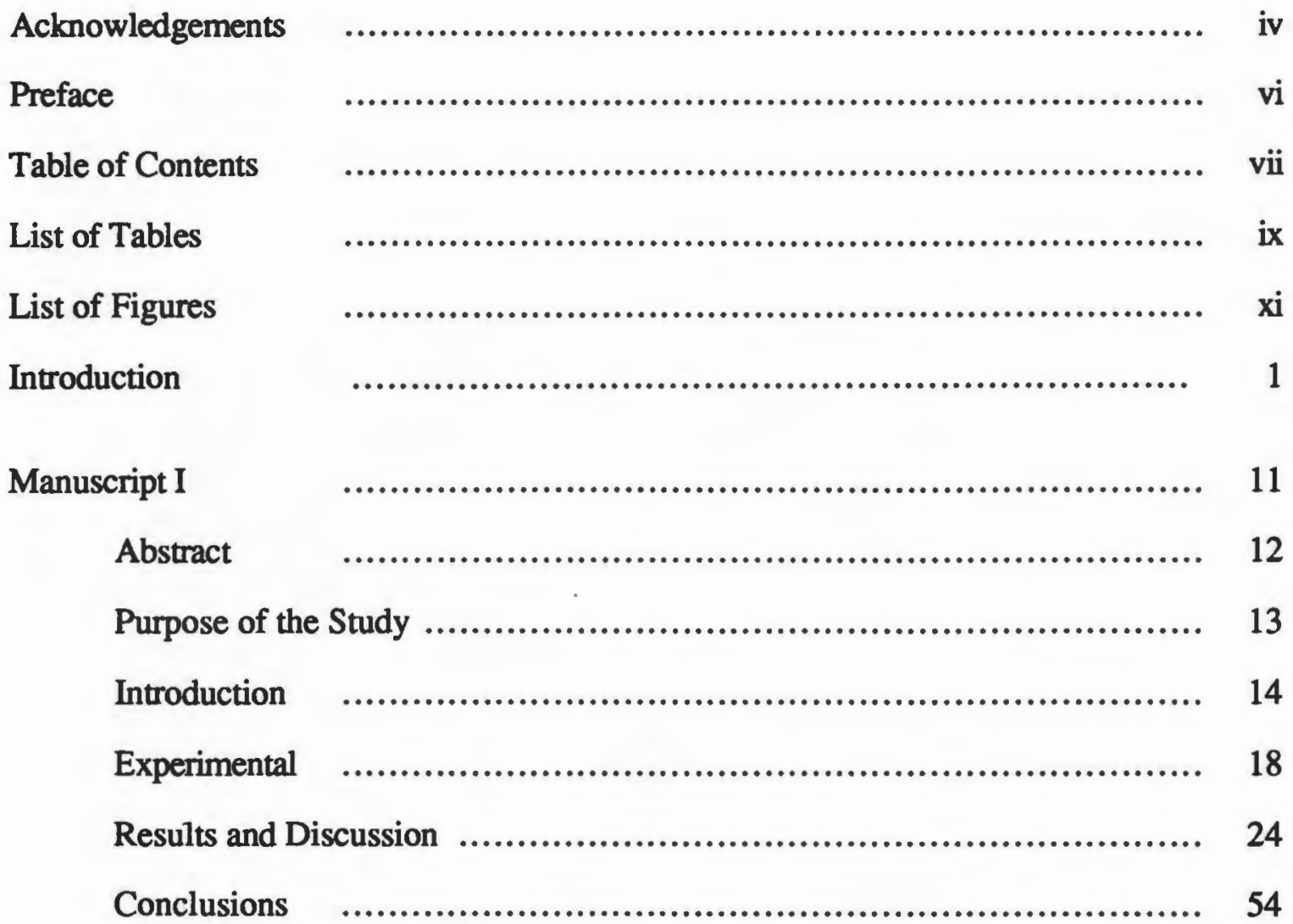

Manuscript II $\quad$................................................................. 60

Abstract $\quad$.................................................................. 61

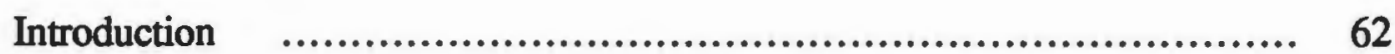

Experimental $\quad$................................................................. 64

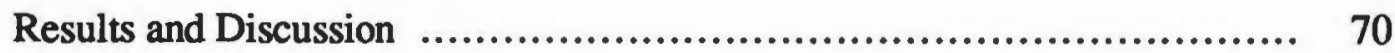

Conclusions $\quad$.............................................................. 97

Manuscript III $\quad$.............................................................. 100

Abstract $\quad$................................................................. 101

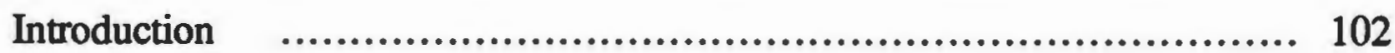


Experimental

Results

Discussion

Conclusions

Manuscript IV

Abstract

Introduction

Experimental

Results

Discussion

Conclusions 


\section{LIST OF TABLES}

Manuscript I

Page

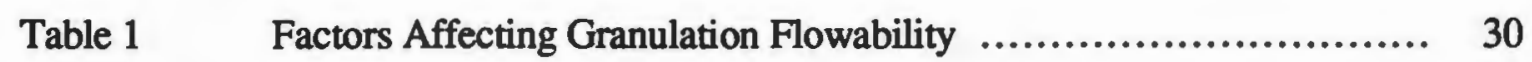

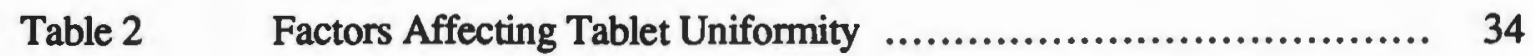

Table 3 Areas of Best Tablet Uniformity versus Water Level ................. 35

Table $4 \quad$ Optimization Constraints ............................................ 51

Table $5 \quad$ Optimum Factor Levels ....................................... 52

Manuscript II

Table 1 - Duncan's Multiple Range Test for Median Particle Size of Starches $\quad . .72$

Table 2 Duncan's Multiple Range Test for Geometric Mean Size of

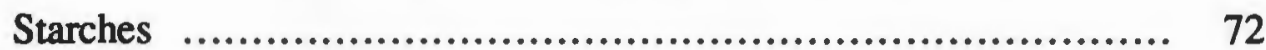

Table 3 Duncan's Multiple Range Test for Volume-Surface Mean Particle

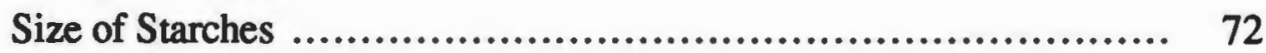

Table 4 Duncan's Multiple Range Test for Geometric Mean Particle Size

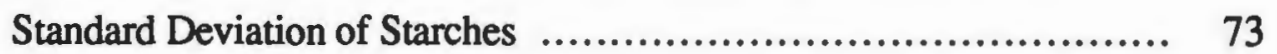

Table 5 deleted

Table 6 Mean Standard Deviations of the Geometric Mean Particle Size

(Particle Size Uniformity for Two Mixers at Two Speeds ............. 78

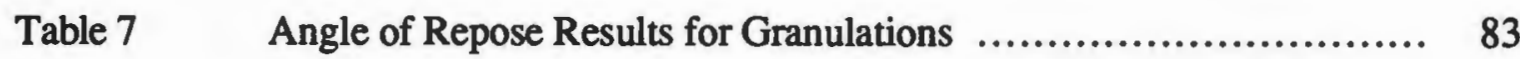

Manuscript III

Table $1 \quad$ Flowability Results: Angle of Repose and Compressibility Index ..... 119 


\section{Manuscript IV}

Table 1 Size Uniformity of $50 \%$ Avicel-Theophylline Spheres .............. 152

Table 2 Shape and Shape Uniformity of 50\% Avicel-Theophylline Spheres ... 154

Table 3 Compressibility Index of 50\% Avicel-Theophylline Spheres ......... 156

Table 4 Friability of $50 \%$ Avicel-Theophylline Spheres $\ldots . . . . . . . . . . . . . .158$

Table 5 Effect of Raw Material Grade on Theophylline Content $\ldots . . . . . . . . . . .161$

Table 6 Effect of Pellet Size Fraction and Percent Drug Loading on

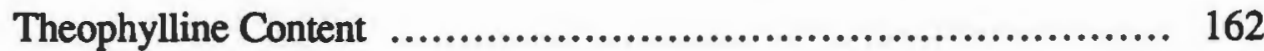

Table 7 Effect of Drug Loading on Pellet Shape Factor ...................... 166 


\section{LIST OF FIGURES}

Manuscript I

Page

Figure 1 Granulation Geometric Mean Particle Size .......................... 27

Figure 2 Granulation Particle Size Uniformity $\ldots . . \ldots \ldots \ldots \ldots \ldots \ldots \ldots \ldots \ldots \ldots . . . \ldots$

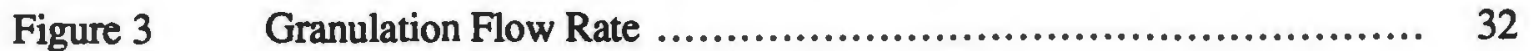

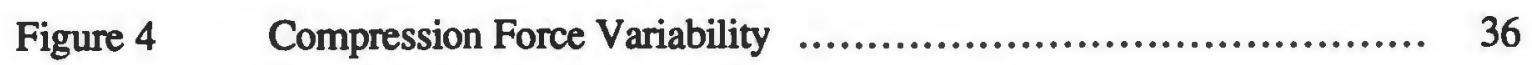

Figure 5 Tablet Thickness Uniformity .................................... 37

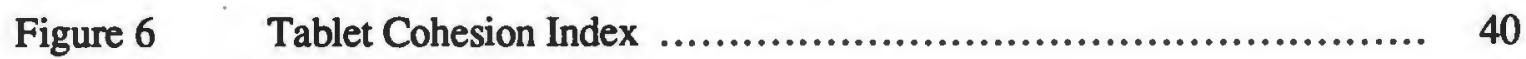

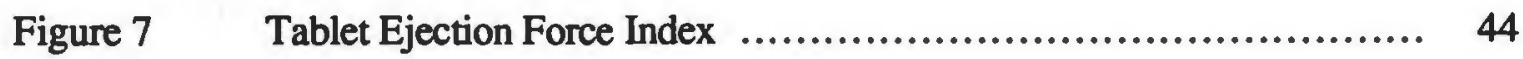

Figure 8 Surface-Volume Mean Particle Size.............................. 45

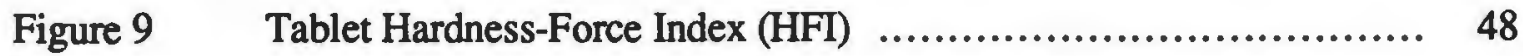

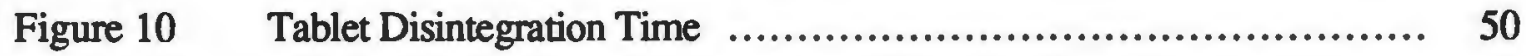

Figure 11 Geometric Mean Particle Size with Optimal Factor Levels ........... 53

Manuscript II

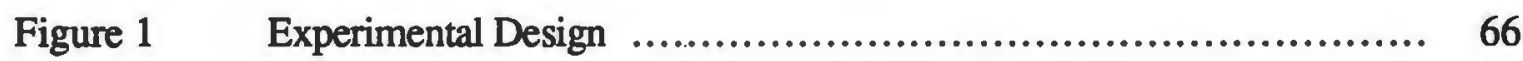

Figure $2 \quad$ Particle Size Analyses of Starch 1500 and Starch 1551 ............... 74

Figure 3 Surface-Volume Mean Diameter of Granulations Made With Two

Mixers and Two Mixing Speeds ................................... 77

Figure 4 Particle Size Distributions of Granulations Made With Two Mixers

at Two Mixing Speeds ....................................... 80

Figure 5 Tapped Bulk Density of Granulations for Two Mixers and Two Mixing

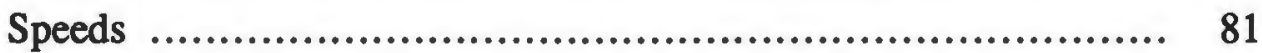

Figure 6 Graph of Angle of Repose vs. Compressibility Index ............... 84 
Figure 7 Flowability of Granulations (Angle of Repose)

Figure 8 Tablet Cohesion Index for Two Mixers and Two Mixing Speeds ......

Figure 9 Ejection Force and Surface-Volume Diameter for Two Mixers versus Chopper Speed ........................................ 89

Figure 10 Compression Force Variability versus Tablet Weight Variation ....... 91

Figure 11 Compression Peak Force Variability for Two Mixers

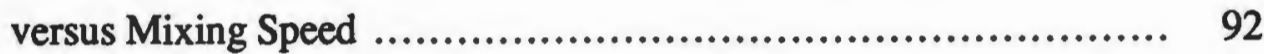

Figure 12 Tablet Friability for Two Mixers and Two Mixing Speeds ............. 94

Figure 13 Disintegration Time of Tablets versus Tablet Friability ................ 95

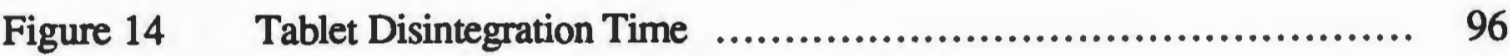

Manuscript III

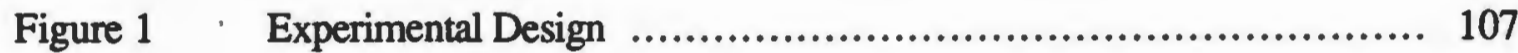

Figure 2 Granule Volume-Surface Mean Diameter ............................ 114

Figure 3 Standard Deviation of the Geometric Mean Particle Size .............. 115

Figure 4 Compressibility Index for Two Starches at Two Mixing Speeds ...... 117

Figure 5 Granulation Flowability for Two Starches at Two Mixing

Speeds and Two Water Levels .................................... 120

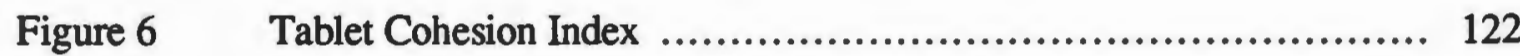

Figure 7 Ejection Force versus Tablet Crushing Strength ..................... 124

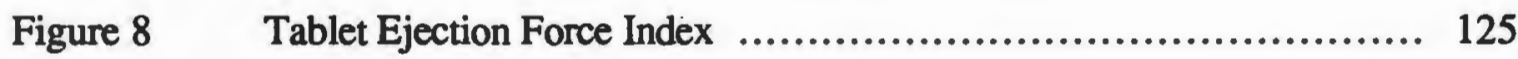

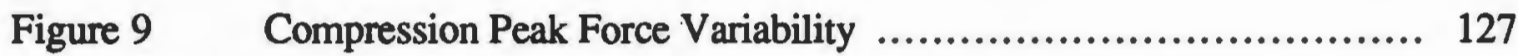

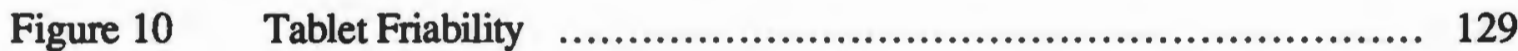

Figure 11 Tablet Disintegration Time for Two Starches at Two Mixing

Speeds and Two Water Levels ..................................... 131 
Manuscript IV

Figure 1 Particle Size Distributions of Three Avicel Grades ................... 147

Figure 2 Shape Factor Distributions of Three Avicel Grades ................... 148

Figure 3 Arithmetic Mean Particle Size of Raw Materials ....................... 149

Figure $4 \quad$ Particle Shape Factors of Raw Materials ........................... 150

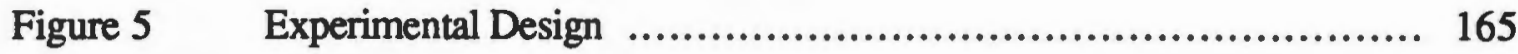


INTRODUCTION 
Pharmaceutical wet granulation is a process of size enlargement which is carried out for the purpose of altering any of several properties of the starting materials. Appelgren (1985) lists ten common reasons (such as flow, density, binding properties, etc.) for the application of these procedures in pharmaceutical dosage form development. It is, therefore, a routine operation in the manufacture of solid dosage forms, such as tablets and capsules.

Unfortunately, wet granulation remains somewhat of an art. It remains, despite a large body of scientific work, a partly subjective enterprise. Critical aspects of the process, such as when the 'end-point' has been reached, often rely on judgement, not science. As a result, disagreement can exist about the optimal process conditions, even among experienced and trained personnel.

The situation has been complicated somewhat by the introduction of newer types of equipment (Sienkiewicz, 1991), which have supplanted some older methods by carrying out processes at higher speeds and within a single unit operation. Previously, wet granulation was typically carried out using a number of time-consuming steps. With the introduction of high-speed equipment, 'one-pot' processors and other novel equipment designs, the entire operation can take place within a few hours and greatly reduce the number of processing steps. But these newer approaches also magnify potential problems in process control, not aided by the fact that the product is no longer visible or accessible during the process. It is, therefore, necessary to identify those factors which influence the process and to control them adequately.

Recent trends within the pharmaceutical industry have also made requisite a greater understanding of pharmaceutical manufacturing processes and greater precision in controlling important variables. The first is the concept of "validation", now mandated by the Food and Drug Administration as part of the development process for new drug products. Validation, in essence, proves that a manufacturing process does what it 
purports to do (Lachman and Lieberman, 1986) and consists of demonstrating that controlling critical steps in a process results in repeatable attributes. Validation procedures establish an acceptable range within which a given process variable is allowed to fluctuate. When that variable falls outside the established range, it is incumbent upon the manufacturer to demonstrate that the product has not been adversely affected. It is therefore necessary to quantify the effects of certain variables upon the process and the downstream products, and even to provide evidence for how the products would be affected should the process be carried out at a new and untested set of conditions.

Further impetus has been given in recent years to a more scientific approach to pharmaceutical processes such as wet granulation by the developing regulatory policies for the scale-up of batch size. As products evolve from the developmental stage to production scale (or undergo technology transfer), it is important that the quality and performance of the dosage form not be affected. Skelly et al. (1993) has recently outlined scale-up considerations for immediate-release oral solid dosage forms. Subsequently, similar recommendations were issued concerning the scale-up of extended-release oral dosage forms (Skelly et al. 1993). Both reports indicate the need for determining the operating principles of the equipment employed, as well delineating key parameters for the process changes which affect scale-up.

Pharmaceutical manufacturing processes are a marriage of machine and materials. It is difficult to consider machine or process variables without a knowledge of how drug materials may be affected, and vice versa. Since drug products must meet high standards for consistency and uniformity in all their physical and physicochemical attributes, the intimate relationship between a formulation and the physical transformations it must undergo to become a drug product must be very well characterized. As part of this realization, the pharmaceutical industry now devotes much more effort to the 
characterization and validation of excipient materials. Whereas the importance of process validation was recognized early on, the role of the variability in excipient materials is only now being actively addressed (Staniforth 1988 and Brittain 1987). Compendial standards for these materials focus on identity, purity, microbial limits, and other qualities, but the functionality of excipient materials is associated with their physical characteristics, not their chemical qualities (Shangraw 1981 and Chowhan 1993). As a result, much research now focuses on the specific differences, if any, between various types of celluloses, starches, sugars and the other commonly used ingredients in solid dose formulations. Differences in particle size, size distribution, shape, crystallinity, polymorphic forms, surface area, etc. have long been known to affect pharmaceutical properties such as compactability, disintegration, flow and dissolution rate. But it was the these attributes of the pure drug material which were usually considered, not those of the 'inert' adjunctive or excipient materials.

In summary, the foundation which forms the basis for the manufacture of modern drug delivery systems is comprised of at least these three key elements, namely the equipment used, the process variables which must be controlled and the nature of the materials used. It is therefore the overall intention of this dissertation work to examine all three elements, separately and in conjunction with each other, to better define, characterize and quantitate their roles in the formation of pharmaceutical granulations by three distinct wet agglomeration methods.

The first goal, and the subject of Manuscript I, is to apply a collection of mathematical and statistical techniques known as response surface methodology (RSM) to a wet granulation process in order to define and quantitate critical variables and 'map' their effects over their usable range on the characteristics of the resulting materials. Using these techniques, it may be possible to determine, in absolute terms, the ability of the mixer 
under investigation to perform as a 'high-shear' mixer. The effects of each variable on the resulting products, as well as on the other variable(s), can be measured. In this way, a novel mixer design can be characterized for the purposes of pharmaceutical wet granulation, a prospective 'end-pont' can be found, and a means for predicting outcomes at untested conditions may be provided.

Where two mixer-processors are purported to be of the 'high-shear' type, it is hypothesized that they, too, can be differentiated through the testing and evaluation of the resulting granulations and other output variables. A basis for resolving the capabilities of such equipment may be found by observing fundamental aspects of the agglomeration process itself. Seminal works by Newitt and Conway-Jones (1958) on the mechanisms of agglomeration and Rumpf (1974) on the strength of agglomerates form the basis of the current understanding of this phenomenon. It is generally agreed that the wet granulation process, as it is typically carried out, is a balance between particle size enlargement and degradation. The dominance of one effect over the other depends on both material and process factors. Mixers which generate very little shear will demonstrate unbridled particle growth or "ball growth". Mixers which operate at higher speeds and have various cutting or chopping tools within the mixing chamber reduce particle size by breaking apart weak agglomerates through high-speed collisions and mechanical shearing forces. One such 'high-speed' mixer is the TK Fielder processor, a vertical mixer with bottom-mounted impeller blades and a wall-mounted chopper. Previous investigations by Holm (1987) using the Fielder mixer concluded that the impeller (not the chopper) causes densification of the material and narrows the particle size distribution. The chopper was assumed to be too small to have a comminuting effect. The primary function of the chopper (not the impeller) was to disrupt the uniform flow pattern of the mass. In a subsequent study, Schaefer (1988) compared the Fielder with the Diosna mixer and found that the Diosna was more 
able to densify granules through a more intense mixing action. In Manuscript II the Fielder processor is compared with a novel high-shear design, the Ross PowerMix. The PowerMix employs a high-speed chopper arm which circulates about the mixing chamber in a mutually orbiting fashion with a standard mixing paddle. The paddle and chopper purportedly work in a synergistic manner by 'feeding each other' material. By itself, the slow-moving paddle lacks the ability to perform as a 'high'shear' device. The high-speed chopper, as a result of its intensity and mobility, appears to function effectively as a sizereduction tool. It is hypothesized here that differences between mixers can be delineated by observing their effects on the particle size distributions in the course of a wet granulation process. Low-shear mixers will result in unbridled particle growth with wider particle distributions than higher shear mixers, which will display the ability to control particle growth and help narrow the size distributions through mechanical intervention.

Two of the most commonly used pharmaceutical materials, microcrystalline cellulose and modified corn starch, are investigated in the present work. Microcrystalline cellulose (MCC) has been used in pelletization processes because of its ability, when wetted, to form rounded, plastic particles. These have been investigated as drug carriers (Avicel Spheres, FMC Corp.). The formation of spherical drug-loaded particles has several advantages, such as enhanced flow properties. Although work has been done on drug-loaded spheres in the past, it utilized a laborious, multi-step process to manufacture them (Malinowski, 1975). Recently, however, fluid-bed equipment has been outfitted with a horizontal rotating waffle-type plate which can be used to form these spheres in a wet agglomeration process and to dry them concurrently (Jäger and Bauer 1982). This type of process has many of the typical advantages and disadvantages of high-speed wet granulation. The process is rapid, reaching completion within an hour using a single piece 
of equipment. On the other hand, it is difficult to control particle growth rates and is difficult to carry out with cohesive materials.

The purpose of this work is to develop a reproducible method for the production of drug-loaded spherical particles in order to test the relative abilities of three grades of MCC to function as spheronizing agents. Two of the three grades have been commercially available; the third is an experimental grade which differs substantially in both particle size and shape from the other two. Because of these considerable differences in size and morphology, the larger grades are claimed to have superior powder flow characteristics. The creation of spherical particles using the new spheronizing technique relies on a balance of gravitational, centrifugal and fluidizing forces which keep the agglomerates in contact with the spinning rotor plate. Conceivably, the differences between MCC grades could translate to differences in their abilities to agglomerate and hold drug particles, as well as to flow smoothly during processing. No studies to date have examined the relative abilities of these MCC grades to act in this capacity.

To investigate the MCC grades under a variety of test conditions, experiments will be conducted using three chemically identical but physically distinct anhydrous theophylline powders. This drug is often formulated as a controlled release dosage form using coated pellets or seeds. It is hypothesized that dramatic differences in particle size, size distribution and particle shape of the drug may have significant effects on the fluid-bed rotary spheronization process hitherto unrecognized, particularly when used in conjunction with different MCC grades.

Pregelatinized starch is a common ingredient of solid dosage forms because of its ability to function as a binder, tablet disintegrant and flow aid. It has been used in the manufacture of directly compressible acetaminophen powders by a number of patented methods (Vogel 1984, Salpekar 1987 and Finnan 1987). Two forms of pregelatinized 
starch are commonly used. Starch 1551 is a fully pregelatinized form, while Starch 1500 is a partially pregelatinized form. There is a perception within the field that, when subjected to a high-shear process, Starch 1500 is inferior to Starch 1551 as far as its binding ability is concerned. There is little evidence available to directly compare the two. Using a novel high-shear mixer, the Ross PowerMix, the present work attempts to test the hypothesis that the two starches are, in fact, the same. The starches, by being subjected to what is purportedly an intense mechanical mixing action, will be tested as to their intended abilities to function as flow aids and binders in a directly compressible acetaminophen product.

In summary, the goals of this experimental work were therefore as follows:

1) To identify and quantify the effects of factors thought to be critical during the wet granulation process using both conventional and novel processing technologies.

2) The techniques of optimization will be used to locate an optimum set of operating conditions, as well as a well-defined region of acceptability in order to better define a prospective 'end-point' for a given process.

3) This work attempts to better define the abilities several different pharmaceutical mixer-processor designs to function as either 'conventional' or 'high-speed' units, based upon their effects on the properties of the resulting granulations.

4) This work will investigate and attempt to quantify the differences, if any, between several chemically identical but physically distinct grades of drug and excipient materials when subjected to novel high-speed mixing processes. 


\section{REFERENCES}

Applegren C. (1985) Recent Advances in Granulation Technology and Equipment, Drug Development and Industrial Pharmacy, 11(2\&3), 725-741.

Chowhan Z. (1993) Excipients and Their Functionality in Drug Product Development, Pharmaceutical Technology, 17(9),72-82.

Finnan J.L., Lisa R.E., Schmidt D.N. (1987) Process for Preparing Spray Dried Acetaminophen Powder and the Powder Prepared Thereby, United States Patent $\# 4,710,519$.

Lachman L., Lieberman H.A., Kanig J.L. (1986) The Theory and Practice of Industrial Pharmacy, Third Edition, Lea \& Febiger, Philadelphia.

Malinowski H.J.,Smith W.E. (1975) Use of Factorial Design to Evaluate Granulations Prepared by Spheronization, Journal of Pharmaceutical Sciences, 64(10),1688-1692.

Newitt D.M., Conway-Jones J.M. (1958), Transactions of the Institution of Chemical Engineers, 36, 422.

Rumpf H. (974), Chemical Ingineering Technologie, 46, 1.

Salpekar A.M., Denton L.E. (1987) Direct Tableting Acetaminophen Compositions, United States Patent \#4,757,090. 
Shangraw R.F., Wallace J.W., Bowers F.M. (1981) Morphology and Functionality in Tablet Excipients for Direct Compression: Part II, Pharmaceutical Technology, 10, 11-19.

Sienkiewicz G., Lausier J.L., Rhodes C.T. (1991) Recent Advances in Pharmaceutical Mixing Technology, Proceedings of the Interphex 1991, Cahners, 185-192.

Skelly J.P., et al. (1993) Scale-Up of Immediate Release Oral Solid Dosage Forms, Pharmaceutical Research, 10(2) 313-316.

Skelly J.P., et al. (1993) Scaleup of Oral Extended-Release Dosage Forms, Pharmaceutical Research, 10(12) 1800-1811.

Staniforth J.N. (1988) Particle Engineering and Excipient Performance, Indian Journal of Pharmaceutical Sciences, 50(4), 213-220.

Vogel S.T. (1984) Directly Compressible Acetaminophen Granulation, United States Patent \# 4,439,453. 


\section{Manuscript I}

The Optimization of a Wet Granulation Process Using a Novel Double Planetary Mixer Design 


\section{ABSTRACT}

A new double planetary mixer was utilized for an experiment to determine whether it has any capability as a high-shear design. A central composite experimental design was used to test the effects of two quantitative variables, mixing speed and the amount of water used, upon a wet granulation process. Response variables included parameters gauging the physical properties of granulations and tablets, as well as the mechanical forces exerted during the tableting process.

The results show that the mixer used cannot be considered a high-shear design. Its mixing action could not generate enough shear to function as such, although mixing speed did modify the effects of varying water levels on particle size and compression force variability. Even so, its effect was often negative, since it could facilitate agglomeration, but not control it.

Water levels had a strong effect on most of the variables measured, with many responses showing significant linear and quadratic effects.

Seven respopnse variables were used in a brute-force optimization search procedure using SAS. Response surface methodology located combinations of mixing speeds and water levels (45 to $65 \mathrm{rpm}$ with 385 to $425 \mathrm{ml}$ of water) which satisfied all constraints.

A new parameter is proposed for use in a tableting operation. This Hardness-Force Index combines compactability and ejection properties into a single parameter. Its utility requires further study. 


\section{PURPOSE OF THE STUDY}

The purpose of this study is to study the effects of these two variables on a wet granulation process and in particular:

1) To identify and quantify the effects of water addition and mixing speed on the characteristics of the in-process materials, tableting parameters and finished products of a wet granulation process

2) To apply particle growth kinetics in order to test whether the Ross Double Planetary Mixer can be construed as a high-shear mixer

3) To apply response surface methodology for finding within the factor space the optimum combination of process variables

4) To develop and utilize single-parameter compression and ejection force data for locating optimum conditions

5) To propose the use of hardness-force index which incorporates both single parameter force indices (compression and ejection) into a single quantity

6) To suggest a more rational scientific approach, using optimization techniques, for finding the 'end-point' of a given wet granulation process which does not rely upon subjective means 


\section{INTRODUCTION}

A new mixer design, the Ross Double Planetary Mixer, has been introduced recently to the US market. Intended primarily for liquid mixing, its design nevertheless makes it appropriate for wet granulation. Its orbiting dual-paddle design purportedly allows the two blades to work in a synergistic fashion over a continuous range of speeds. This allows for mixing speed to be studied as a process variable at any speed over the available range of $0-95 \mathrm{rpm}$.

The function of the mixing tools in a wet granulation process, regardless of type, is to impart motion to the contents of the mixing chamber. Mixing efficiency is maximal when all of the material is in constant motion. This allows the uniform distribution of all of the materials throughout each other, whether solid or liquid. The amount of shear required to adequately distribute a binding fluid depends upon its rate of addition. Low rates of addition, or the addition of fluid in an already subdivided state (e.g. spraying), allows the use of low shear equipment, including fluid-bed granulators. As the fluid addition rate increases, or the fluid is added as in a 'bulk' state (e.g. pumped or 'dumped'), the mixerprocessor must supply additional shear in order to rapidly distribute the liquid. Timko (1987) found that high-shear mixers were capable of adequately distributing granulating fluids even when 'dumped' all at once. Ordinarily, however, binding fluids are pumped at a convenient rate so that the process can be carried out quickly, but without loss of control. In the present investigation, the binding fluid is added at a constant rate. This allows the process to be carried out successfully at any of the available mixing speeds.

It has been shown that the liquid requirement for given wet granulation process at high mixing speeds is lower than at lower speeds (Kristensen and Schaefer, 1987). This is due, in part, to high-speed collisions between particles and surfaces within the mixing chamber. As particles collide, the liquid surrounding each particle or contained within the 
moist granules is expressed. Intragranular porosity is reduced as the particles are brought closer together by impact. Hence, it should be possible to observe the effects of speed by the reduction in the amount liquid necessary to achieve a given state of agglomeration. The amount of liquid necessary to attain a certain particle size should be less when the mixer is operated at its highest speed (95 RPM) when compared with a low speed (35 RPM).

The mechanism of agglomeration has been described by Newitt and Conway-Jones (1958). As liquid is added with mixing to a bed of solid particles, the particles pass through several distinct states of agglomeration. Increases in van de Waals forces, surface tension and capillary forces combine to hold individual particles together as moist granules. Smaller agglomerates then combine with each other to grow exponentially in size. Usteri (1988) describes this relationship:

$$
\ln d_{50}=A+k \pi
$$

where $d_{50}$, the median particle size, is a function of the amount of granulating fluid added $\pi . A$ is of the same order as the logarithm of the initial diameter of the starting material. The slope, $k$, of the regression line is an estimate of the growth rate. Agglomerates cannot, of course, grow in size without bound. Beyond a certain maximum, the agglomerates become too large to be held together by weak forces. This maximum attainable size can been estimated (Kristensen and Schaefer, 1987). Thus the wet granulation process is usually a dynamic process of size enlargement coupled with simultaneous size reduction in which both material and process factors play an important role. The mixing tools of the processor which are responsible for initiating and propagating the agglomeration of smaller particles as liquid is added also facilitate the reduction in size of the largest moist agglomerates through a cutting or shearing action. The net effect of this dynamic balance is a narrowing of the particle size distribution, where 
the smallest particles become larger and the largest particles become smaller. Thus the intensity of the mixing action of a given processor can be described, at least in relative terms, by it ability to 'control' particle growth in this way. Whether the Ross Double Planetary Mixer has this capability to control particle size and narrow its distribution within its available range of mixing speeds is one purpose of the current investigation.

A critical aspect of the wet agglomeration process is establishing the moment at which the liquid addition and mixing should be terminated. That particular instant concludes the granulation stage of the process and signals the beginning of the drying and sizing operations. Termination of the wet agglomeration phase presumably results in a moistened bed of discrete particles which, after the entire process is completed, should result in dried granules of the appropriate size and size distribution. While there is a common understanding of the meaning of the term 'end-point' in describing this demarcation point, establishing precisely when it has been reached during a given operation is much less clear and continues to be somewhat of an art.

In an effort to bring about a more rational and scientific approach to end-point detection, much recent work has focused on characterizing the agglomeration process using indirect methods to measure the strength or other physical properties of the moistened mass. Efforts such as those by Cliff (1990) have better defined the end-point in terms of the load on the main impeller shaft in different mixers. Numerous other methods of indirectly characterizing the process have been attempted. These have been reviewed by Schwartz (1988) and more recently by Corvari, et al. (1992). While these efforts have been useful for describing the process in more concrete terms as it occurs, these indirect measurements do not, by themselves, locate the 'end-point'. This is accomplished by reconciling granulations with the desired attributes to the 'fingerprint' obtained by these indirect measurements. Future repetitions of the same granulation process are then facilitated by using the calibrated fingerprint as a guide. The original problem of locating 
the end-point which ultimately yields the best granulation still, however, remains. Isolating the conditions which yield the best results becomes instead a problem amenable to the techniques of optimization.

Techniques of optimization have become valuable tools for pharmaceutical purposes. Original works by Box (1954) have been adapted by Schwartz (1973) to study pharmaceutical systems using Lagrangian methods. These analytical methods become complex as more variables are added to the system, eventually making them impractical. Schwartz $(1973,1981)$ overcame this difficulty by applying computerization techniques to develop a formulation with optimum properties. Since those early works, the techniques of optimization have become more routine. Schwartz (1985) applied computer-aided optimization techniques to the optimization of a slow-release product. They have been applied to study wet granulation in high-shear mixers by Vojnovic $(1992,1993,1994)$ to optimize water addition, granulation time and mixing speed. Bohidar (1991) published segments of linear programming using SASß to facilitate computerized search procedures. Some of those programs have been adapted for use in the present study.

The chief goals of optimization in the present work are to, in a precise way, quantify the effects of water and mixing speed in this agglomeration process. The ability of the mixer to control particle growth can be assessed. In addition, response surface methodology will be invoked to 'map' response variables and thereby locate an optimum point among sometimes competing response variables. This point corresponds to the best combination of conditions for carrying out the process and will more precisely locate the 'end-point' of the granulation. 
EXPERIMENTAL

Materials

Dibasic Calcium Phosphate Dihydrate USP (Emcompress ${ }^{\circledR}$, Mendell, Carmel, NY) was available from the manufacturer. Lactose, Hydrous NF (Mallinckrodt Co.), Sodium Starch Glycolate (Explotab®), Mendell, Carmel, NY), Povidone USP K29/32 (Plasdone® K29/32, GAF Corp., Wayne, NJ) and Magnesium Stearate NF (Fisher Scientific) were available at the University. Acetaminophen (used as a tracer) was obtained from Mallinckrodt (Lot\#4899-2K-768).

Formula

\begin{tabular}{|l|c|c|}
\hline Ingredient & $\mathrm{mg} / \mathrm{tab}$ & $\%$ \\
\hline Acetaminophen USP & 4.00 & 1.00 \\
\hline Dibasic Calcium Phosphate Dihydrate USP & 188.00 & 47.00 \\
\hline Lactose, Hydrous NF & 188.00 & 47.00 \\
\hline Povidone USP & 8.00 & 2.00 \\
\hline Purified Water USP & $*$ & $*$ \\
\hline Sodium Starch Glycolate NF & 10.00 & 2.50 \\
\hline Magnesium Stearate NF & 2.00 & 0.50 \\
\hline Total & 375.000 & 100.00 \\
\hline
\end{tabular}

* Water is expelled during processing. 
METHODS

\section{Experimental Design}

The experimental scheme for this experiment uses a central composite design with two variables. Four center points and an $\alpha$ value of 1.414 were chosen for rotatability in accordance with criteria outlined by Box (1987). The coded factor levels are shown below. Seven additional runs were performed beyond the twelve required for a complete secondorder design. One additional trial (\#9) was conducted in the region suspected to be close to the optimum at $(-\alpha,-\alpha)$. Two additional runs were conducted at the center point $(0,0)$ and one additional replicate was conducted at each of the factorial points $(-1,-1),(-1,1),(1,-1)$ and $(1,1)$. Statistical analyses were performed using SAS (SAS Institute, Cary, NC, Release 6.08). Backward elimination regression analyses use 0.10 as the criteria for retention in the model (SLSTAY=0.10).

CENTRAL COMPOSITE EXPERIMENTAL DESIGN IN TWO DIMENSIONS

\begin{tabular}{|c|c|c|}
\hline TRIAL & $\mathrm{X}-1$ & $\mathrm{X}-2$ \\
\hline 1 & -1 & -1 \\
\hline 2 & -1 & 1 \\
\hline 3 & 1 & -1 \\
\hline 4 & 1 & 1 \\
\hline 5 & -1.414 & 0 \\
\hline 6 & 0 & 1.414 \\
\hline 7 & 1.414 & 0 \\
\hline 8 & 0 & -1.414 \\
\hline 9 & -1.414 & -1.414 \\
\hline 10 & 0 & 0 \\
\hline
\end{tabular}


Granulation

The Ross DPM Double Planetary Mixer was made available from Charles Ross \& Son Co., Inc. Hauppauge, NY.

Liquid was added during granulation by pumping at $60 \mathrm{ml} / \mathrm{min}$ using a Masterflex Peristaltic Pump \#7553-30. Drying was done in a circulating hot air oven (Thelco Tray Oven Model 28).

Milling operations were performed using a Stokes Oscillating Mill through a \#12 wire mesh screen. Lubrication with Magnesium Stearate was performed in a Turbula Blender.

\section{Particle Size Testing}

Sieve analyses were performed with approximately 20 -gram samples of dried granulate added to a stack of US Standard Sieves, dried and tared. Sieving was done using a Van-Kel Model 18480 Sieve Shaker for 5 minutes at a \#7 setting, or until no further change takes place. Analyses are performed in triplicate. Size parameters were calculated from the following formulas, where $n$ is the weight percentage of a size $d$, in microns:

$$
\begin{gathered}
\text { Geometric Mean Size } d_{\text {geo }} \\
\log d_{g e o}=\frac{\sum(n \log d)}{n}
\end{gathered}
$$


Geometric Mean Size Standard Deviation $\sigma_{g e o}$

$$
\log \sigma_{g e 0}=\sqrt{\frac{\sum\left[n\left(\log d-\log d_{g e 0}\right)^{2}\right]}{\sum n}}
$$

Volume-Surface Mean Particle Size $d_{v s}$

$$
d_{v s}=\frac{\sum n d^{3}}{\sum n d^{2}}
$$

\section{Bulk Density}

Free-flowing and tapped densities were determined by pouring $100 \mathrm{ml}$ samples through a funnel into a graduated cylinder, then tapped 1000 times. Volume determinations before and after tapping ( $v$ and $v_{o}$, respectively) a known weight of granulate are used to calculate a compressibility index, expressed as a percentage:

$$
\text { Compressibility }=\left[1-\frac{v}{v_{0}}\right] \cdot 100
$$


Flowability

Dried unlubricated granules are poured through a funnel into a $7 / 16 "$ round tableting die. Equally sized samples in five replicates are poured through the funnel. The time elapsed for all material to pass is the flow rate $(\mathrm{gm} / \mathrm{sec})$

Tableting

Tablets were compressed using an instrumented rotary tablet press (PennwaltStokes Model 900-512) operating at $30 \mathrm{rpm}( \pm 1)$ adjusted to three compression settings approximating $12 \mathrm{kN}, 16 \mathrm{kN}$ and $20 \mathrm{kN}$. The tablets are compressed using a single punch and die set (7/16" round, flat-faced, no bevel-edge) to a weight of $375 \mathrm{mg}$. During tableting, a minimum of thirty tablets are compressed. Each compression-ejection cycle is measured and recorded individually; means and standard deviations of each of the following parameters are generated by the data acquisition and analysis software:

1) Peak Compression Force

$(\mathrm{kN})$

2) Area Under Compression Force-Time Curve

(kN•msec)

3) Peak Ejection Force

4) Area Under Ejection Force-Time Curve

(N॰msec)

The tablet 'cohesion index' is calculated from the following formula:

$$
\text { Cohesion Index }=\frac{\text { Crushing Force }(\mathrm{kg})}{\text { Compression Force }(\mathrm{kN})} \times 10^{5}
$$




\section{Tablet Weight}

At least ten tablets are weighed indiviually on a Mettler AE 240 Digital Balance to an accuracy of \pm 0.00001 grams.

$$
R S D=\frac{\text { Sample Standard Deviation }}{\text { Sample Mean }} \times 100
$$

\section{Tablet Thickness}

The thickness of at least ten individual tablets is measured using an analog micrometer (Mitutoyo Analog Micrometer) accurate to \pm 0.0001 inches.

Tablet Hardness

The diametrical crushing strength of at least ten individual tablets is measured using the Erweka Tablet Hardness Tester Model TBT. Results are reported in kilograms.

Tablet Disintegration

The disintegration time of six individual tablets is measured using the USP Disintegration Apparatus, with water at $37^{\circ} \mathrm{C}$ as the medium. 
RESULTS AND DISCUSSION

Particle Size Analysis

Sieve analyses of dried, milled, unlubricated granulations are reduced to two size parameters, the geometric mean and volume-surface mean sizes. They are calculated directly using the formulas given (see Experimental, Particle Size).

The geometric mean particle sizes for each experimental trial is fitted with the following second-order equation using a backward elimination regression procedure.

$$
d_{g}=111.0+0.00123 \cdot V^{2}+0.00051 \bullet S \bullet V
$$

The overall regression is significant with an $r^{2}=0.97$. Analysis of variance reveals significant linear and quadratic effects and no significant lack of fit.

The most obvious feature of the geometric mean particle size (Figure 1) is its strong increase as water is added to the system. There is an upward curvature in the surface, which corresponds to a significant quadratic effect $(p<0.0001)$. Particle growth during the agglomeration process, then, occurs at a rate not merely proportional to the amount of water used, but to its square.

At high water levels, increasing mixing speed causes a significant growth in particle size. The same does not occur when less water is used. Statistically, this results in a significant interaction effect between speed of mixing and water levels $(p<0.0001)$.

A particularly useful size parameter, particularly when surface area is an important consideration, is the surface-volume (or volume-surface) mean diameter $d_{v s}$. According to Parrott (1970), a unit volume of monosized particles with a diameter $d_{v s}$ will have a total surface area identical with the surface of a unit volume of actual sample having a 
mean surface-volume diamter $d_{v s}$. Of course, subdividing the same unit volume of material into smaller pieces (decreasing $d_{\nu s}$ ) will greatly increase the amount of surface area. The unique value of the $d_{v s}$, then, is that the increase in surface area is proportional to the decrease in $d_{v s}$. The importance of specific surface in a wet granulation process has been investigated by Pendharkar, et al. (1990).

A regression analysis of $d_{\nu s}$ data based on the same sieve analyses also shows a quadratic increase in size $(p=0.0003)$. The interaction effect between MIXING SPEED and WATER LEVEL is less pronounced $(p=0.06)$, but the effect of MIXING SPEED alone is more significant $(p=0.01)$. Overall, $d_{g}$ data fits the regression model more closely than $d_{v s}$ data.

It is clear from particle size data that the Ross Double Planetary Mixer (DPM) itself seems to have only a minor, though still significant, influence on the progression of the agglomeration process. Over the available range of mixing speeds, it plays a much smaller role in the size enlargement process than does the amount of water used. Faster mixing speeds result in larger particles, particularly if more water is used. These observations are consistent with the idea that the function of the mixing blades is to distribute the granulating fluid throughout the mass. At higher speeds, the same amount of water is distributed more quickly, in effect initiating the agglomeration process earlier and 'lengthening' its duration, even though actual process time is unchanged.

The question of whether this mixer can be considered a high speed mixer can be resolved, in part, by considering what happens when a truly high-speed processor is used. Several such devices are currently in use for granulation purposes, and all utilize some sort of high-speed cutting or chopping tool in addition to a paddle or mixing blade. High speed mixers generally perform a dual role. On one hand, the high speed tool helps introduce material into the agglomeration process more quickly. Once agglomerates exceed 
a certain size, however, it functions to reduce them to smaller particles. In other words, the overall effect is to make small sizes larger while making large sizes smaller, thereby keeping the particle size distribution more narrow (uniform). This is generally considered an advantage which may result in smaller variations in subsequent processing steps.

Based on the results gathered from particle size data, the Double Planetary Mixer does not appear to function as a high-shear mixer. It does not control particle size, since even the highest mixing speeds serve only to increase particle size, not to decrease it. 
Figure 1

\section{Geometric Mean Particle Size (microns)}

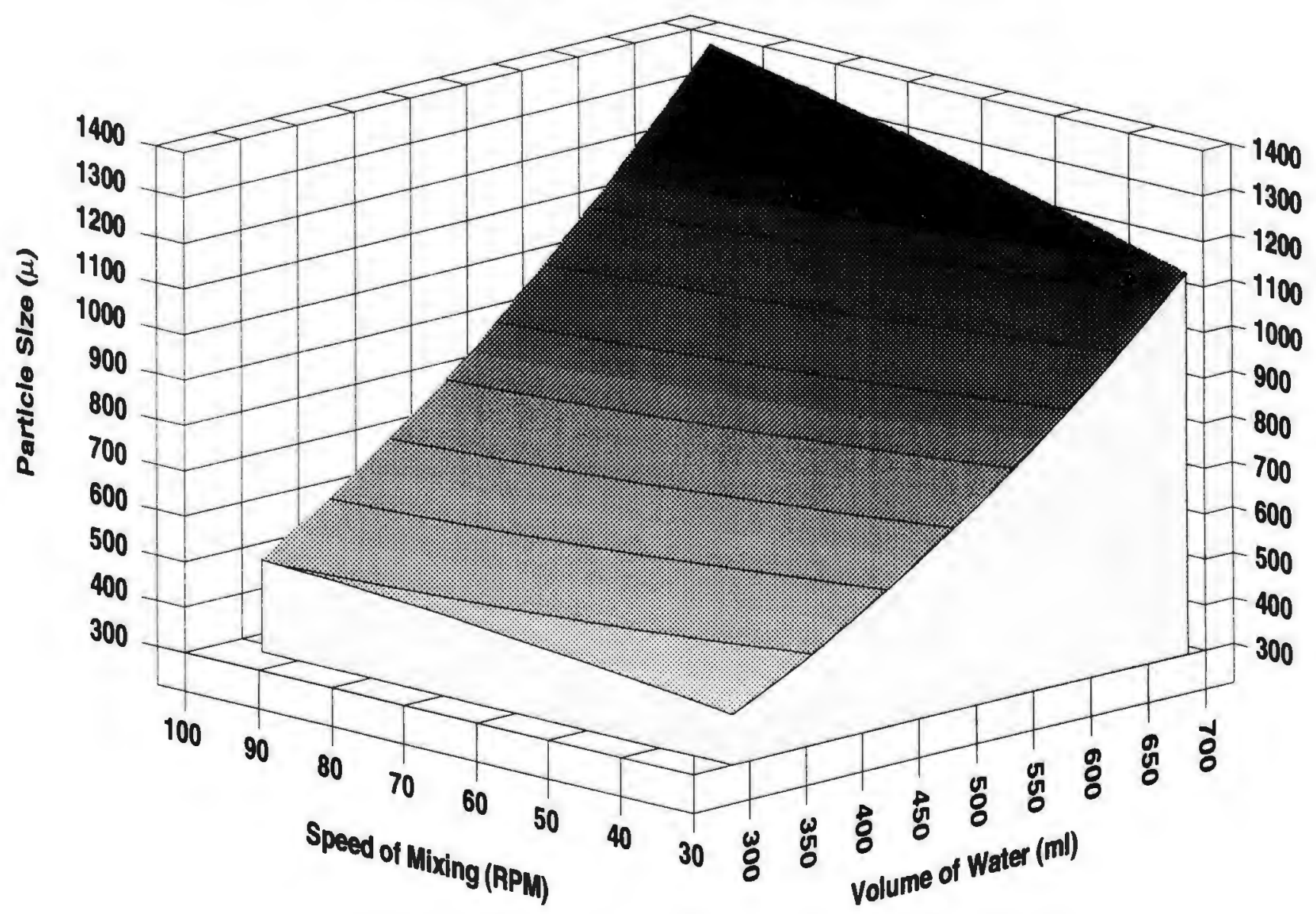


Granulation Uniformity

The uniformity of the particle size of a granulation can be expressed as the standard deviation of the geometric mean diameter. A response surface analysis of granulation uniformity as a function of MIXING SPEED and WATER LEVEL is a saddle configuration with no clear maximum or minimum value (Figure 2). Areas of greatest uniformity (smallest standard deviation) occur where both factors are at their highest or lowest levels simultaneously, as well as the diagonal region inbetween.

\section{Response Surface Regression Analysis}

$$
\sigma_{g e o}(\times 100)=146+0.27 \cdot S+0.13 \cdot V+0.0056 \cdot S^{2}-0.0024 \cdot S \cdot V+0.000036 \bullet V^{2}
$$

Significant Factors ( $p$ values) Affecting Particle Size Uniformity

\begin{tabular}{|l|c|c|c|c|c|}
\hline & Speed & Volume & Speed $^{2}$ & Speed•Volume & Volume $^{2}$ \\
\hline Uniformity $\left(\sigma_{\text {geo }}\right)$ & - & 0.017 & 0.038 & 0.024 & - \\
\hline
\end{tabular}

Granulation uniformity is not a strong function of either SPEED OF MIXING or WATER LEVEL. It is only weakly related to the main factors and their interactions, as indicated by the low $r^{2}$ statistic. The insensitivity to mixing speed supports the contention that the mixer is unable to control particle size. 
Figure 2

Granulation Particle Size Uniformity

(Relative Standard Deviation (RSD,\%) of Geometric Mean Size)

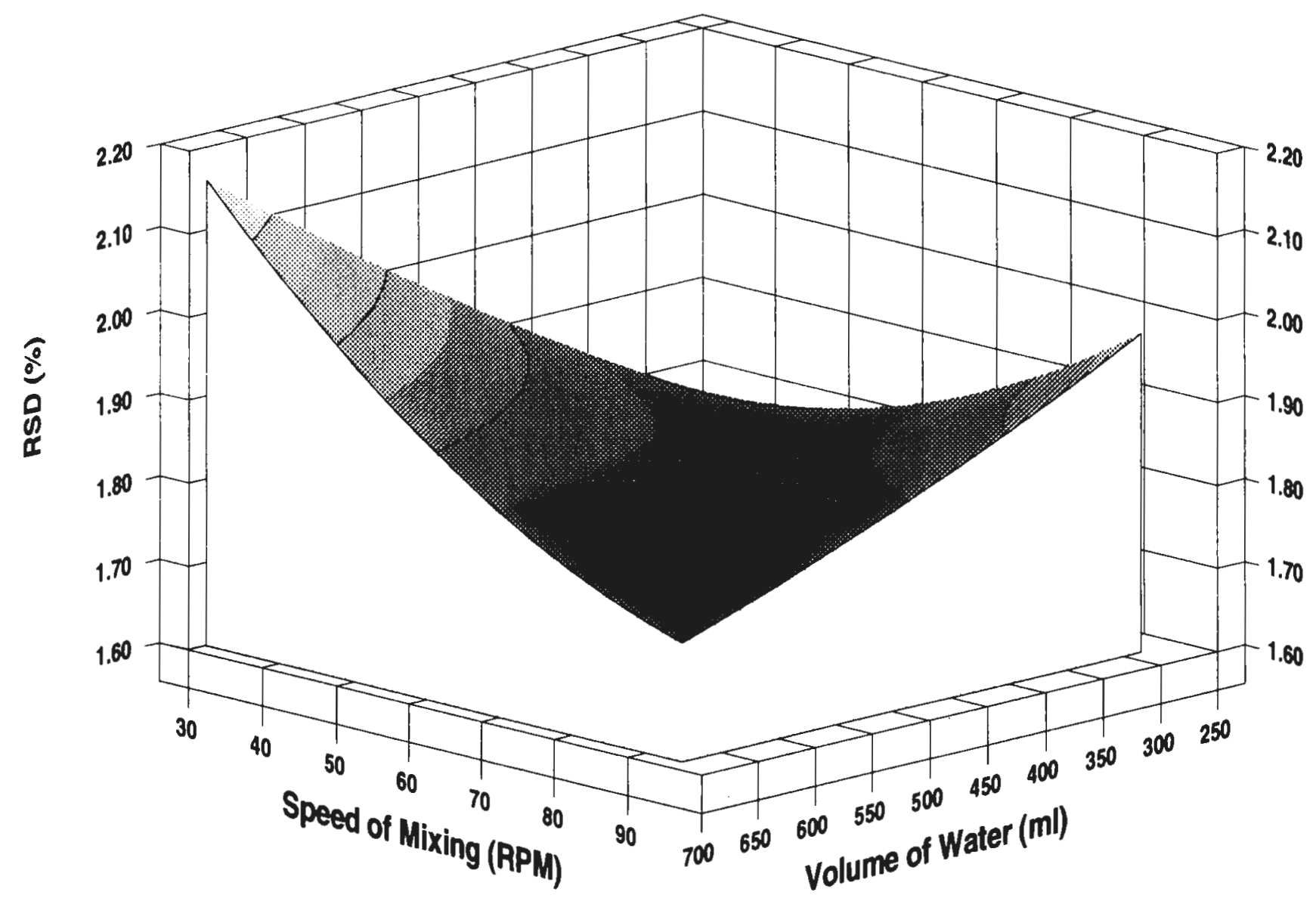


Granulation Flow

The flowability of granulations is important for maintaining smooth, uniform processing. Accurate die filling during tableting, for example, is of vital importance in pharmaceutical operations and requires both fast and reproducible flow characteristics. The flowability of solids has been measured by both static and dynamic methods; both can be used to indicate both flow rate and flow rate uniformity. Two separate measures have been used in this experiment, the compressibility index and the funnel flow rate.

The compressibility index, sometimes referred to as the Carr Index (Carr, 1965), uses bulk density measurements to indicate relative flowability. Expressed as a percent, values below $15 \%$ usually give rise to good flow characteristics. Values above $25 \%$ indicate poor flowability (Lachman and Lieberman, 1986).

The overall mean value for the compressibility index for all batches is $9.0 \%$, indicating superb flowability. Table 1 below examines the main and interaction effects on both the compressibility index and flow rate (' - ' indicates statistically not significant):

\section{Table 1}

Significant Factors ( $p$ values) Affecting Granulation Flowability as Measured by the Carr Compressibility Index and the Flow Rate Through a Die

\begin{tabular}{|c|c|c|}
\hline & Carr Index & Flow Rate \\
\hline & $r^{2}=0.75$ & $r^{2}=0.78$ \\
\hline Speed of Mixing (S) & - & - \\
\hline Water Level (V) & - & - \\
\hline Speed•Speed (S $\left.{ }^{2}\right)$ & - & - \\
\hline Water Water $\left(\mathrm{V}^{2}\right)$ & 0.0002 & 0.0001 \\
\hline Speed•Water $(\mathrm{SV})$ & - & - \\
\hline
\end{tabular}


Both sets of data are fit equally well by the regression equations ( $r^{2}$ is similar). Results show both measures of flowability are strong functions of the square of the amount of water used. Figure 3 shows the response surface for flow rate. Neither is related to any significant degree to mixing speed.

Interestingly, the expected inverse proportionality between the compressibility index and the flow rate (a low index leading to a high flow rate) is not evident here. Instead, there is a direct proportionality between the two. This apparent anomalous result occurs because larger granules encounter difficulty passing through the die orifice for a reason unrelated to flowability per se. Coarse particles tend to interlock, forming loose aggregates large enough to 'bridge' the die opening. Even assuming that there is no difference in surface roughness (there is no reason to believe there is one), it takes fewer large particles to form such an obstruction. 
Figure 3

\section{Granulation Flow Rate (gm/sec)}

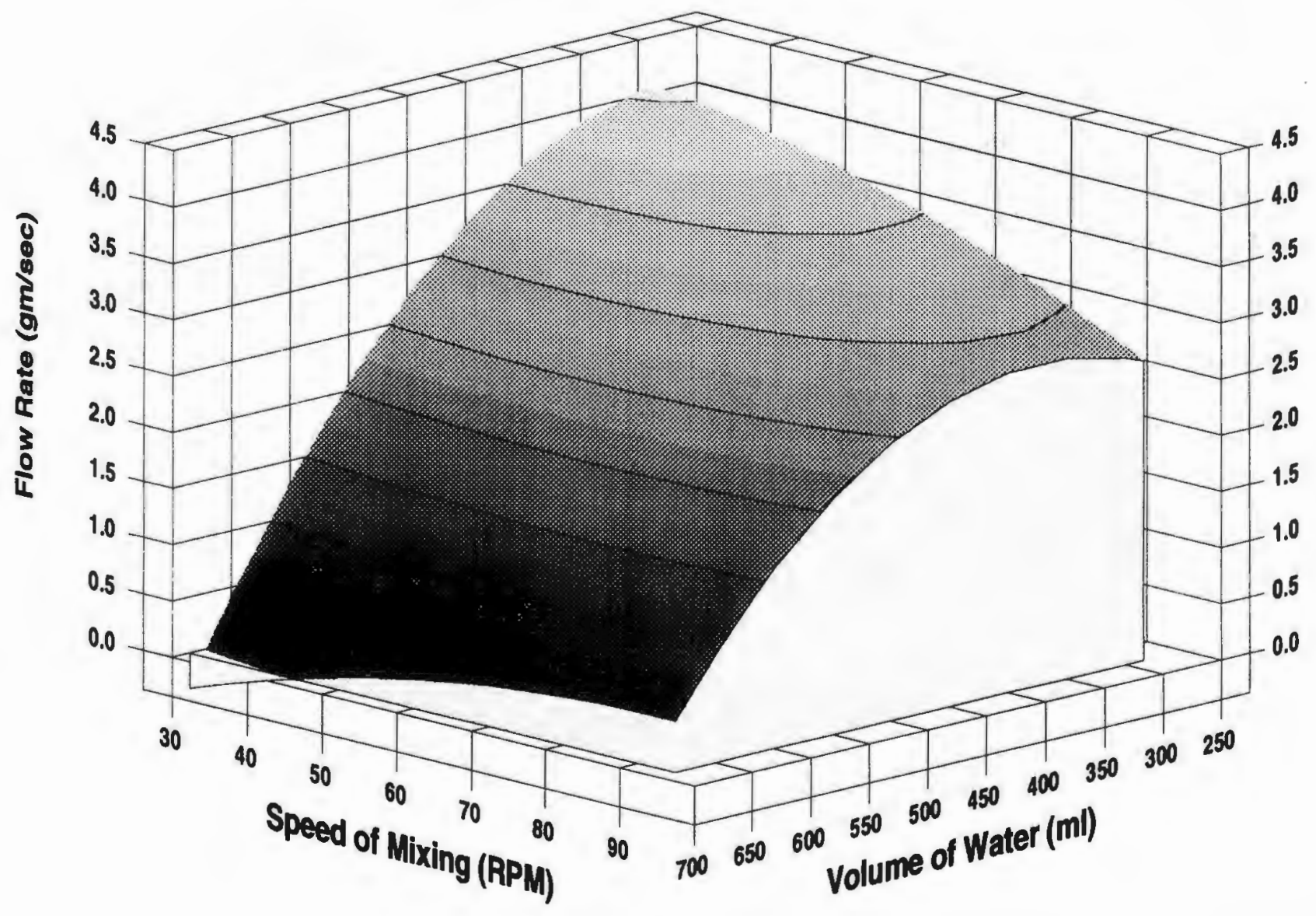




\section{Dosage Uniformity}

Direct tests of dosage uniformity are critical measures of pharmaceutical quality. Tablets are nearly always examined for their weight or thickness uniformities. An indirect, but highly useful measure of dosage uniformity is the variability of the compressional forces used in the tableting process. Instrumented tablet presses can measure the forces used during compaction, making it possible to identify doage units which fall outside some predetermined range. Tablets which require too much or too little force to compress will likely be of improper weight or thickness (or both). In this experiment we measure all three parameters to find the those best described by response surface methodology.

Weight uniformity is usually described using the coefficient of variation (sometimes called the relative standard deviation, RSD). Augsberger (1965) proposed its use an indicator of powder flow to examine glidant efficacy. The advantages of tablet weight and tablet thickness variabilities as measures of uniformity include ease and accuracy of the tests. The following table summarizes the statistical analysis for all three parameters. 
Table 2

Significant Factors ( $p$ values) Affecting Tablet Uniformity

\begin{tabular}{|c|c|c|c|}
\hline & $\begin{array}{c}\text { Weight } \\
\text { Uniformity (RSD) }\end{array}$ & $\begin{array}{c}\text { Thickness } \\
\text { Uniformity (RSD) }\end{array}$ & $\begin{array}{c}\text { Compression } \\
\text { Peak Force RSD }\end{array}$ \\
\hline & $r^{2}=0.13$ & $r^{2}=0.49$ & $r^{2}=0.60$ \\
\hline Speed of Mixing (S) & - & - & - \\
\hline Water Level (V) & - & - & - \\
\hline Speed•Speed (S $)$ & 0.033 & - & 0.0001 \\
\hline Water Water (V) & 0.056 & 0.0045 & - \\
\hline Speed•Water (SV) & 0.029 & - & 0.0001 \\
\hline
\end{tabular}

('-' indicates statistically not significant)

Experimental results for tablet thickness uniformity closely parallel those for compression force variability. Both surfaces fit the data about equally well (as per $r^{2}$ ). Although different terms in the second-order model are significant for each parameter, both surfaces appear quite similar (Figures 4 and 5). This suggests a close association between the two. Tablet weight uniformity, on the other hand, does not correlate well with either.

The variabilities in thickness and compression force attain their highest (worst) levels when the two main factors are at their maxima. The best (lowest) values are located in regions where less water is used, mixing speed notwithstanding. Uniformities of both parameters which represent the best $25 \%$ (or 75 th percentile) are shown in Table 3. 
Table 3

Areas of Best Tablet Uniformity (75th Percentile) vs. Water Level

\begin{tabular}{|c|c|}
\hline Tablet Thickness Variability & $\leq 445 \mathrm{ml}$ of water \\
\hline Compression Peak Force Variability & $\leq 385 \mathrm{ml}$ of water \\
\hline
\end{tabular}


Figure 4

Compression Force Variability (RSD, \%)

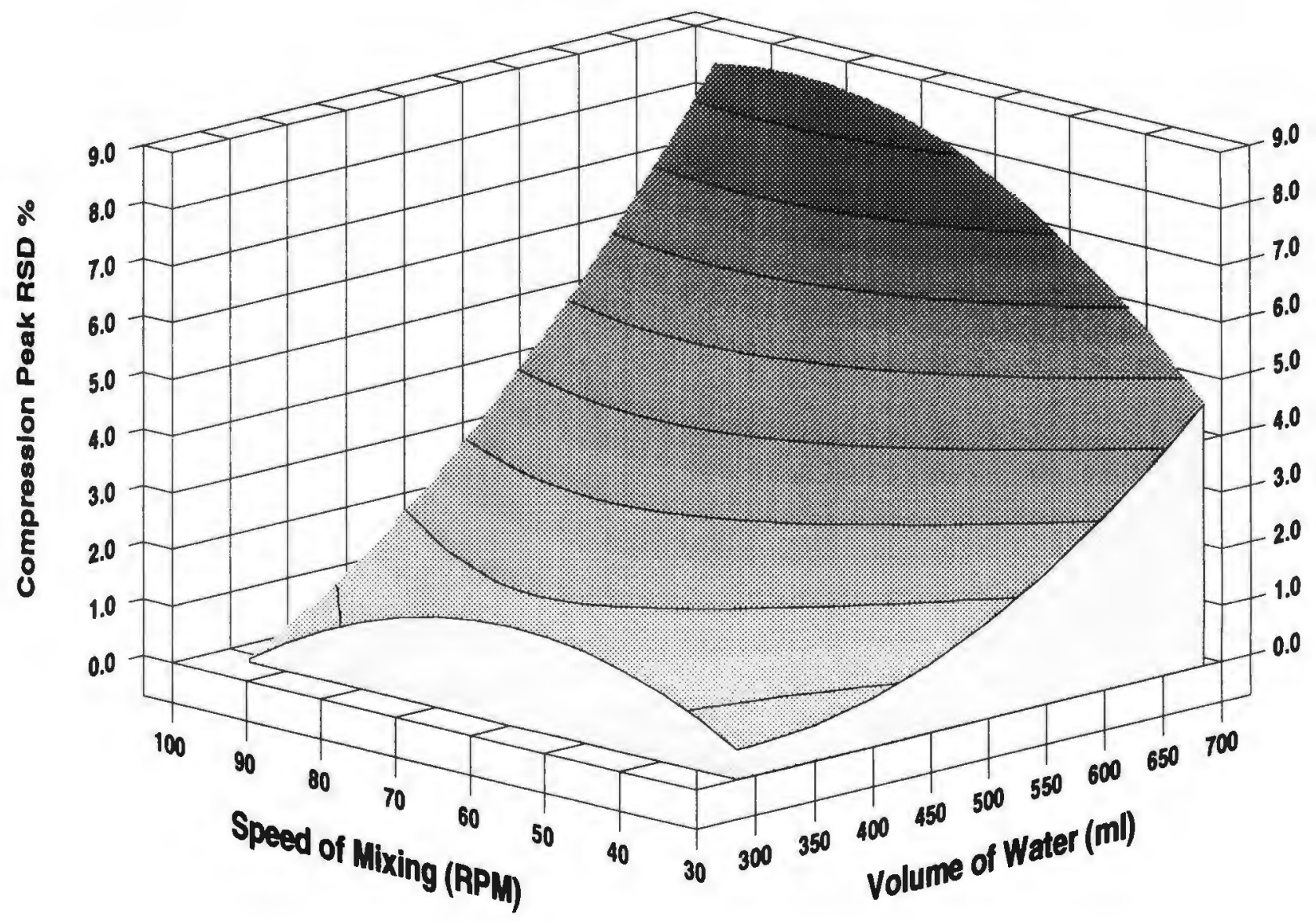


Figure 5

Tablet Thickness Uniformity (RSD,\%)

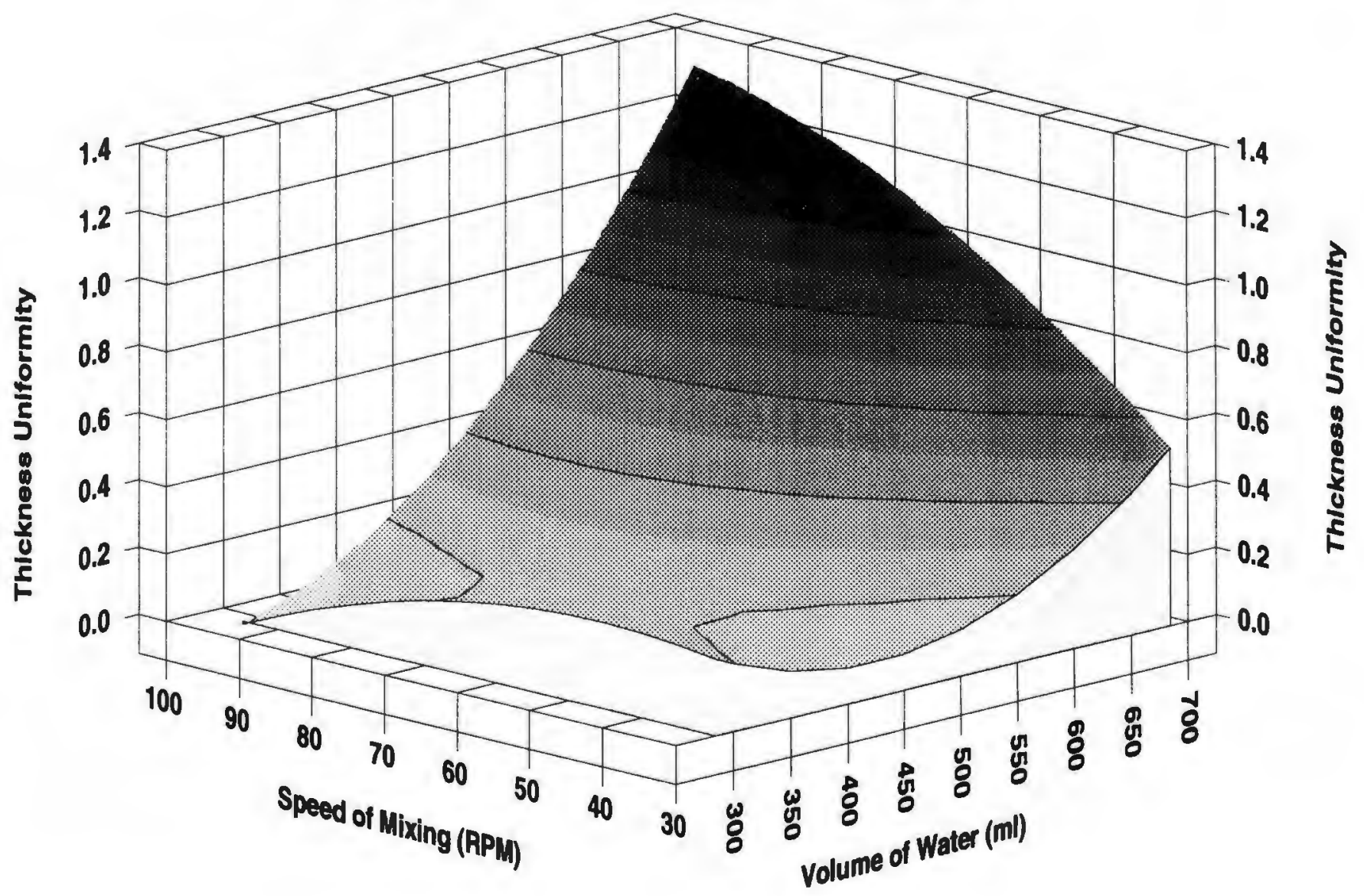


Granulation Compactability

The ability of various materials to form hard, uniform compacts is of great pharmaceutical importance. Compactability has been defined as the ability of a powdered material to be compressed into a tablet of specified strength (Chichester, 1988).

Tablets are formed when minute particles contained within die cavities are compressed by punch tools on a single station or rotary tablet press. Once formed, the compact is pushed out of the die by the lower tablet punch. In this study, we use the measurement of compression and ejection forces to gauge the effects of processing factors on the quality of the granulations and their resulting compactabilities.

Several different approaches are used to quantify various aspects of the tableting process. The use of force-time and force-displacement profiles, Heckel plots and other theoretical and empirical relationships have been developed to characterize the strength of materials, describe the deformation process, and quantify changes in density or porosity as functions of applied force. These have been extensively reviewed by Celik (1992). This experiment uses force-time data to profile the compression-ejection event. Maximal instantaneous (peak) forces and integrated areas under the force-time curves are the output variables.

Veesler (1992) uses the slope of the force-hardness profile to define a 'cohesion index'. The higher the cohesion index is, the better the compressibility. This experiment uses the cohesion index to parameterize compactability. It has a convenient feature in that neither the compression force nor the tablet hardness need to be as precisely controlled (some variability is inevitable), since it is the ratio of the two which is the response variable. Nevertheless, every effort is made to do so.

The result of the regression analysis shows mixing speed has almost no effect on compactability. Compactability is, instead, related to the amount of water used. 


\section{$C I=2.2+0.007 \cdot S-0.005 \cdot V+0.00003 \cdot S^{2}-0.00002 \cdot S \cdot V+0.000006 \cdot V^{2}$}

Significant Factors ( $p$ values) Affecting the Cohesion Index (CI)

\begin{tabular}{|c|c|c|c|c|c|}
\hline & Speed & Volume & Speed $^{2}$ & Speed Volume & Volume $^{2}$ \\
\hline CI & - & 0.0020 & - & - & 0.0021 \\
\hline
\end{tabular}

Figure 6 shows compactability is enhanced when moderate amounts of water are used, and worsens symmetrically when too much or too little is used. Minimum (best) results are obtained when approximately $435 \mathrm{ml}$ of water are used in the process, although excellent results can be obtained over a wide range $(360-500 \mathrm{ml})$ at almost any mixing speed. 
Figure 6

Tablet Cohesion Index (kg/kN)

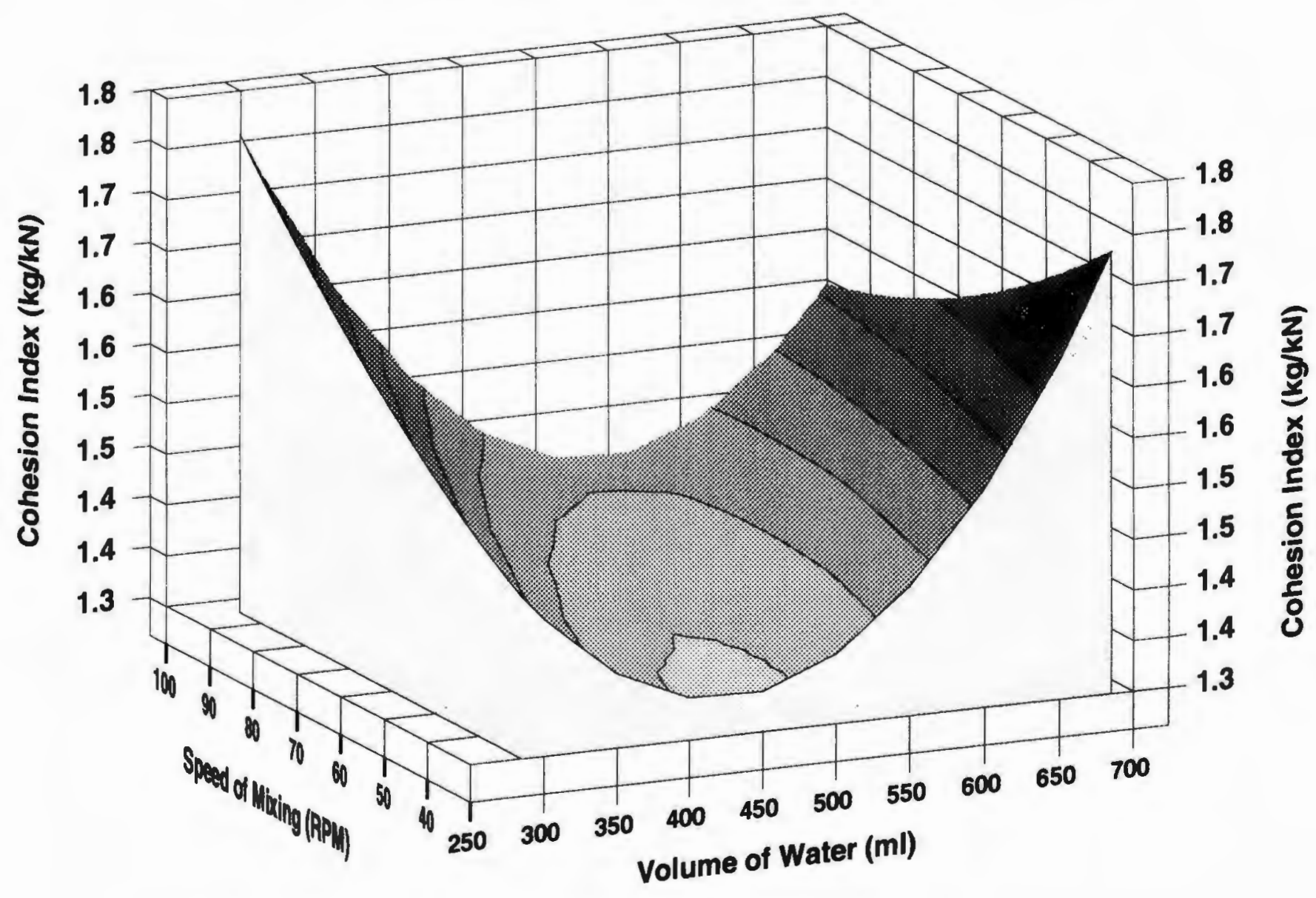


Ejection Force Requirements

Hard compacts generally require more force to be ejected from tablet dies than soft ones. In fact, data from this experiment reveals that the ejection force requirement is highly sensitive to changes in tablet strength. This is due to friction caused by residual pressures exerted on the die walls following the compression event. Part of the axial force imparted by the descending upper punch onto the powder bed is transmitted radially and maintained as long as the tablet remains there, assuming the tablet itself can withstand these radial forces without rupture. Hard tablets, then, generally exert higher die wall pressures than softer compacts.

Generally, we wish to produce hard, durable tablets and yet minimize the ejectional force requirement. This experiment measures and compares ejectional forces for each granulation. To normalize the effects of small variations in tablet hardness on individual ejection profiles, the following Ejection Force Index (EFI) is used as the output variable:

$$
\text { Ejection Force Index }(E F I)=\frac{\text { Ejection Force }(N)}{\text { Tablet Hardness }(k g)}
$$

Response surface methods show the model to be significant $(p=0.0009)$, with a reasonably good fit to the data $\left(r^{2}=0.77\right)$. Backward elimination procedures reveal the VOLUME OF WATER to be the sole independent variable of significance.

Ejection force is minimized where both factor are at their highest levels (Figure 7), resulting in ejection forces that are approximately $60 \%$ of maximum. Maximal ejection 
forces are required for granulations made with minimum amounts of water, regardless of mixing speed.

In the absence of lubricant, the amount of ejection force required is governed by residual die wall pressures and friction factors. Following lubrication, this information is complicated by the effects of the added agent. The amount of lubricant required to satisfactorily lower ejectional forces depends upon the amount of exposed granulation surface area which, in turn, depends upon the mean particle size and size distribution. Larger particles have less surface area on a weight basis than smaller particles. The same amount of lubricant added to a unit weight of larger particles 'sees' less surface and covers it more thoroughly.

A relative measure of particle surface area can be obtained using the volumesurface mean diameter $d_{v s}$. An increase in $d_{v s}$ indicates a proportional decrease in surface area. Response surfaces for $d_{v s}$ and the ejection force index (Figures 8 and 7) show that ejection force reaches a minimum in the same region where particle sizes are greatest (or where surface area is smallest). Similarly, highest levels of ejection force occur where minimal amounts of water are used, which coincides with the smallest particle sizes. Both parameters increase proportionally to the square of the amount of water used, and neither is a strong function of mixing speed. These results suggest that ejection force is inversely related to particle size and is proportional to changes in surface area. 


\section{A Comparison of}

Ejection Force $\div$ Tablet Hardness vs. Volume-Surface Mean Diameter

Response Surface Regression Analysis

$$
\begin{aligned}
E F I & =248.2+0.71 \cdot S-0.52 \cdot V+0.0037 \cdot S^{2}-0.0028 \cdot S \cdot V+0.00061 \cdot V^{2} \\
d_{v s} & =273.0+8.95 \cdot S-0.058 \cdot V+0.0031 \cdot S^{2}-0.0144 \cdot S \cdot V+0.0023 \cdot V^{2}
\end{aligned}
$$

Significant Factors ( $p$ values) Affecting the Ejection Force Index

\begin{tabular}{|c|c|c|c|c|c|}
\hline & Speed & Volume & Speed $^{2}$ & Speed Volume & Volume $^{2}$ \\
\hline EFI & - & 0.008 & - & - & 0.0045 \\
\hline
\end{tabular}


Figure 7

Tablet Ejection Force Index (N/kg)

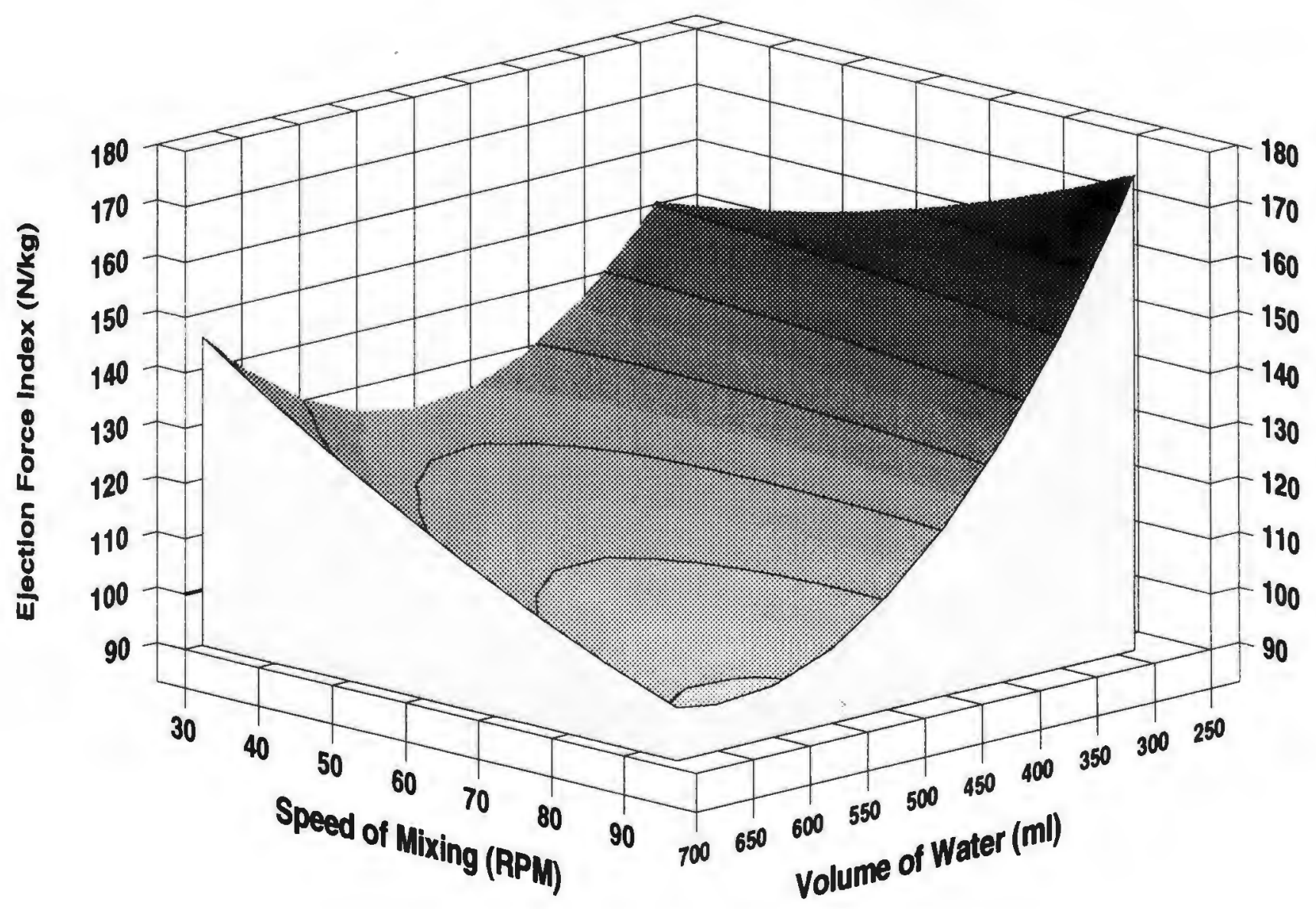


Figure 8

\section{Surface-Volume Mean Particle Size (microns)}

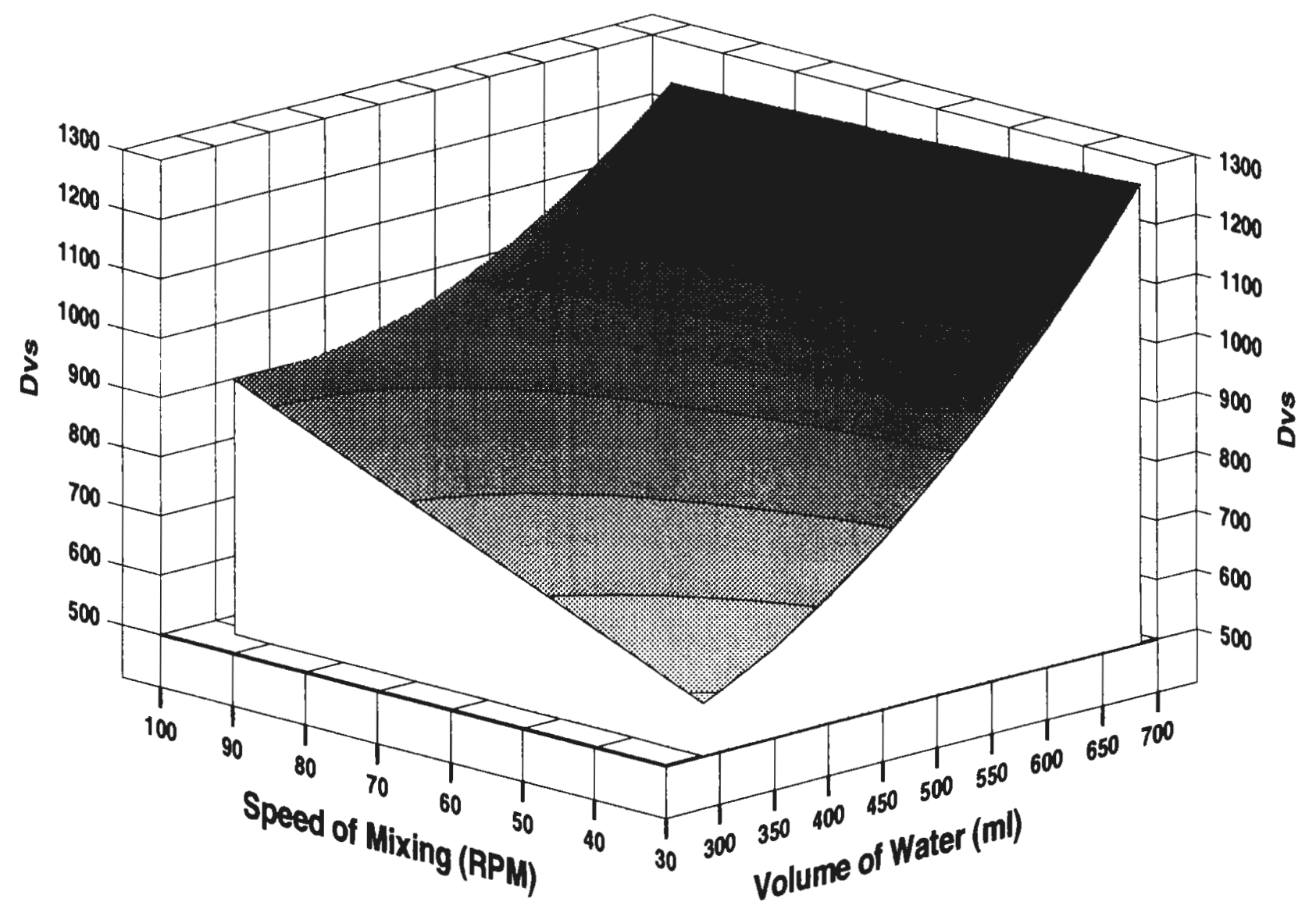


Hardness-Force Index

The cohesion index is an indicator of compactability. The ejection force index provides a relative measure of lubricity, residual die wall stresses and friction coefficients. These parameters are generally used independently of each other to examine different aspects of the tableting process. Ultimately, however, it would useful to determine which granulations are both easiest to compress and easiest to eject. In other words, those granulations which have the best ratios of compression to ejection force may be the most tabletable. We can therefore combine both ratios into a single parameter, the hardnessforce index. Its derivation is as follows:

$$
\begin{aligned}
\text { Hardness }- \text { Force Index } & =\frac{\text { Hardness } \div \text { Compression Force AUC }}{\text { Ejection Force AUC } \div \text { Hardness }} \\
& =\frac{\text { Hardness }^{2}}{\text { Compression Force AUC } \times \text { Ejection Force AUC }}
\end{aligned}
$$

Since increases in compression force are reflected in concommitant increases in tablet hardness for compressible materials, and because these same increases in hardness make it more difficult to eject tablets from their dies, it is not clear a priori which effect will predominate. Similarly, lubricants reduce the ejection force requirement, but may also reduce tablet hardness. Once again, it is difficult to predict the ultimate effect on the tableting process. The hardness-force index (HFI) combines all of the above considerations into a single parameter. A regression analysis using the hardness-force parameter shows the following results: 
Re sponse Surface Regression Analysis

$H F I\left(\times 10^{3}\right)=-582-8.34 \cdot S+10.1 \cdot V-0.17 \cdot S^{2}+0.065 \cdot S \cdot V-0.013 \bullet V^{2}$

Significant Factors ( $p$ values) Affecting the Hardness-force Index (HFI)

\begin{tabular}{|c|c|c|c|c|c|}
\hline & Speed & Volume & Speed $^{2}$ & Speed•Volume & Volume $^{2}$ \\
\hline HFI & - & 0.0081 & - & - & 0.0165 \\
\hline
\end{tabular}

Response surface methodology shows the regression to be significant $(p=0.007)$ with a moderate $r^{2}=0.56$. Results show that maximum values of the HFI are located in regions where fairly high $(570 \mathrm{ml})$ levels of water are used (Figure 9). Satisfactory (best $20 \%$ ) results can be attained using moderate amounts of water at most mixing speeds. In all, the HFI tends to reflect decreases in ejection force for this granulation, moreso than increases in compactability. This can be explained by the good compressibility of the starting materials, which is not the case for many pharmaceutical materials. 
Figure 9

Tablet Hardness-Force Index (HFI)

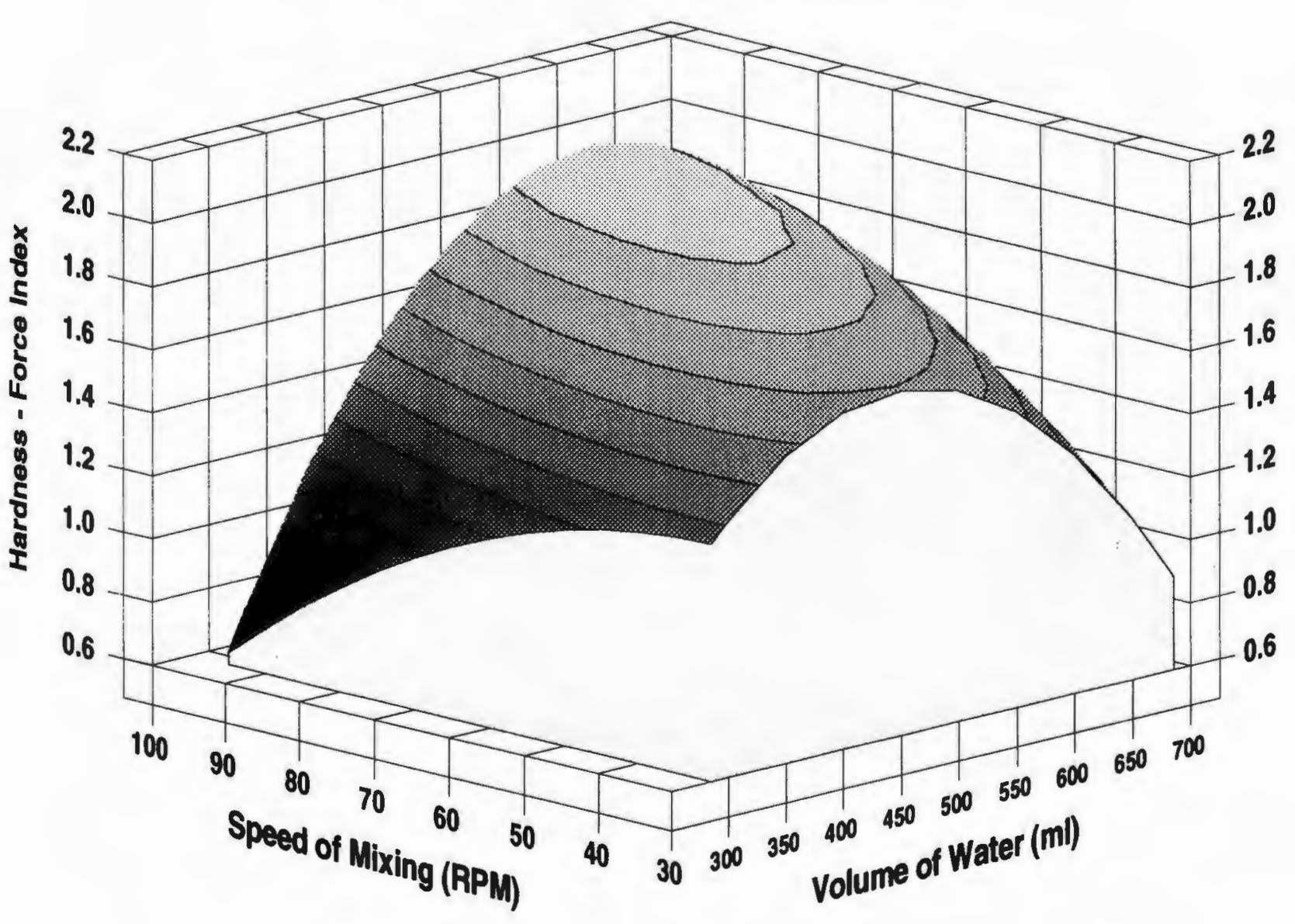


Disintegration Time (DT)

Response surface methodology yields the following summary of results:

Response Surface Regression Analysis

$D T=7.80-0.09 \cdot S-0.01 \cdot V+0.0007 \cdot S^{2}+0.00003 \cdot S \cdot V+0.00002 \cdot V^{2}$

Significant Factors ( $p$ values) Affecting the Disintegration Time

\begin{tabular}{|c|c|c|c|c|c|}
\hline & Speed & Volume & Speed $^{2}$ & Speed Volume & Volume $^{2}$ \\
\hline Disintegration & - & - & - & - & 0.049 \\
\hline
\end{tabular}

Overall, the regression is only marginally significant $(p=0.0485)$ and suffers from a low $r^{2}$. Minimal values for disintegration time, the desired result, occur where low to moderate amounts of water $(285-435 \mathrm{ml})$ are used with medium mixing speeds (Figure 10). These results suggest that disintegration times are likely to be relatively short where the granules themselves are smaller prior to compression. 
Figure 10

Tablet Disintegration Time (min)

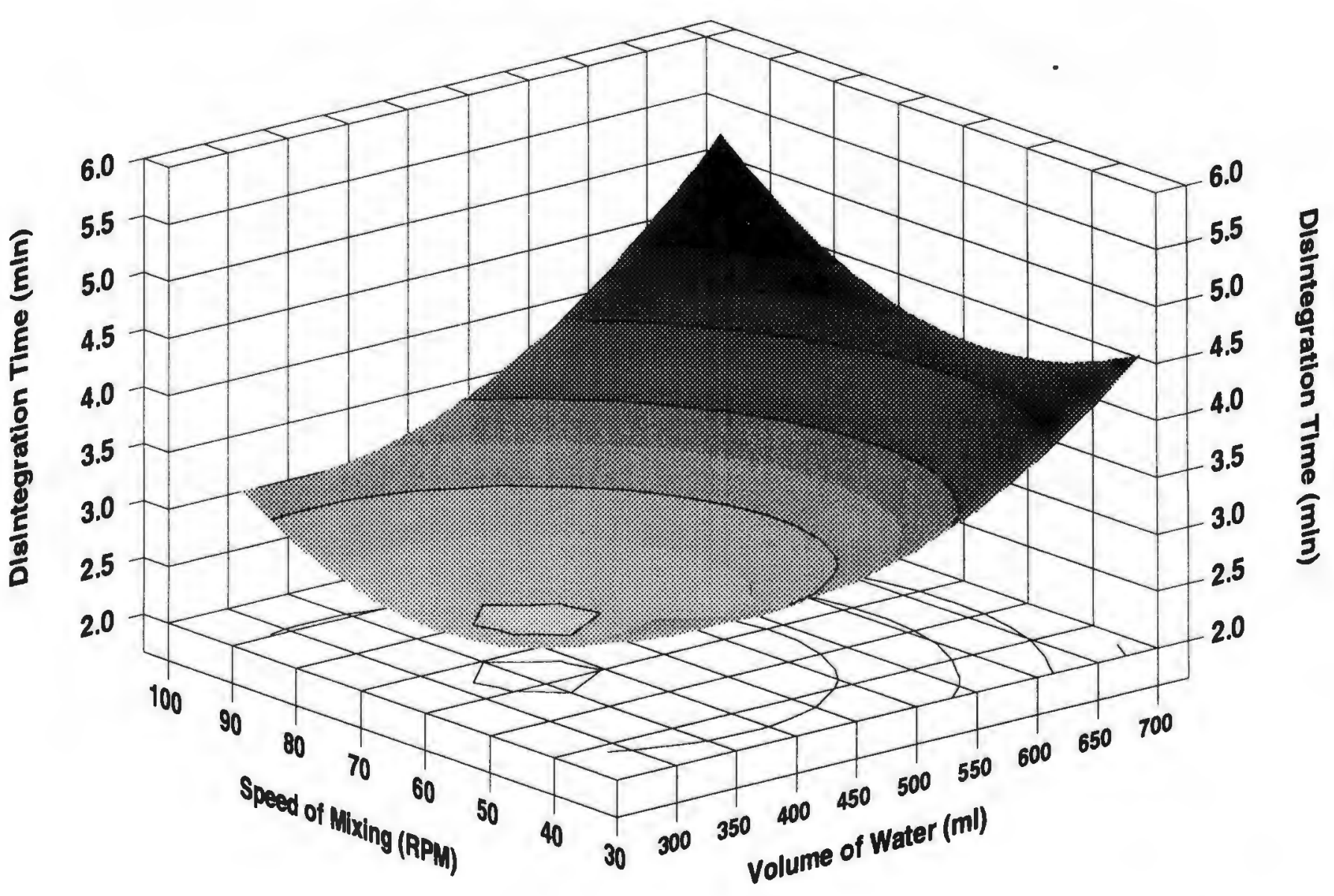




\section{Optimization}

The optimization search is carried out using linear programming with SASß software to perform a systematic incremental grid search procedure of all possible values of the two independent variables which satisfy all constraints. The following key parameters have been selected for inclusion in the optimization search, along with the imposed constraints on each:

Table 4

Optimization Constraints

\begin{tabular}{|l|c|c|}
\hline Granulation Flow Rate & $\geq 3.25$ & $\mathrm{gm} / \mathrm{sec}$ \\
\hline Granulation Particle Size Uniformity & $\leq 1.84$ & $\left(\sigma_{g e o}\right)$ \\
\hline Tablet Cohesion Index (CI) & $\leq 1.400$ & $\mathrm{~kg} / \mathrm{kN}$ \\
\hline Hardness-Force Index (HFI) & $\leq 1.7000$ & $\frac{\mathrm{kg}^{2}}{\mathrm{kN}^{2} \times \mathrm{msec}^{2}}$ \\
\hline Tablet Thickness Uniformity & $\leq 0.300$ & $\mathrm{RSD}, \%$ \\
\hline Compression Peak Force Uniformity & $\leq 3.000$ & $\mathrm{RSD}, \%$ \\
\hline Tablet Disintegration Time & $\leq 3.077$ & $\mathrm{minutes}$ \\
\hline
\end{tabular}


The following sets of points satisfy all of the above criteria:

Table 5

Optimum Factor Levels

\begin{tabular}{|c|c|c|c|c|c|}
\hline Mixing Speed (rpm) & \multicolumn{4}{|c|}{ Water Levels (ml) } & \\
\hline 45 & 385 & & & & \\
\hline 50 & 385 & 395 & & & \\
\hline 55 & & 395 & 405 & 415 & \\
\hline 60 & & 395 & 405 & 415 & 425 \\
\hline 65 & & & 415 & & \\
\hline
\end{tabular}

Further tightening of constraints, such as increasing the minimum acceptable value of the hardness-force index (HFI) to 1.800 , results in a single satisfactory point at $60 \mathrm{rpm}$ and $425 \mathrm{ml}$ (bold italics). Note that no actual experiments were performed at any of these combinations of variables.

Each of these points can now be used to calculated predicted responses for response variables not used in the search. The response surface for the geometric mean particle size for example, shows the location of these eleven points (Figure 11) from which target optimal particle sizes can be determined $(578 \mu-681 \mu)$. 
Figure 11

Geometric Mean Diameter (microns) with Optimal Factor Levels Shown

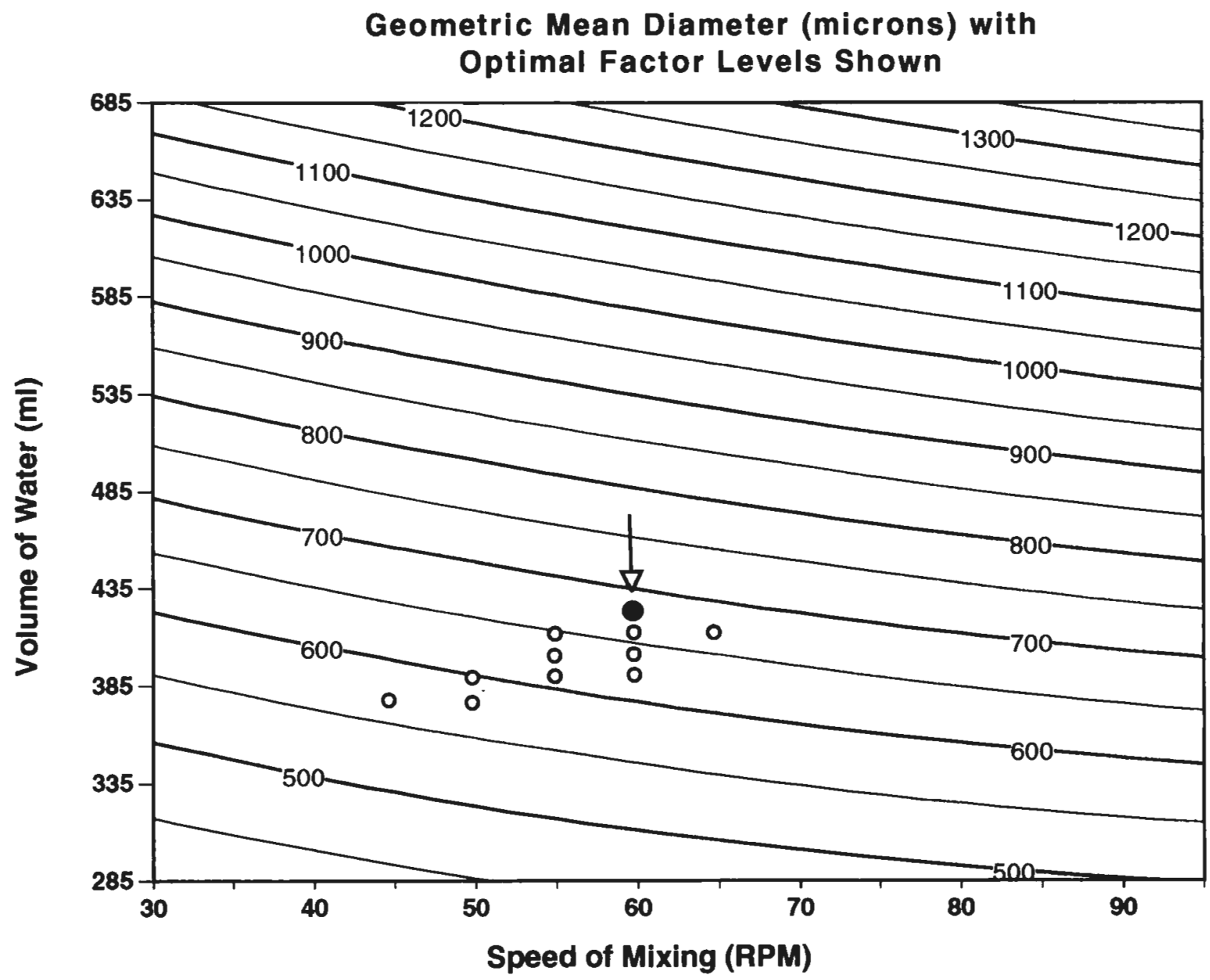


CONCLUSIONS

The Ross Double Planetary Mixer does not function as a high-shear mixer. Its speed of mixing plays only a minor role in the wet granulation process. It was unable to control particle size, as required. It functioned to distribute fluid throughout the powder mass and cause agglomeration, but it could not then reduce particle size through shear.

The mixer was able to generate enough speed, however, to modify the effects of water addition on several variables. Particle size, size uniformity, and compression force uniformity were altered when mixing speed was varied. These effects manifested themselves statistically as interactions between mixer and speed.

Only one variable, the compressional force variability, was quite sensitive to mixing speed, but its effect could not be supported by similar effects on tablet weight or thickness variability. In any case, the effect of mixing speed on compression force variability was to increase it, not to decrease it. This serves to fortify our contention that the mixer's speed is inadequate to qualify it as a high-shear design.

The formulation used in this study, dibasic calcium phosphate and lactose and additional binder, required very little granulation to enhance its properties. Optimization procedures located a combination of variables which yielded points satisfying seven different response variables with constraints. These eleven points represented mixing speeds of 45 to $65 \mathrm{rpm}$ and water levels of 385 to $425 \mathrm{ml}$. A further tightening of constraints singled out a single point $(60 \mathrm{rpm}, 425 \mathrm{ml})$. It is worth noting here that the center point of the central composite design was chosen by the experimenter to be close to a suspected optimum. This combination ( $67 \mathrm{rpm}$ and $485 \mathrm{ml}$ of water), is indeed fairly close, but suggests that the formulation may actually require less aggressive wet granulation than first thought. 
This study proposes the use of a new parameter, the Hardness-Force Index (HFI) as a gauge of tabletability. It combines the essentials of both compactability and ejectability into one response. Further work is necessary, however, to determine whether this new parameter has any usefulness for characterizing a pharmaceutical tableting process. 


\section{REFERENCES}

Augsburger L.L., Shangraw R.F. (1966) Effect of Glidants in Tableting, Journal of Pharmaceutical Sciences, 55(4), 418-423.

Bohidar N.R. (1991) Pharmaceutical Formulation Optimization Using SAS ${ }^{\mathrm{TM}}$, Drug Development and Industrial Pharmacy, 17(3), 421-441.

Box G.E.P. (1954) The Exploration and Exploitation of Response Surfaces: Some General Considerations and Examples, 10, 16-60.

Box G.E.P., Draper N. (1987) Empirical Model Building and Response Surfaces, John Wiley \& Sons, New York, p. 511.

Carr R.L. (1965) Evaluating Flow Properties of Solids, Chemical Engineering, 72, 163168.

Celik M. (1992) Overview of compaction Data Analysis Techniques, Drug Development and Industrial Pharmacy, 18(6\&7), 767-810.

Chichester E.H. (1988) Tablet Machine Instrumentation in Pharmaceutics: Principles and Practice, Halsted Press, New York.

Cliff M.J. (1990) Granulation End Point and Automated Process of Mixer-Granulators:Part I, Pharmaceutical Technology, 14(4),112-132. 
Cliff M.J. (1990) Granulation End Point and Automated Process of Mixer-Granulators:Part II, Pharmaceutical Technology, 14(5),36-44.

Corvari V., Fry W.C., Seibert W.L., Augsberger L. (1992) Instrumentation of a HighShear Mixer: Evaluation and Comparison of a New Capacitive Sensor, a Watt Meter, and a Strain-Gauge Torque Sensor for Wet Granulation Monitoring, Pharmaceutical Research, 9(12), 1525-1533.

Harris M.R., Schwartz J.B., McGinity J.W. (1985) Optimization of a Slow-Release Tablet Formulation Containing Sodium Sulfathiazole and Montmorillonite Clay, Drug Development and Industrial Pharmacy, 11(5), 1089-1110.

Kristensen H.G., Schaefer T. (1987) A Review of Pharmaceutical Wet-Granulation, Drug Development and Industrial Pharmacy, 13(4\&5), 812-813.

Lachman L., Lieberman H.A., Kanig J.L. (1986) The Theory and Practice of Industrial Pharmacy, Third Edition, Lea \& Febiger, Philadelphia.

Newitt D.M., Conway-Jones J.M. (1958) Transactions of the Institution of Chemical Engineers, 36, 422.

Parrott E.L. (1970) Pharmaceutical Technology, Burgess Publishing Company, Minneapolis. 
Pendharkar C.M.,Jhawar R.J., Rutledge J.M., Hause W., Grim W.M., Harwood R.J. (1990) Influence of the Specific Surface Area of Selected Raw Materials on the Granulation Process Using an Instrumented Mixer, Pharmaceutical Technology, 14(4), 44-53.

Schwartz J.B. (1981) Optimization Techniques in Product Formulation, Journal of the Society of Cosmetic Chemists, 32,287-301.

Schwartz J.B. (1988) Granulation, Drug Development and Industrial Pharmacy, 14(14), 2071-2090.

Schwartz J.B., Flamholz J.R., Press R.H. (1973) Computer Optimization of Pharmaceutical Formulations I:General Procedure, Journal of Pharmaceutical Sciences, 62(7), 1165-1170.

Schwartz J.B., Flamholz J.R., Press R.H. (1973) Computer Optimization of Pharmaceutical Formulations II:Application in Troubleshooting, Journal of Pharmaceutical Sciences, 62(7),1165-1170.

Timko R.J., Barrett J.S., McHugh P.A., Chen S.T., Rosenberg H.A. (1987) Use of a Motor Load Analyzer to Monitor the Granulation Process in a High Intensity Mixer, Drug Development and Industrial Pharmacy, 13(3), 403-435.

Usteri M., Leuenberger H. (1989) Agglomeration of Binary Mixtures in a High-Speed Mixer, International Journal of Pharmaceutics, 55, 135-141. 
Veesler S., Boistelle R., Delacourte A., Guyot J.C., Guyot-Hermann A.M. (1992) Influence of Structure and Size of Crystalline Aggregates on Their Compression Ability, Drug Development and Industrial Pharmacy, 18(5), 539-560.

Vojnovic D., Selenati P., Rubessa F., Moneghini M., Zanchetta A. (1992) Wet Granulation in a Small Scale High Shear, Drug Development and Industrial Pharmacy, 18(9), 961-972.

Vojnovic D., Rubessa F., Moneghini M., Zanchetta A. (1993) Simultaneous Optimization of Several Response Variables in a Granulation Process, Drug Development and Industrial Pharmacy, 19(12), 1479-1496.

Vojnovic D., Moneghini M., Rubessa F. (1994) Optimization of Granulates in a High Shear Mixer by Mixer Design, Drug Development and Industrial Pharmacy, 20(6), 10351047. 
Manuscript II

A Comparative Evaluation of Two Pregelatinized Starches and Two High-Speed Mixer Designs

in the Development of a Compressible Acetaminophen Product 
Acetaminophen is a widely used drug substance which usually requires some type of size enlargement process before it can be formed into tablets. This study examines three variables in an effort to develop a successful directly-compressible acetaminophen-starch product. Two high-shear mixers of different configurations, two pregelatinized starches and two levels of mixing speed are combined into a complete factorial experiment designed to identify and quantify the importance of these factors and any potential interactions between them.

Ten response variables relating to granulations, tablets and tableting process parameters are used as output variables. Results show that mixing speed is an important variable affecting nearly every response variable, usually causing a direct improvement. The effects of mixing speed depended somewhat on which mixer was used. The PowerMix generally outperformed the Fielder, leading to the conclusion that the Powermix has a more intense mixing action. There were impotant differences between starches. Starch 1500 made stronger compacts, as measured by compactability and durability. Its granulations had significantly better powder flow as well. 
INTRODUCTION

One of the most common drugs in use today is acetaminophen, a non-prescription pain reliever and antipyretic. It is most often administered as a tablet, but acetaminophen in its pure form is poorly suited for many pharmaceutical unit operations. Firstly, its relatively low potency as a drug requires that relatively large amounts must be used (e.g. $500 \mathrm{mg}$ per tablet). Secondly, its cohesive and adhesive nature leads to poor powder flow characteristics. Thirdly, it is poorly compressible, forming tablets that are generally soft and not very durable.

Attempts have been made over the years to develop a freely-flowing, compressible acetaminophen powder containing approximately $90 \%$ drug and $10 \%$ excipient. Some commercial processes utilize fluid-bed granulation techniques (Salpekar and Denton (1987), Vogel (1984)). Patel, et al. (1989) and Liu, et al. (1994) used fluid-bed granulation techniques using additional binding agents to make a directly-compressible acetaminophen powder. More commonly, however, wet granulation is carried out in either slow or high-speed mixer-processors. This investigation attempts to manufacture a compressible acetaminophen-starch granulation using traditional wet granulation methods.

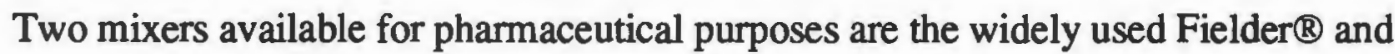
the Ross PowerMix@. Both are of the high-speed variety, but differ significantly in design configuration. The PowerMix claims to have the advantage of a mobile, orbiting highspeed chopper. The Fielder has its chopper fixed in position on the interior wall of the bowl. The mobility of the mixing tools could serve to reach less accessible regions within the mixing space, rather than relying upon the impeller arm of the Fielder to help move material past the wall-mounted mixing tool. No studies exist to date which directly compare these two designs in terms of their utility for pharmaceutical purposes. 
Starch is a very commonly used polysaccharide diluent. It is useful due to its relative inertness, self-lubricating qualities, disintegrant activity and low cost (Swarbrick, 1988). Efforts to improve its binding and flow properties have lead to the introduction of modified starches. These pregelatinized starches are prepared from ordinary corn starch by heating under high moisture conditions. Though marketed primarily as direct compression excipients, Schwartz (1981) used it successfully in wet granulation processes. Two such starches are available, Starch 1500 (Colorcon, Inc.) and Starch 1551 (National Starch). Starch 1500 is partially pregelatinized, while Starch 1551 is fully pregelatinized. Comparative evaluations of the performance of these two starches is lacking, although anecdotal evidence seems to favor Starch 1551.

A factorial design experiment was conducted to test the following differences:

1) For the wet granulation of acetaminophen-pregelatinized starch combinations, is one mixer superior to the other?

2) In the manufacture of acetaminophen-pregelatinized starch combinations using high-speed processing equipment, is there a difference between the two commercial grades of modified starch?

3) Is mixing speed an important variable affecting formulation performance? 
EXPERIMENTAL

Materials

Starch 1500 (Colorcon, Inc., West Point, PA) and Starch 1551 (National Starch Lot\# FJ7343, Bridgewater, NJ) were obtained from Colorcon, Inc. Acetaminophen USP was obtained from Mallinckrodt (Lot\#4899-2K-768). Magnesium Stearate NF was obtained from Fisher Scientific (Lot\#742748, Fairlawn, NJ).

Formula

\begin{tabular}{|l|c|c|}
\hline Ingredient & $\mathrm{mg} / \mathrm{tab}$ & $\%$ \\
\hline Acetaminophen USP & 337.500 & 90.00 \\
\hline Pregelatinized Starch NF & 35.625 & 9.50 \\
\hline Purified Water USP & $*$ & $*$ \\
\hline Magnesium Stearate NF & 1.875 & 0.50 \\
\hline Total & 375.000 & 100.00 \\
\hline
\end{tabular}

* Water is expelled during processing 
METHODS

Experimental Design

The experimental design for this experiment is a three-factor two-level full factorial design with one replicate (Figure 1). Statistical analyses were performed using SAS ${ }^{\mathrm{TM}}$ (SAS Institute, Cary, NC, Release 6.08). Backward elimination regression analyses use 0.10 as the criteria for retention in the model (SLSTAY $=0.10$ ).

Granulation

The Ross PowerMix ${ }^{\circledR}$ Model PD-2 was made available from Charles Ross \& Son Co., Inc. Hauppauge, NY. Working capacity 1.5 gallons; planetary stirrer at $19 \mathrm{~Hz}$; highspeed disperser $19 \mathrm{~Hz}$ (low speed) and $55 \mathrm{~Hz}$ (high speed). The Fielder 2-gallon capacity was made available at Colorcon Inc. (Niro-Aeromatic, Columbia, MD).

Liquid was added during granulation by pumping at $60 \mathrm{ml} / \mathrm{min}$ using a Masterflex Peristaltic Pump \#7553-30. Drying was done in a circulating hot air oven (Thelco Tray Oven Model 28).

Milling operations were performed using a Stokes Oscillating Mill through a \#12 wire mesh screen. Lubrication with Magnesium Stearate was performed in a Turbula Blender. 
Figure 1

\section{Experimental Design}

Three-Factor, Two-Level Full Factorial

\begin{tabular}{|c|c|c|c|c|c|}
\hline Processor & Chopper Speed & Starch & \multicolumn{3}{|c|}{ Factor Levels } \\
\hline Fielder & Low & 1500 & - & - & - \\
\hline PowerMix & Low & 1500 & + & - & - \\
\hline Fielder & High & 1500 & - & + & - \\
\hline PowerMix & High & 1500 & + & + & - \\
\hline Fielder & Low & 1551 & - & - & + \\
\hline PowerMix & Low & 1551 & + & - & + \\
\hline Fielder & High & 1551 & - & + & + \\
\hline PowerMix & High & 1551 & + & + & + \\
\hline
\end{tabular}




\section{Particle Size Testing}

Sieve analyses were performed with approximately 20 -gram samples of dried granulate added to a stack of US Standard Sieves, dried and tared. Sieving was done using a Van-Kel Model 18480 Sieve Shaker for 5 minutes at a \#7 setting, or until no further change takes place. Analyses are performed in triplicate. The geometric mean, volumesurface mean sizes and geometric standard deviation are calculated directly using the formulas given in Manuscript I (see Experimental Section).

\section{Bulk Density}

Free-flowing and tapped densities were determined by pouring $100 \mathrm{ml}$ samples through a funnel into a graduated cylinder, then tapped 1000 times. Volume determinations before and after tapping ( $v$ and $v_{0}$, respectively) a known weight of granulate are used to calculate the Carr Compressibility Index (Carr, 1965), expressed as a percentage:

$$
\text { Compressibility Index }=\left[1-\frac{v}{v_{0}}\right] \cdot 100
$$

Angle of Repose

Dried unlubricated granules are poured through a funnel to a known height. The diameter of the cone-shaped pile is measured. The angle is the degrees of elevation from the horizontal, as determined by the following formula: 


$$
\text { Angle of Repose }=\arctan \left(\frac{\text { height }}{\text { radius }}\right)
$$

Tableting

Tablets were compressed using an instrumented rotary tablet press (PennwaltStokes Model 900-512) operating at $30 \mathrm{rpm}( \pm 1)$ adjusted to three compression settings approximating $12 \mathrm{kN}, 16 \mathrm{kN}$ and $20 \mathrm{kN}$. The tablets are compressed using a single punch and die set . (7/16-inch round, flat-faced, no bevel-edge) to a weight of $375 \mathrm{mg}$. During tableting, a minimum of thirty tablets are compressed. Each compression-ejection cycle is measured and recorded individually; means and standard deviations of each of the following parameters are generated by the data acquisition and analysis software:

1) Peak Compression Force

$(\mathrm{kN})$

2) Area Under Compression Force-Time Curve

(kN॰msec)

3) Peak Ejection Force

(N)

4) Area Under Ejection Force-Time Curve

(N॰msec)

The tablet 'cohesion index' is calculated from the following formula:

$$
\text { Cohesion Index }=\frac{\text { Crushing Force }(\mathrm{kg})}{\text { Peak Compression Force }(\mathrm{kN})} \times 10^{5}
$$




\section{Tablet Weight}

At least ten tablets are weighed indiviually on a Mettler AE 240 Digital Balance to an accuracy of \pm 0.00001 grams. The mean, standard deviation and relative standard deviations are determined.

\section{Tablet Thickness}

The thickness of at least ten individual tablets is measured using an analog micrometer (Mitutoyo Analog Micrometer) accurate to \pm 0.0001 inches.

\section{Tablet Hardness}

The diametral crushing strength of at least ten individual tablets is measured using the Erweka Tablet Hardness Tester Model TBT. Results are reported in kilograms.

\section{Tablet Friability}

Twenty tablets of known weight are allowed to fall from a height of six inches inside a baffled chamber revolving at 25 RPM for four minutes. The tablets are dedusted and reweighed. Friability is the loss in weight, expressed as a percentage.

Tablet Disintegration Time

The disintegration time of six individual tablets is measured using the USP Disintegration Apparatus, with water at $37^{\circ} \mathrm{C}$ as the medium. 
RESULTS AND DISCUSSION

Analysis of Raw Materials

Particle Size

Sieve analyses were performed in triplicate on three lots of Starch 1500 and one lot of Starch 1551. A sieve range of 338 microns and below (US Standard Sieve Sizes No.40 to No. 400) was used. The median particle size was determined graphically as illsutrated in Parrott (1970). The geometric mean size, geometric standard deviation and volume-surface mean sizes were calculated using formulas described in Manuscript I. Duncan's Multiple Range Test (Hines and Montgomery, 1990) for comparing all pairs of means was used to evaluate the differences between means of all four starches tested.

The median particle sizes for the four starches tested meet specifications for pregelatinized starches and show generally good agreement with published results (Boylan, et all. 1986) and The results of Duncan's Test, shown in Table 1, reveal the similarity between Starch 1551 and three lots of Starch 1500. Starch 1551 was not significantly different from Lot $\mathrm{C}$ in one grouping (means with the same letter are not significantly different)), or from lots A and B in another grouping. Moreover, there were greater differences between different lots of Starch 1500 (A \& B vs. C) than existed between the different manufacturers. Overall, the differences between all starches appear quite small and seem unliklely to have any practical significance.

An analysis of geometric mean data, shown in Table 2 supports the similarity of 1551 and 1500 Lot C, as well as the dissimilarity between starch 1500 lots A, B and C. Again, even the largest differences between starches do not appear likely to cause differences during granulation and processing. 
Table 3, the volume-surface mean for Starch 1551 was significantly smaller than for 1500 . Even Lot $C$, which did not differ from 1551 in its median and geometric mean sizes, had a dvs $19 \%$ larger than 1551 , which was significant. The volume-surface mean diameter particularly emphasizes the contribution of larger particle sizes. The differences between the starches in the particle sizes 80-mesh and larger are exaggerated, as compared with the smaller sizes. The significant difference between 1551 and 1500 correlates to a concommitant difference in their surface areas, with 1551 having the greater amount per unit volume. Determining whether this fact has any practical significance in terms of excipient performance is one of the objectives of this study. 
Table 1

Duncan's Multiple Range Test for Median Particles Sizes of Starches 1500 and 1551

\begin{tabular}{|l|c|c|c|c|}
\hline Starch Type & 1500 Lot C & 1551 & 1500 Lot A & 1500 Lot B \\
\hline Median Size & 77.6 & 74.7 & 72.7 & 71.7 \\
\hline Duncan Grouping & A & A & & \\
& & B & B & B \\
\hline
\end{tabular}

(means with the same letter are not significantly different)

Table 2

Duncan's Multiple Range Test for Geometric Mean Particles Sizes of Starches 1500 and 1551

\begin{tabular}{|l|c|c|c|c|}
\hline Starch Type & 1551 & 1500 Lot C & 1500 Lot A & 1500 Lot B \\
\hline Geometric Mean & 59.1 & 57.6 & 54.0 & 52.7 \\
\hline Duncan Grouping & A & A & B & B \\
\hline
\end{tabular}

Table 3

Duncan's Multiple Range Test for Volume-Surface Mean Particles Sizes of Starches 1500 and 1551

\begin{tabular}{|l|c|c|c|c|}
\hline Starch Type & 1500 Lot B & 1500 Lot A & 1500 Lot C & 1551 \\
\hline Surface-Volume Mean & 169.9 & 134.9 & 128.3 & 107.6 \\
\hline Duncan Grouping & A & B & B & C \\
\hline
\end{tabular}


Particle Size Distribution.

Log-probability graphs (not shown) reveal both starches to have particle sizes that are essentially log-normally distributed, allowing both graphical (Martin, et al., 1983) and numerical (Parrott, 1970) estimation of the standard deviation of the geometric mean size. The slightly widened distribution of the Starch 1500 is reflected by larger values for the standard deviation. Starch 1500 Lot $C$ had a calcualted mean $\sigma_{g e o}$ of 2.13, compared with 1.91 for Starch 1551 ( $p<0.0001)$. Duncan groupings for the four starches are shown in Table 4.

Figure 2 shows the particle size distributions for lot C of Starch 1500 and Starch 1551. The distributions appear similar; Starch 1500 , however, is weighted slightly toward both the smallest and largest sizes. The smallest sizes (through US Sieve \#400) likely represent many intact corn starch grains, which are usually in the size range below 35 microns (Boylan, et al., 1986).

Table 4

Duncan's Multiple Range Test of Geometric Mean Particle Size Standard Deviation (Particle Size Uniformity $\sigma_{\text {geo }}$ ) for Starches 1500 and 1551

\begin{tabular}{|l|c|c|c|c|}
\hline Starch Type & $1500(\mathrm{~B})$ & $1500(\mathrm{~A})$ & $1500(\mathrm{C})$ & 1551 \\
\hline Mean $\sigma_{\text {geo }}$ & 2.26 & 2.17 & 2.13 & 1.91 \\
\hline Duncan Grouping & $\mathrm{A}$ & $\mathrm{B}$ & $\mathrm{B}$ & $\mathrm{C}$ \\
\hline
\end{tabular}

(means with the same letter are not significantly different) 
Figure 2

Particle Size Analysis of Starches 1500 and 1551

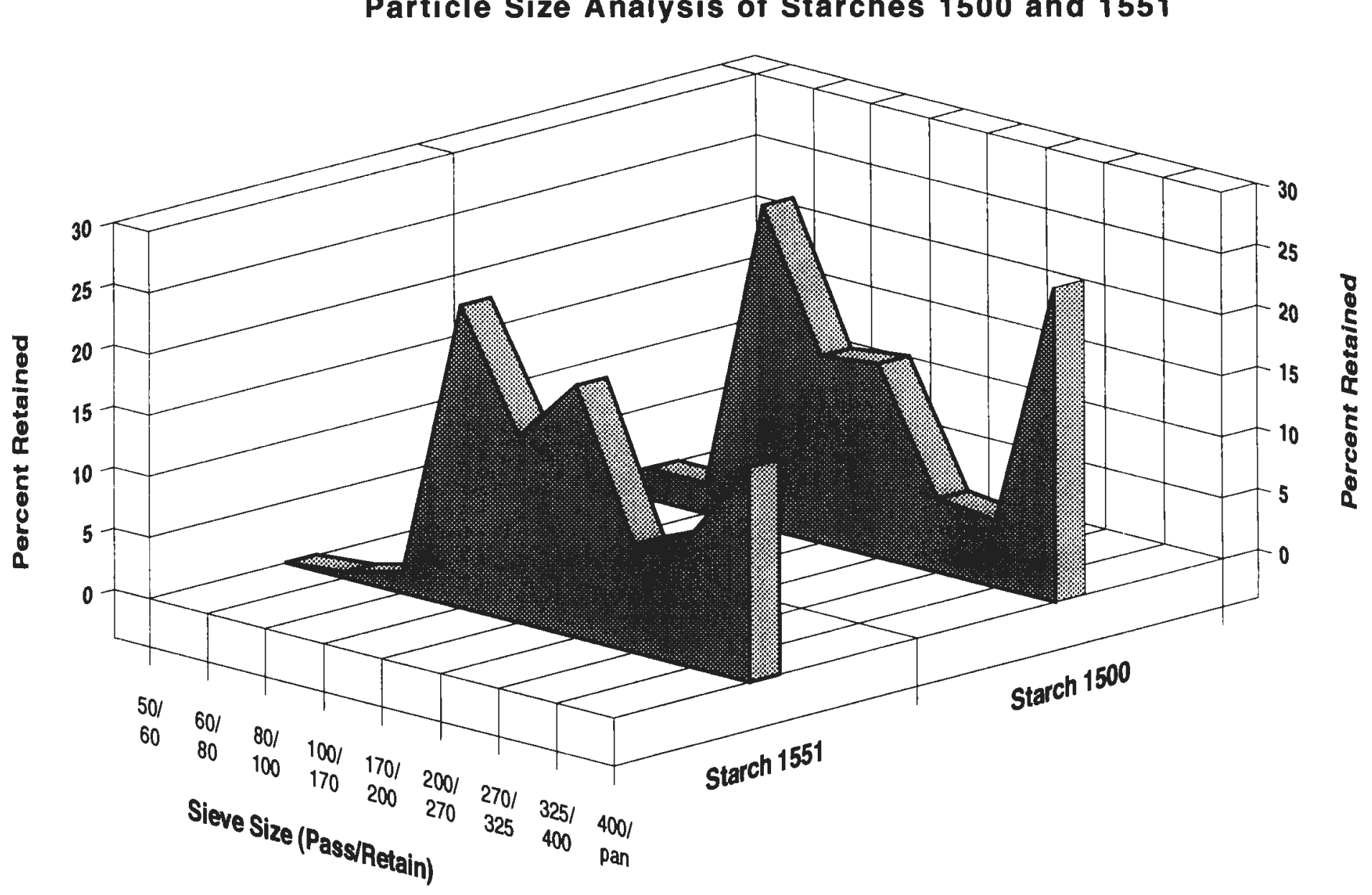




\section{Analysis of Granulations}

Particle Size

Three different particle size parameters are evaluated for each batch. The volumesurface mean diameter $d_{v s}$ is particularly useful because it is inversely related to the specific surface of the material. A decrease in $d_{v s}$, therefore, indicates a commensurate increase in surface area. Parrott (1970) recommends its use when the surface properties of a material may be important.

Table 5 shows sieve data for individual treatments and the resulting mean size parameters $d_{m}, d_{g e o}$ and $d_{v s}$. Each datum represents the mean of three determinations. The median size $d_{m}$ is determined graphically; the other are calculated. Of the three, the volume-surface mean $d_{v s}$ shows the best linear relation to the three major factors STARCH, MIXIER and SPEED $\left(r^{2}=0.996, p<0.0001\right)$. All three major factors were statistically significant as well as the two-factor interaction between MIXER and SPEED.

Starch 1500 made granulations with larger particle sizes than Starch 1551. Mean $d_{v s}$ values were $570 \mu$ and $537 \mu$, respectively. The difference was significant $(p=0.04)$.

Fielder granulations were significantly more coarse $(p=0.0005)$, with average $d_{v s}$ values of $627 \mu$ and $480 \mu$ for the Fielder and PowerMix, respectively.

High chopper speeds during processing resulted in significant reductions in particle size. On average, particle sizes were approximately one-fourth smaller (641 $\mu$ vs. $465 \mu)$ when high speeds were used. This difference was significant $(p=0.0003)$. Reductions in particle size caused by high chopper speeds are more pronounced with the PowerMix than with the Fielder. This interaction effect can be seen in Figure 3. The two lines are not parallel; the PowerMix shows a greater decrease $(p=0.007)$. 
The evidence here suggests that the PowerMix is a higher intensity mixer than the Fielder. The qualitative effect of increasing the chopper speed in both mixers is a reduction in particle size. The PowerMix produces smaller sizes than the Fielder at both tested speeds; in fact, the PowerMix at low speed is as effective as the Fiedler at high speed.

Granulation particle size is a gauge of the extent of agglomeration by virtue of the kinetics of granulation growth (Usteri, 1989). Starch 1500 made granulates that were slightly larger in size than those made with Starch 1551. The difference (about 6\%) was small, but nevertheless indicates that as a binder in a wet granulation process, there is no reason to believe Starch 1500 to be inferior to Starch 1551 . 
Figure 3

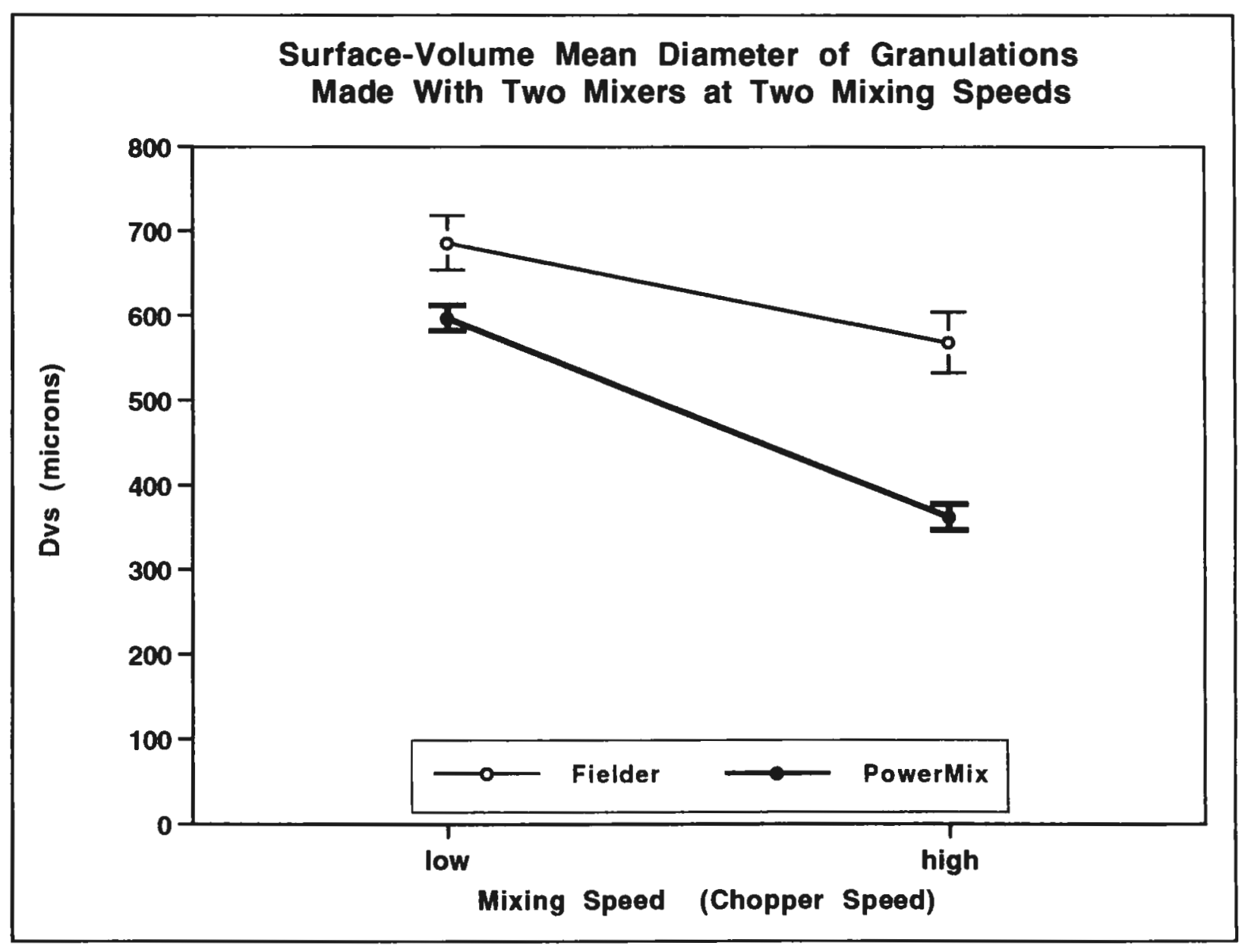




\section{Particle Size Unifomity}

The standard deviations of the geometric mean particle sizes $\sigma_{g e o}$ are use to indicate granulation uniformity. An analysis of the data shows that PowerMix granulations have better size uniformity than those made with the Fielder $(p=0.004)$. This is true at both low and high speeds. High mixing speeds themselves also increased uniformity $(p=0.004)$. Starch type, however, had no effect on uniformity.

The average geometric standard deviations for each group are shown below. Lower values correspond to a more uniform particle size distribution.

\section{Table 6}

Mean Standard Deviations of the Geometric Mean Particle Size $\sigma_{g e o}$ (Particle Size Uniformity) for Two Mixers at Two Speeds

\begin{tabular}{|c|c|c|c|}
\hline $\begin{array}{c}\text { PowerMix } \\
\text { High Speed }\end{array}$ & $\begin{array}{c}\text { PowerMix } \\
\text { Low Speed }\end{array}$ & $\begin{array}{c}\text { Fielder } \\
\text { High Speed }\end{array}$ & $\begin{array}{c}\text { Fielder } \\
\text { Low Speed }\end{array}$ \\
\hline 1.68 & 2.19 & 2.32 & 2.49 \\
\hline
\end{tabular}

Sieve data is plotted in Figure 4. As mixing speeds are increased, it can be seen that smaller particles become more involved in the agglomeration process. The smallest size range decreases from a high of $10.17 \%$ (Fielder at low speed) to $0.78 \%$ (PowerMix at high speed). At the same time, there is a reduction in the proportion of large particles from $11.28 \%$ (Fielder at low speed) to $0.47 \%$ (PowerMix at high speed). The function of the 
chopper, then, is to involve the smallest particles in the agglomeration process while preventing agglomerates from becoming too large. In this way, particle is controlled by the chopper, resulting in better uniformity.

\section{Bulk Density}

Bulk density measurements include free-flowing material and packed (tapped) densities. The free-flowing (loose) densities of the granulations were essentially identical for all batches. None of the major factors, nor any two-factor interactions, were statistically significant. The results differed, however, for the tapped densities. Both MIXER and SPEED had significant effects ( $p=0.01$ and 0.005 , respectively). There was no difference between starches, nor were there any interaction effects.

Figure 5 shows that PowerMix granulations had higher tapped densities than those made with the Fielder $(p=0.01$ ). This may correspond to the smaller mean particle sizes of PowerMix materials, resulting in smaller intergranular spaces and therefore higher packing densities.

Granulations made at lower speeds have higher packing densities $(p=0.005)$, even though lower speeds resulted in larger particle sizes. This appears to contradict the results seen above for mixer type, but this apparent anomaly may be resolved by the fact that low mixing speeds resulted not only in more large sizes, but also in a higher percentage of fines (see Figure 4 and Table 5), which fill intergranular voids between large particles, allowing more efficient packing. 
Figure 4

\section{Particle Size Distributions of Granulations Made With}

Two Mixers at Two Mixing Speeds

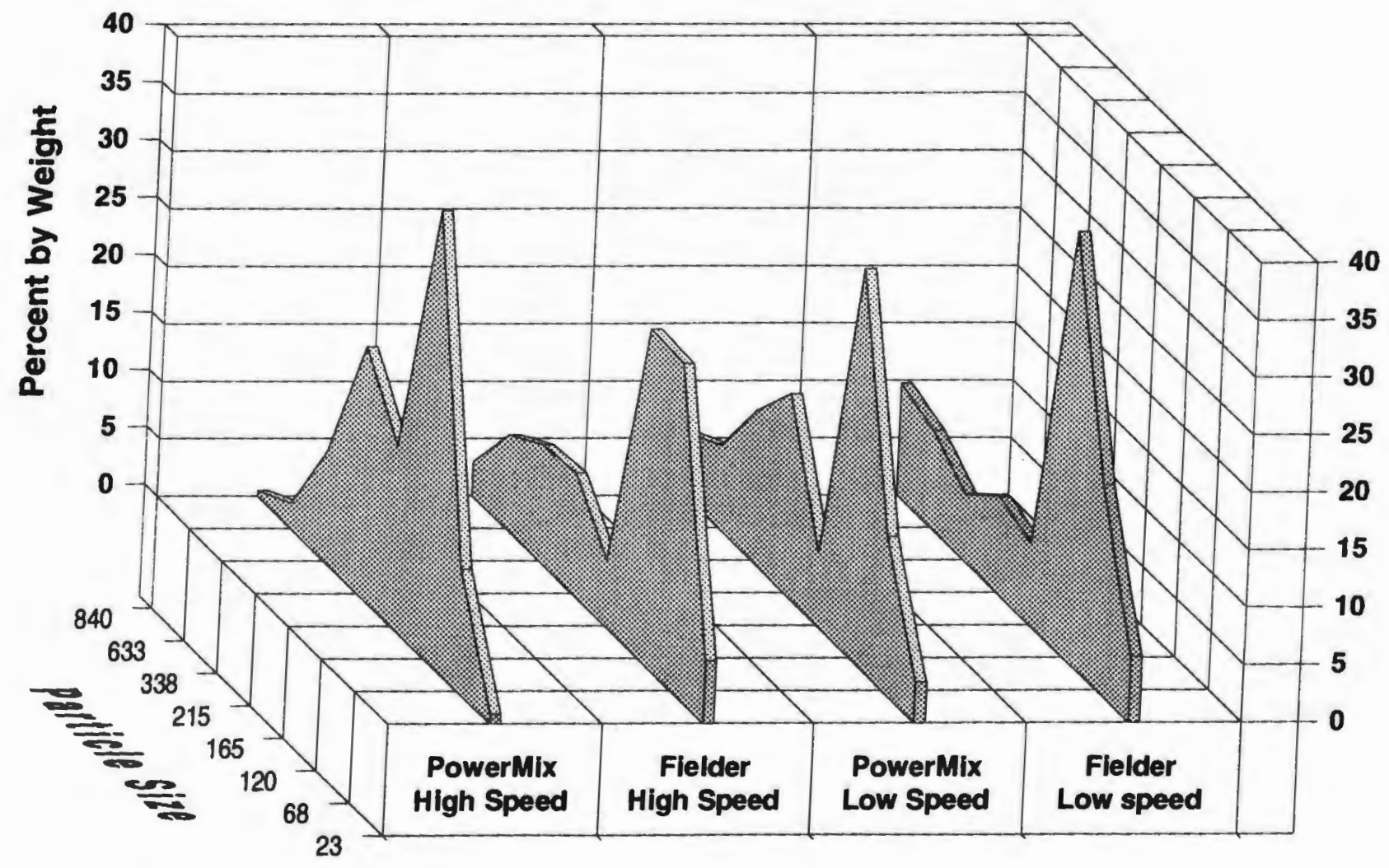




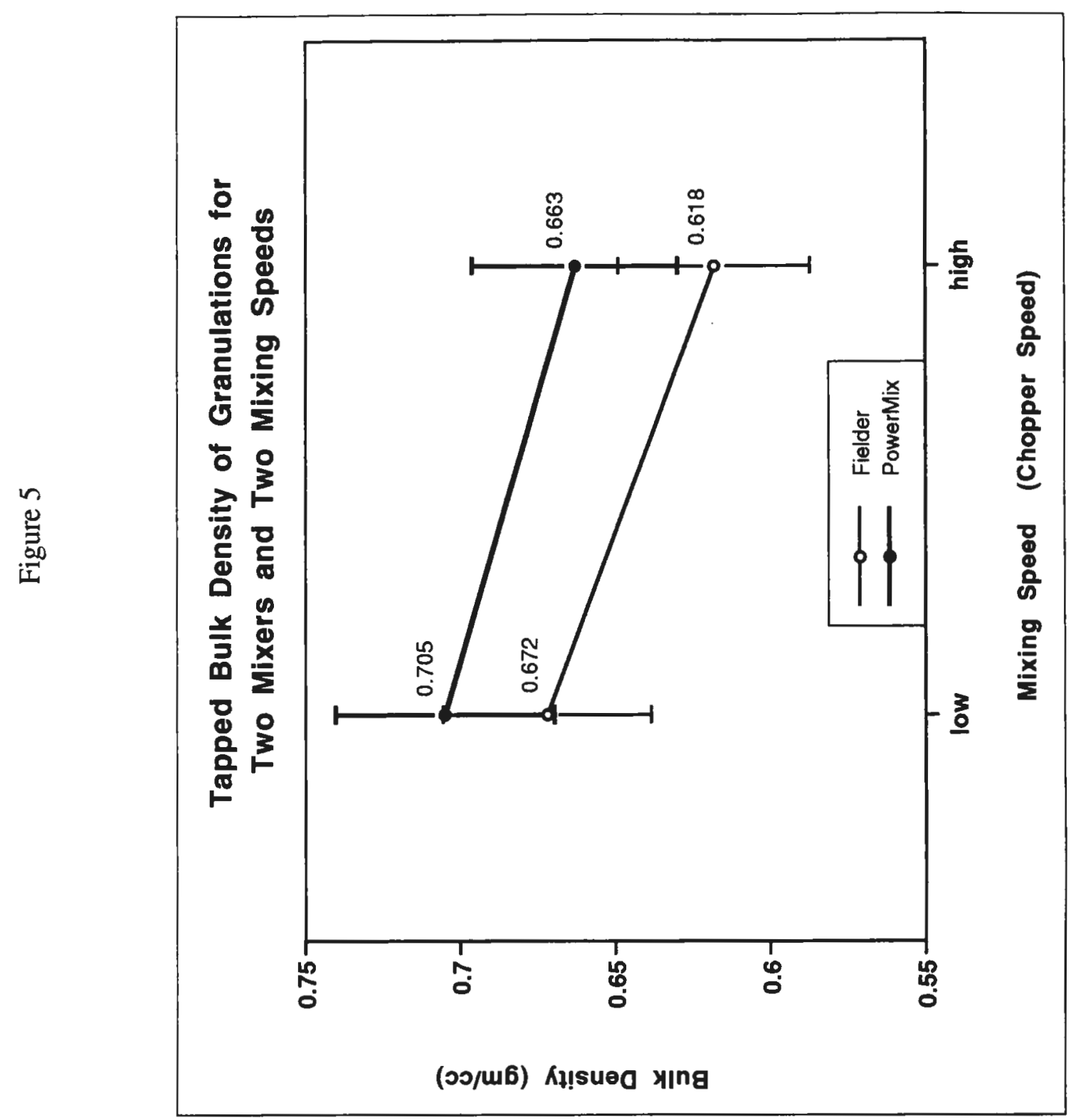




\section{Flow Properties}

The flowability of granulations was evaluated by two methods, the compressibility index and static angle of repose measurements. These two parameters show fairly good agreement, as shown in Figure 6.

The compressibility index (CI) indicates the ease with which a material can be induced to flow. Values below $15 \%$ usually give rise to good flow characteristics, but readings above $25 \%$ indicate poor flowability (Lachman and Lieberman 1986, p.67). Scheffe's Tests revealed no statistically significant differences between the two mixers, the two starches or the two speeds. A regression fit to the three main factors showed a marginal significance only between starches $(p=0.04)$. Interestingly, however, only four granulations had CI values below the benchmark $25 \%$. Of these, three of the four were made in PowerMix and three of the four were made using high speeds.

Static angle of repose measurements were carried out in replicates of three to five trials. Significant differences were found for all major factors and two-factor interactions. The results confirm the suggestions based on the compressibility indices that better granulation flow could be achieved using Starch $1500(p<0.0001)$, the PowerMix $(p<0.0001)$ and higher mixing speeds $(p<0.0001)$. Of the three factors, choice of starch had the greatest effect (about a 7\% difference), followed by mixer type and mixing speed. The results for both mixers as a function of mixing speed can be seen in Figure 7. The non-parallel nature of the two lines indicates the interaction between mixer and speed $(p<0.0001)$.

Values for angle of repose not more than $30^{\circ}$ usually indicate a freely-flowing material and angles $40^{\circ}$ or more suggest a poorly flowing material (Lachman and Lieberman 1986, p.67). All PowerMix batches had angles less than forty degrees. Fielder batches had the three worst results, including two batches exceeding forty degrees. The results are shown in Table 7. 
Table 7

Angle of Repose Results for Granulations (in degrees)

\begin{tabular}{|l|c|c|c|c|c|c|c|c|}
\hline MIXER & $\begin{array}{c}\text { Power } \\
\text { Mix }\end{array}$ & $\begin{array}{c}\text { Power } \\
\text { Mix }\end{array}$ & $\begin{array}{c}\text { Power } \\
\text { Mix }\end{array}$ & $\begin{array}{c}\text { Power } \\
\text { Mix }\end{array}$ & Fielder & Fielder & Fielder & Fielder \\
\hline STARCH & 1500 & 1500 & 1551 & 1551 & 1500 & 1500 & 1551 & 1551 \\
\hline SPEED & low & high & high & low & high & low & high & low \\
\hline Trial 1 & 37.05 & 37.23 & 38.66 & 37.82 & 35.26 & 38.96 & 40.05 & 43.85 \\
\hline Trial 2 & 36.32 & 34.52 & 39.27 & 39.27 & 36.63 & 39.31 & 37.82 & 45.00 \\
\hline Trial 3 & 35.04 & 36.57 & 37.36 & 38.56 & 36.21 & 40.74 & 39.05 & 45.00 \\
\hline MEAN & 36.14 & 36.11 & 37.77 & 38.55 & 36.03 & 39.67 & 38.97 & 44.62 \\
\hline
\end{tabular}


Figure (6)

The Graph of Angle of Repose vs. The Compressibility Index

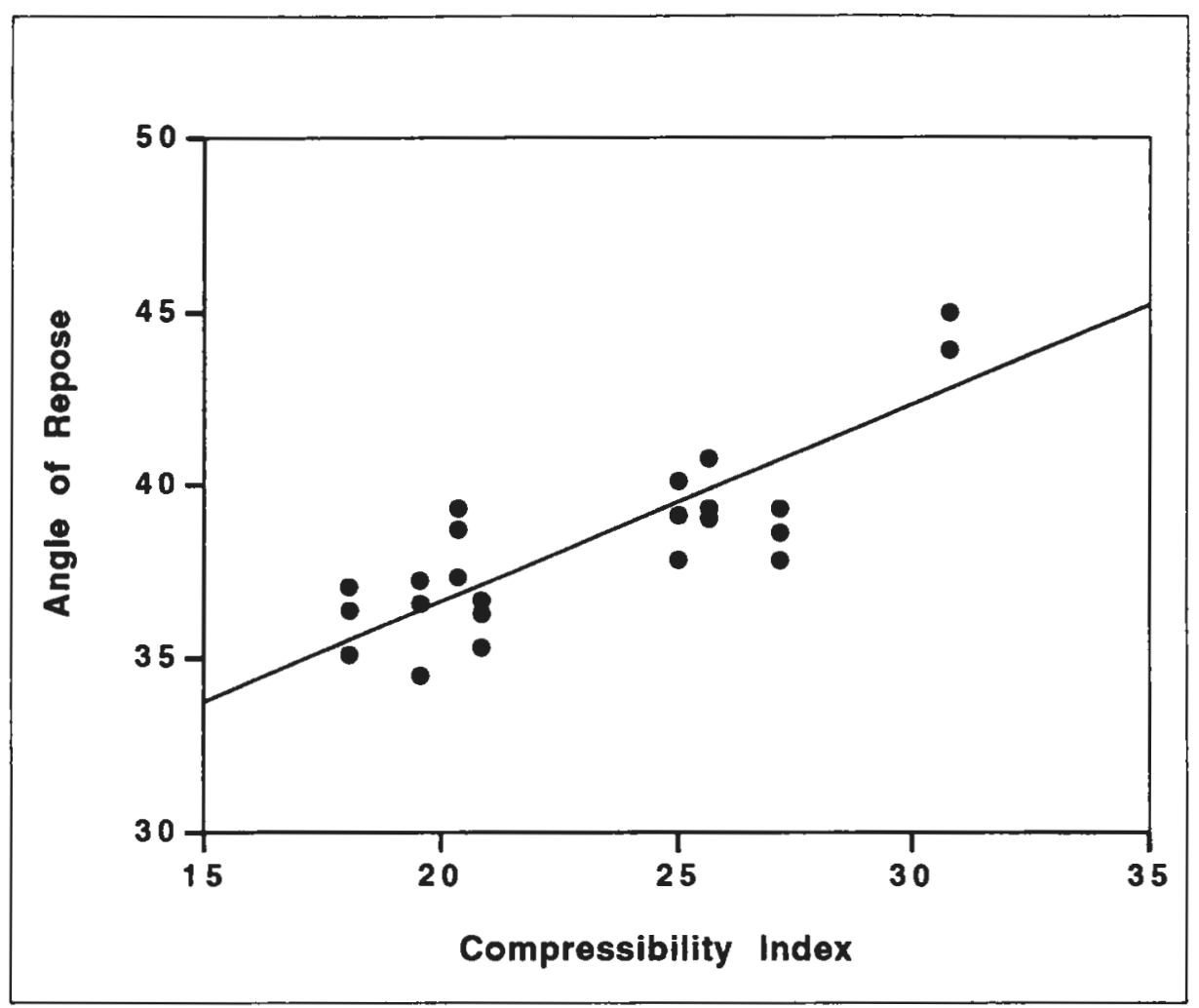


Figure 7

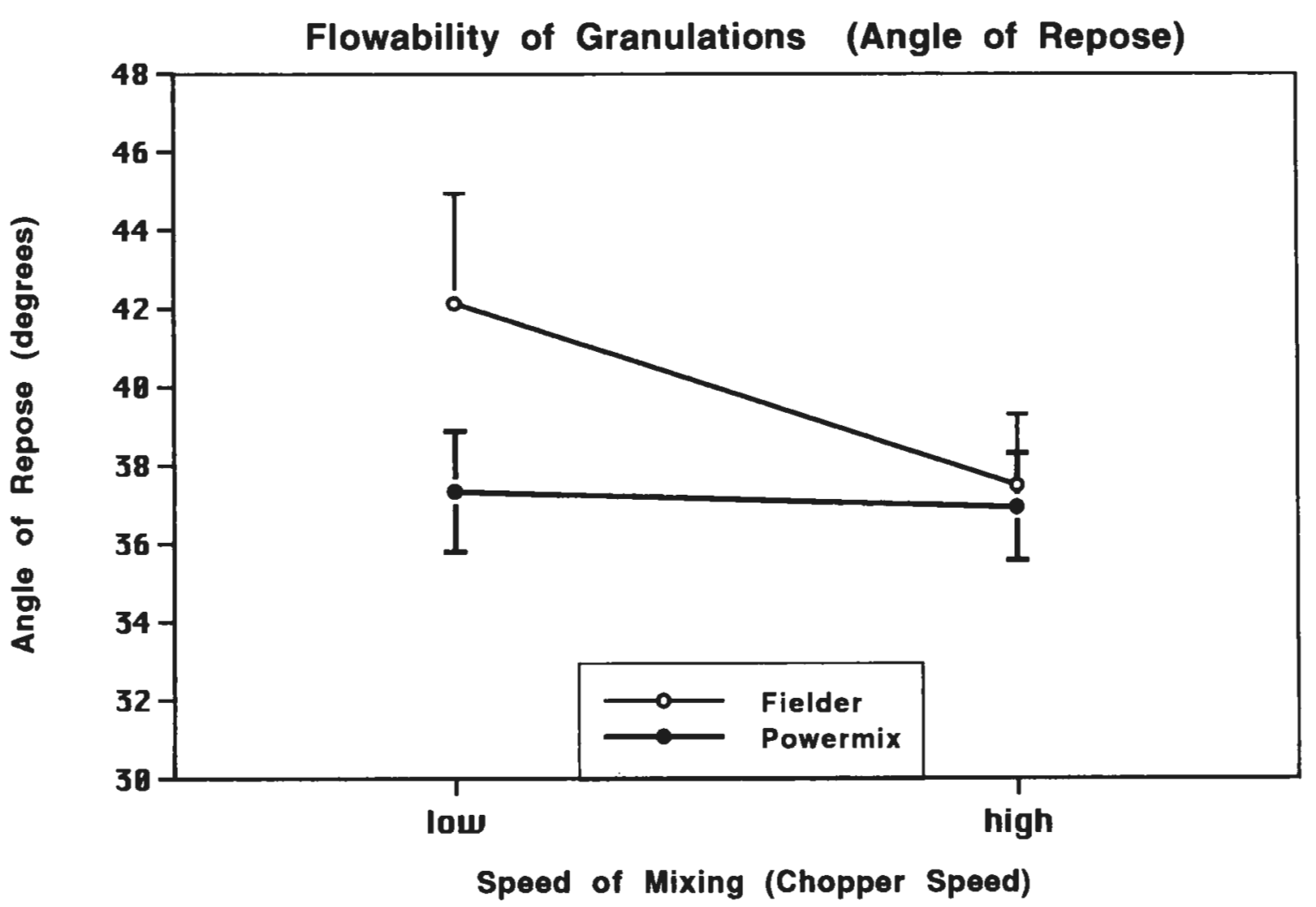

The graph shows the following effects:

- The Ross Powermix made granulations with better flowability

- The effect of chopper speed is significant (higher speed is better)

- The effect of mixing speed depends upon which mixer is used 


\section{Evaluation of Tablets}

Compression

All tablets were compressed at forces of $12 \mathrm{kN}( \pm 1$, range). The ability of the granulations to form hard, durable tablets was then gauged by the cohesion index.

Tablets made with both Starch 1500 and Starch 1551 were generally soft, with maximum hardnesses in the $3 \mathrm{~kg}$ range. Nonetheless, significant differences between treatments could be discerned. Cohesion indices ranged from a minimum of 0.159 to a maximum of $0.316 \mathrm{~kg} / \mathrm{kN}$.

Mixing speed had the greatest effect on compactability $(p<0.0001)$. Tablets made using higher mixing speeds were $74 \%$ harder, on average.

Formulations using Starch 1500 were 36\% harder than those made with Starch 1551 , a difference which was statistically significant $(p<0.0001)$.

The type of mixer used also had a significant $(p=0.016)$, although smaller, effect. PowerMix granulations formed tablets that were only $7 \%$ harder, on average.

The MIXER $\cdot S P E E D$ interaction was only marginally significant $(p=0.055)$. The PowerMix made harder tablets if both mixers used low speed, but at high speeds there was no difference. The effects of speed and the influence of the mixer type can be seen in a graph of the two-factor interaction Figure 8. 


\section{Figure 8}

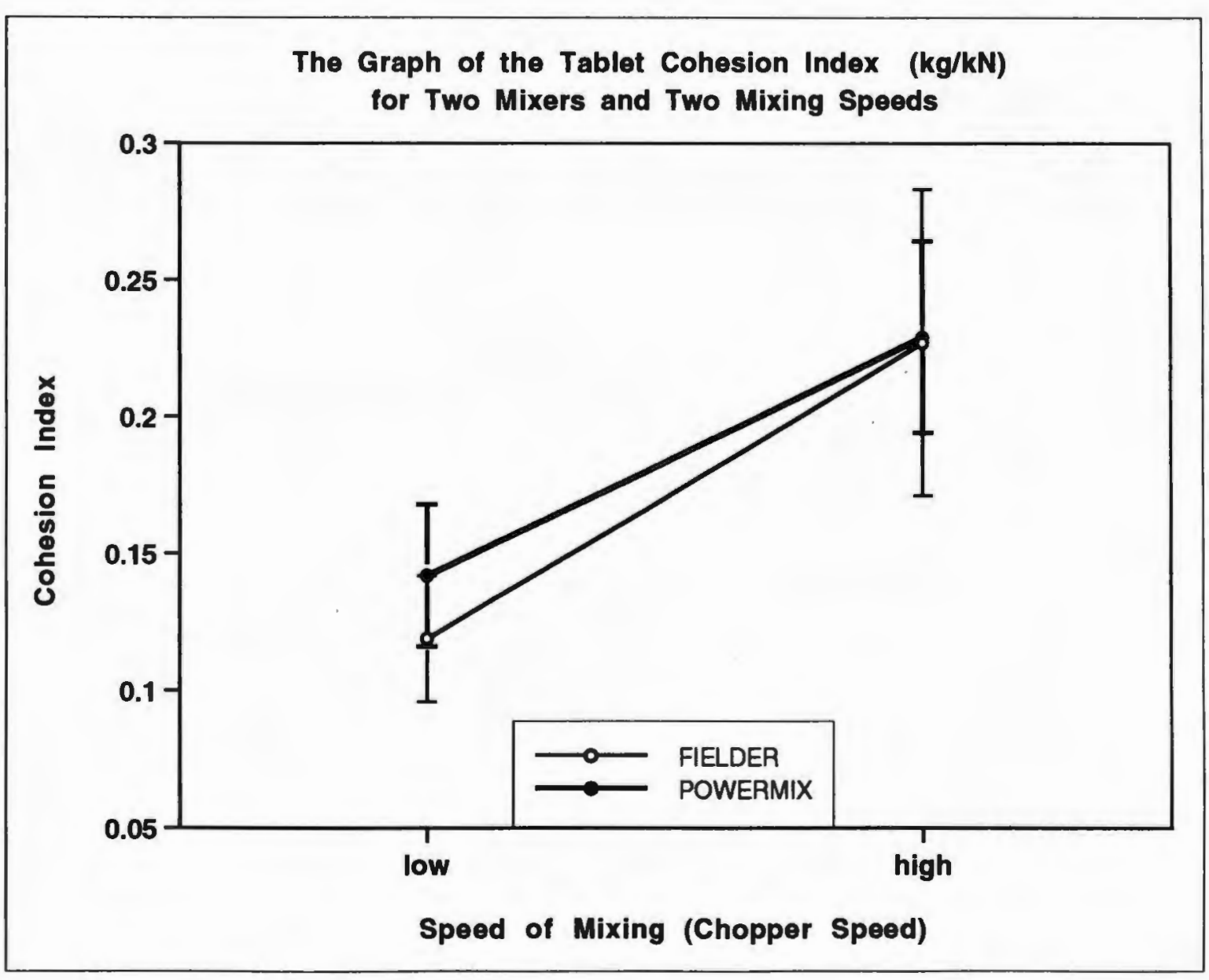




\section{Tablet Ejection}

Ejectional force data showed a good regression fit to a linear model using the three main factors and their two-factor interactions $\left(r^{2}=0.975\right)$. All six terms in the model were significant. The most significant among these was the mixer used $(p<0.0001)$, with ejection forces during tableting being higher for PowerMix granulations.

Mixing speed was the next most important factor $(p<0.0001)$, with higher mixing speeds resulting in higher ejectional forces.

The type of starch also had a significant effect $(p<0.0001)$, with Starch 1500 requiring $17 \%$ more force, on average, to remove the tablet from its die.

Among two-factor interactions, SPEED•MIXER was the most significant $(p<0.0001)$. Changing from low speed to high speed using the PowerMix resulted in a greater increase in ejection force than a similar change using the Fielder, suggesting a greater intensity of mechanical action.

The difference in ejection forces measured between mixers at both low and high speeds can be explained by a simultaneous examination of the relative surface areas of the granulations. The volume-surface mean particle diameter $d_{v s}$ is inversely proportional to the specific surface of a granulation. As $d_{v s}$ decreases, the surface area per unit volume of material increases. When $d_{v s}$ data from Table 5 is plotted with the left $\left(d_{v s}\right)$ ordinate in decreasing order in Figure 9, we see the relative increase in surface area that results. The increase in surface area closely parallels the increase in the amount of ejection force required, particularly in the case of the Fielder. This suggests that as the added lubricant is distributed over a greater surface area, its effect is diluted. 
Figure 9

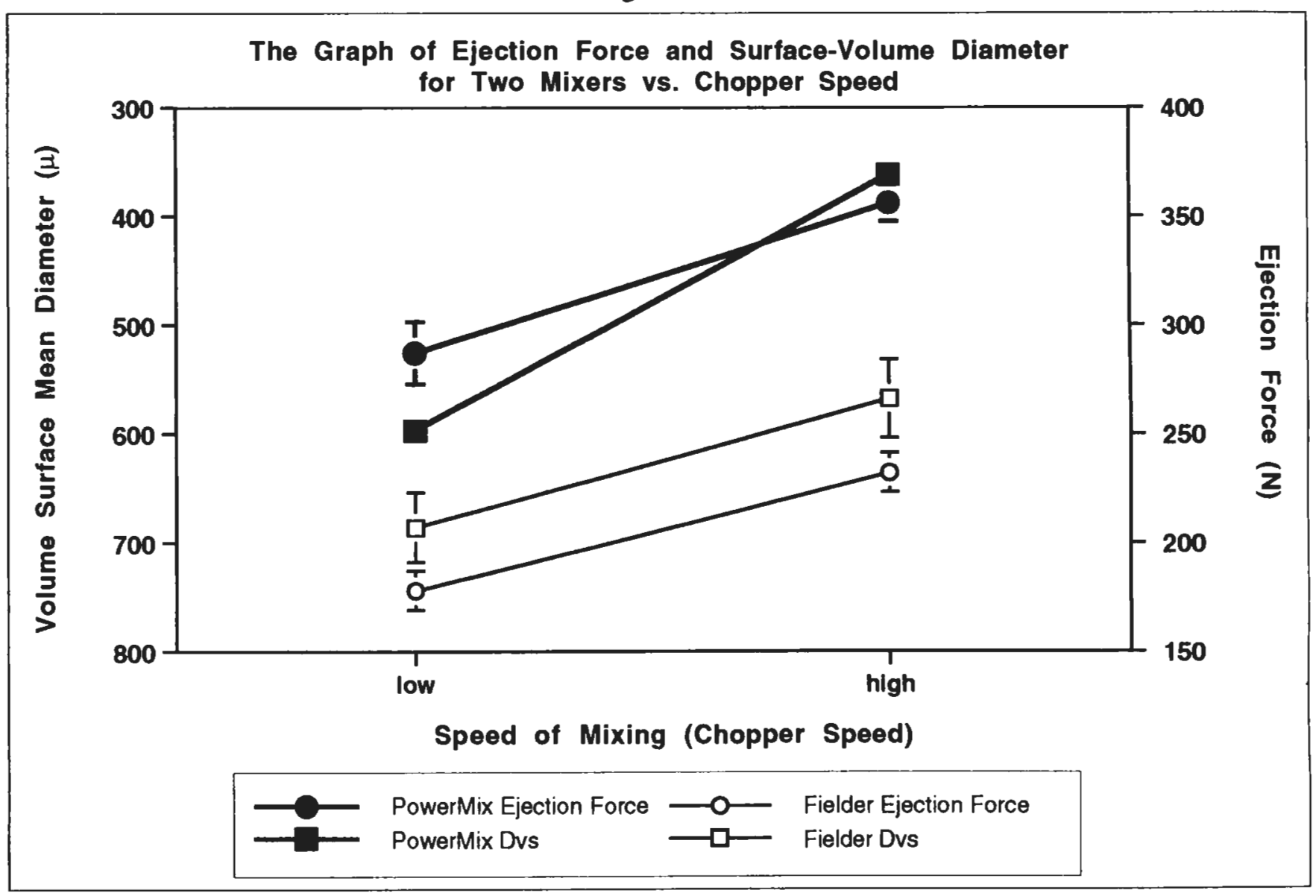

- The left (Dvs) ordinate is plotted in reverse (decreasing) order

- The difference between mixers is given by line separation

- $\quad$ The effect of mixing speed is given by the positive slopes

- MIXER and SPEED are significant for both ejection force $(p<0.0001)$ and Dvs $(p=0.007)$ 
Tablet Weight Variation and Compression Force Variability

Tablet weight variation and the variability in compression force are useful parameters for evaluating tablet quality. Both tablet weight and peak compressional force variabilities are measured in terms of the relative standard deviation (RSD). Compression forces were measured for each batch at three or four different pressure settings for 25 tablets each. Ten tablets were weighed and their relative standard deviations calculated. Figure 10 shows that the two parameters are linearly correlated.

Compression force results Figure 11 show that the speed of mixing had a significant effect $(p<0.0001)$. High mixing speeds were effective in reducing the variability during tableting, causing a mean reduction of $51 \%$ (from $5.8 \%$ to $2.8 \%, \mathrm{n}=13$ ).

The type of starch had a less pronounced effect $(p=0.025)$. Starch 1500 reduced variability by $24 \%$ (3.7\% vs. $4.9 \%)$ when compared with Starch 1551 .

The PowerMix made more consistent tablets than the Fielder $(p=0.017)$, causing a $21 \%$ reduction in compressional variability (3.7\% vs. $4.7 \%)$.

There were no significant interaction effects. 
Figure 10

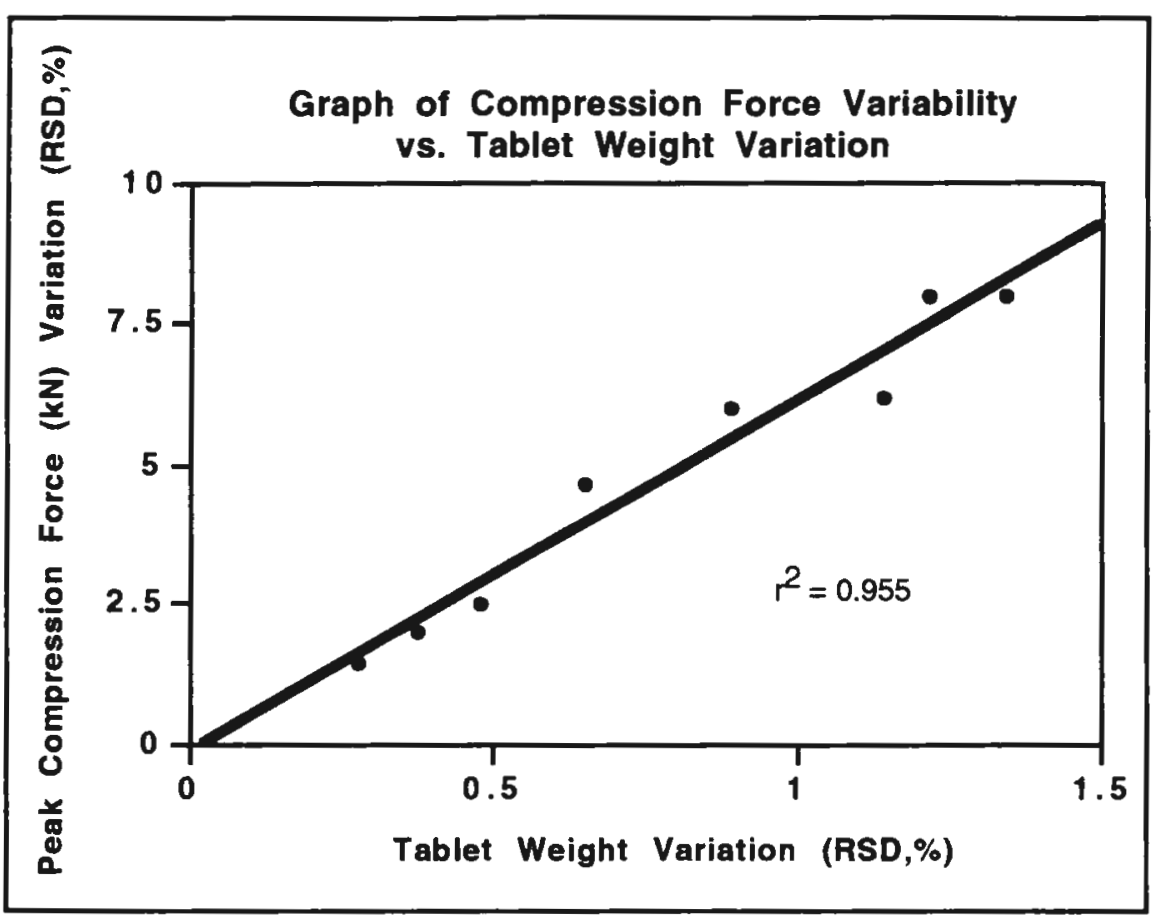


Figure 11

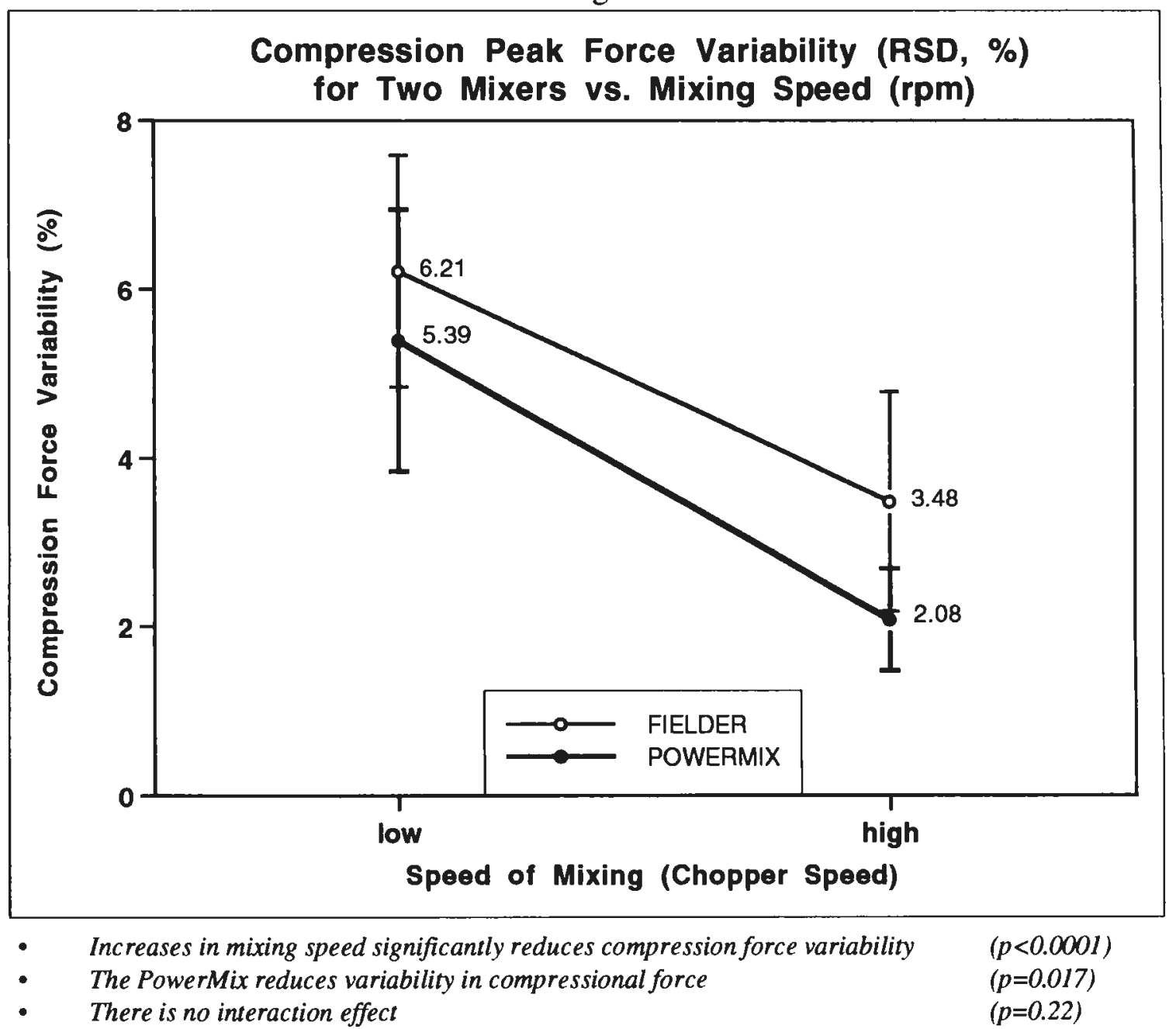


Tablet Friability

Tablet friabilities for all batches tested were too high for the tablets to be considered acceptable dosage forms, although the values differed widely. Friabilities ranged from a high of $13.9 \%$ to a low of $1.50 \%$, where values exceeding $1 \%$ are generally regarded as unacceptable for a good tablet (Lachman and Lieberman, 1986). These high friabilities reflect the high $(90 \%)$ percentage of acetaminophen, which has poor binding properties and displays a high degree of elastic recovery following compression (Swarbrick, et al. 1988).

Despite the overall poor results, results show that chopper speed was instrumental in producing tablets of improved strength $(p=0.006)$. Mean friabilities were $11.1 \%$ at low speed versus $3.3 \%$ at high speed. Figure 12 shows the results for the two mixers. The PowerMix made slightly better tablets than the Fielder (5.7\% vs.8.7\%), but the difference was not significant $(p=0.07)$. There was also very little difference between starches.Starch 1500 performed slightly better than Starch 1551 (5.5\% vs. $8.9 \%, p=0.055)$.

\section{Disintegration Time}

Disintegration times for all tablets were quite short. The longest recorded time for any one tablet was 62 seconds; the shortest was approximately 10 seconds. These results are not surprising, considering their soft, friable consistency. Disintegration time was not related to tablet density, but was found to be longer with decreases in tablet friability (Figure 13) and increases in tablet hardness.

All three main factors had significant effects upon the disintegration time of tablets. Longer disintegration times resulted from using Starch 1500, choosing the PowerMix over 
Figure 12

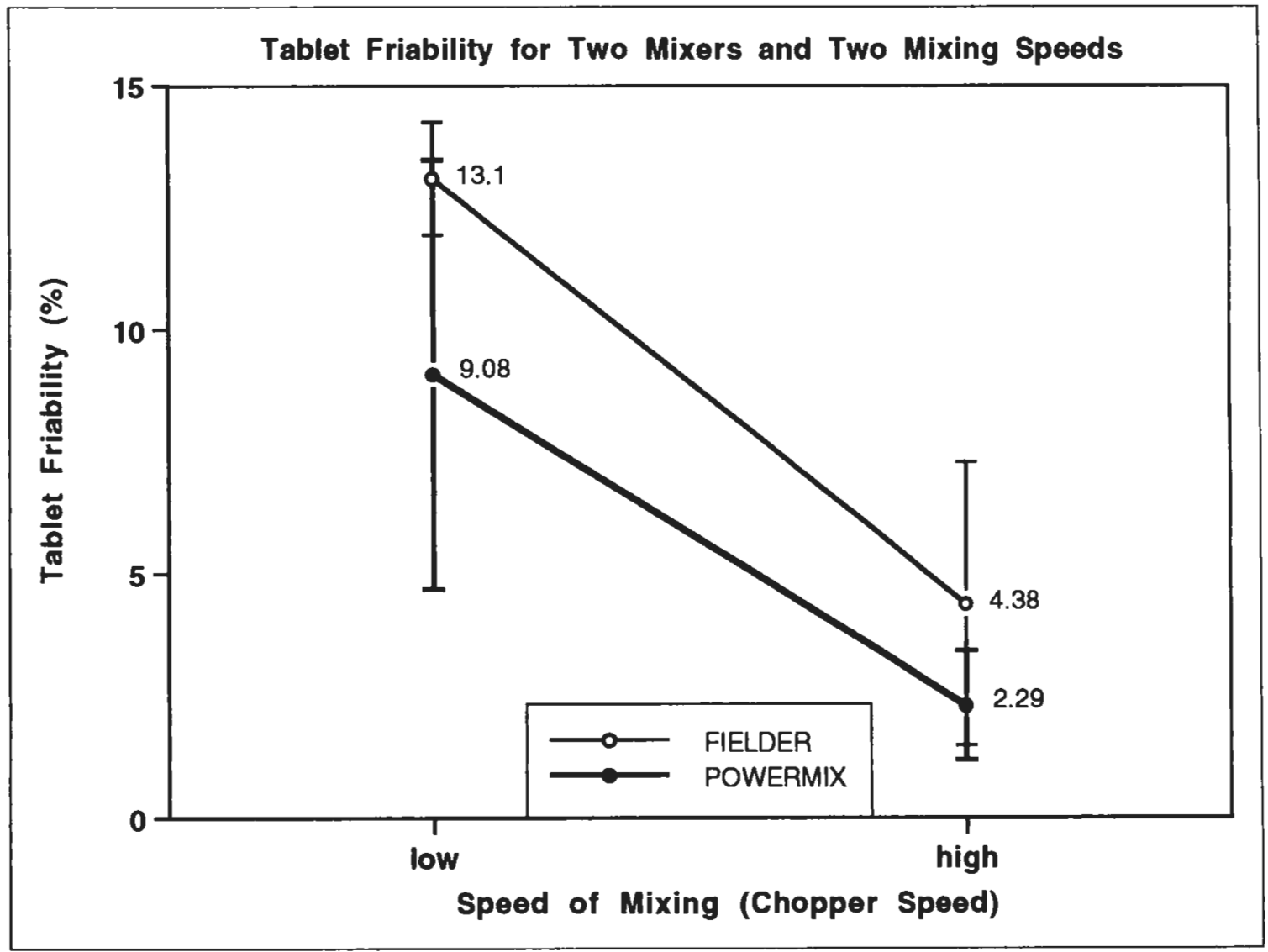

The graph shows the following effects:

- PowerMix granulations made less friable tablets

$(p=0.055)$

- High chopper speeds significantly reduced friability

$(p=0.006)$

- The effect of mixing speed does not depend upon which mixer is used $\quad(p=0.45)$ 
the Fielder, and using high mixing speeds. The effect of speed for the two mixers is seen in Figure 14. All main and two-factor interactions were significant at $p<0.0001$.

Figure 13

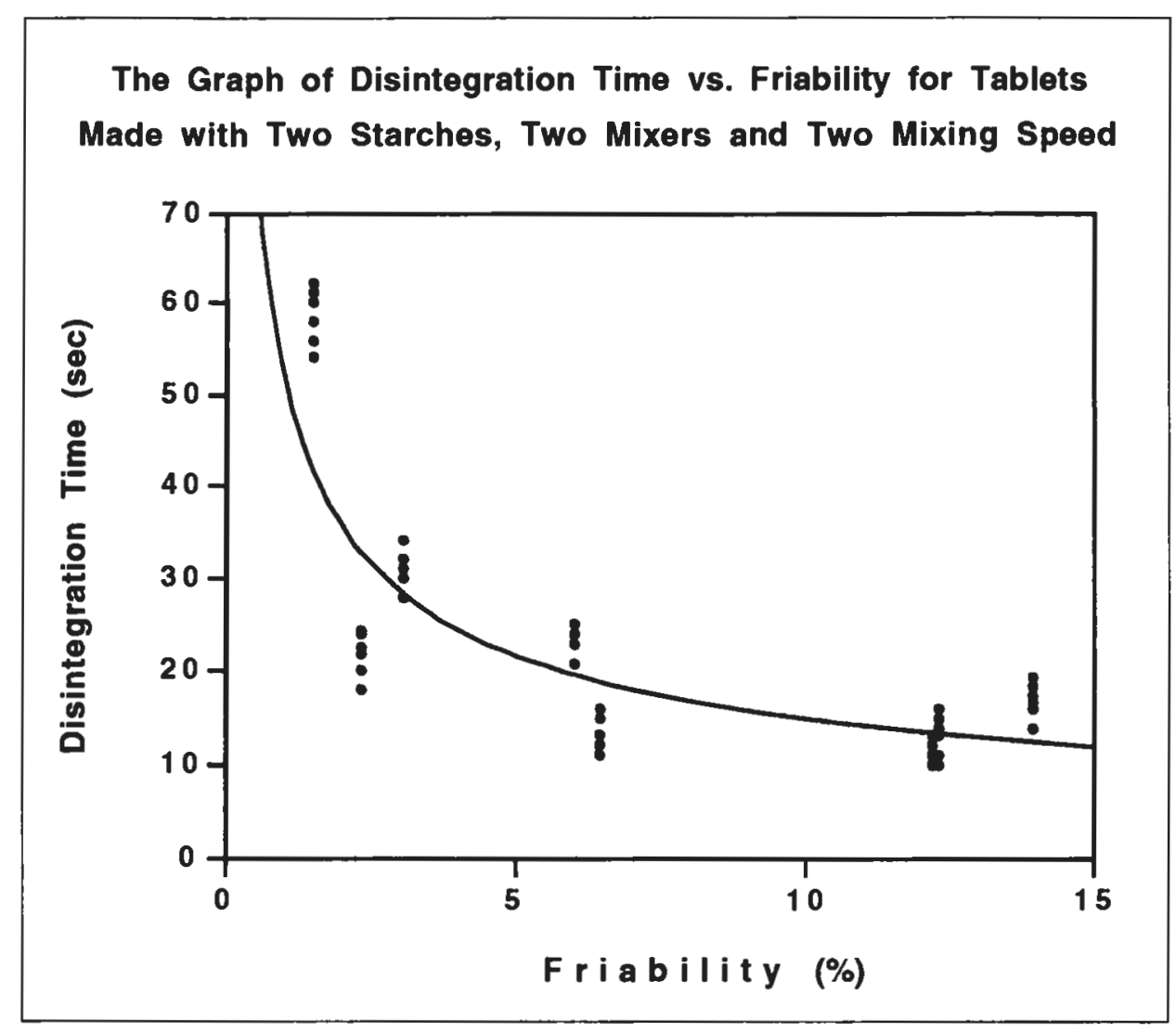


Figure 14

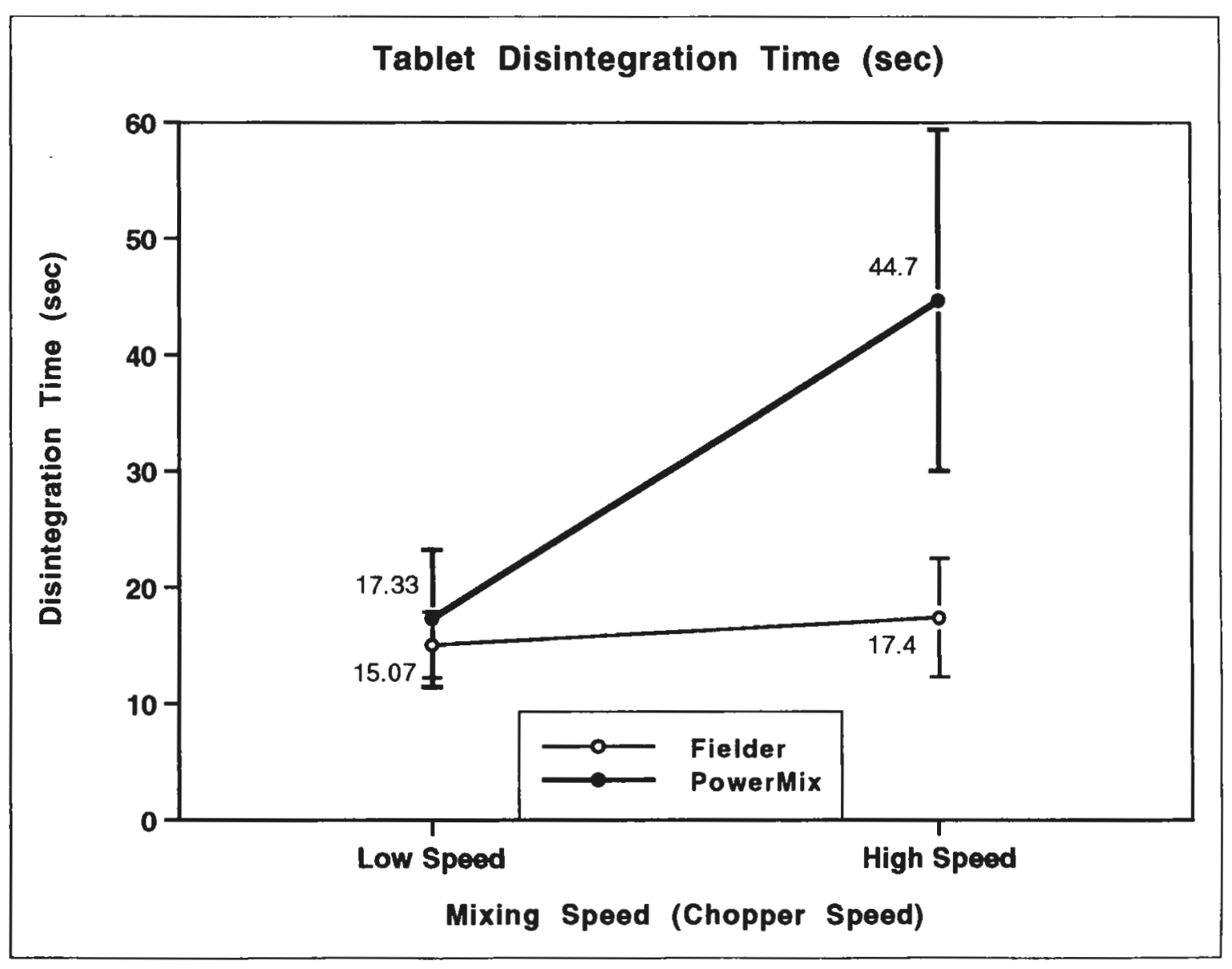




\section{Conclusions}

Ten response variables were evaluated. These characterized finished granulates, tableting process parameters and finished tablets. Mixing speed had a significant, often dramatic effect upon all of them. In each case except one (ejection force), the effect of increasing mixing speed during the granulation process was to improve the response.

Almost as important as mixing speed as a factor was the difference between the two mixers. Once again, with the exception of disintegration time, every response variable measured favored the PowerMix over the Fielder. The statistical significance of each was not necessarily as high, and in one case it was marginal (Friablility, $p=0.07$ ), but the inference is clear.

There were important differences between the two starches. Seven of the ten response variables revealed significant differences; three did not. Of the seven, three parameters were only marginally significant. The clearest differences between the two excipients were seen when flowability of the granulations and tablet strength were considered. 


\section{REFERENCES}

Boylan J.C., Cooper J., Chowhan Z.T., Lund W., Wade A., Weir R.F., Yates B.J., Handbook of Pharmaceutical Excipients, American Pharmaceutical Association, Washington, D.C., 1986.

Carr R.L. (1965) Evaluating Flow Properties of Solids, Chemical Engineering, 72, 163168.

Hines W.W., Montgomery D.C. (1990) Probability and Statistics in Engineering and Management Science, Third Edition, John Wiley \& Sons, New York.

Lachman L., Lieberman H.A., Kanig J.L. (1986) The Theory and Practice of Industrial Pharmacy, Third Edition, Lea \& Febiger, Philadelphia.

Liu C.H., Chen S.C., Lee Y.C., Sokoloski T.D., Sheu M.T. (1994) Directly Compressible Acetaminophen Compositions Prepared by Fluidized-Bed Granulation, Drug Development and Industrial Pharmacy, 20(11), 1911-1922.

Martin A., Swarbrick J., Cammarata A., Chun A.H.C. (1983) Physical Pharmacy, Third Edition, Lea \& Febiger, Philadelphia.

Patel N.K., Poola N.R., Babar A., Plakogiannis F.M. (1989) Fluidized-Bed Agglomeration of Acetaminophen; Direct Compression of Tablets and Physiologic Availability, Drug Development and Industrial Pharmacy, 15(8), 1175-1198. 
Parrott E.L. (1970) Pharmaceutical Technology, Burgess Publishing Company, Minneapolis.

Salpekar A.M., Denton L.E. (1987) Direct Tableting Acetaminophen Compositions, United States Patent \#4,661,521.

Salpekar A.M., Denton L.E. (1987) Direct Tableting Acetaminophen Compositions, United States Patent \#4,757,090.

Swarbrick J., Boylan J.C., ed (1988) Encyclopedia of Pharmaceutical Technology, Marcel Dekker, New York.

Usteri M., Leuenberger H. (1989) Agglomeration of Binary Mixtures in a High-Speed Mixer, International Journal of Pharmaceutics, 55, 135-141.

Vogel S.T. (1984) Directly Compressible Acetaminophen Granulation, United States Patent \# 4,439,453. 
Manuscript III

The Influence of Process Variables on the High-Speed Wet Granulation of Two Acetaminophen-Pregelatinized Starch Products

Using the Ross PowerMix ${ }^{\circledR}$ 
ABSTRACT

A full factorial design experiment with four factors at two levels is carried out to wet granulate a $90 \%$ acetaminophen-10\% pregelatinized starch combination. The experiment uses a high-shear mixer design with two different quantities of water addition, two types of pregelatinized starch excipient. Following wet granulation, the finished granules are dried by two different methods. The purpose of the experiment is to determine if a purported defect in Starch 1500 exists when subjected to a high-shear process and to what extent speed of mixing may be an important variable.

Nine responses were measured, including granulation and tablet quality, in conjunction with tableting parameters from an instrumented tablet press.

The results show that the high-speed capability of the mixer is the critical factor, affecting and improving nearly every response measured. The uniformity of size, flowability, compactability, tablet durability and disintegration time were all strongly affected by mixing speed increases. Almost as important is the amount of water used in the process. Choice of starch favored Starch 1500 , contrary to any notion of inferiority. The method of drying was essentially of no consequence, with most of the properties of the granules and tablets governed by preceding steps. 


\section{INTRODUCTION}

Manuscript II used two different mixer designs, two pregelatinized starch materials and two mixing speeds. It became clear that one mixer, the Ross PowerMix, was superior for the intended purpose when acetaminophen-starch systems were used. By its increased effect upon many of the response variables, the PowerMix appears to generate a more intense shearing action which results in a clearly superior product. On the basis of these considerations, it is logical to pursue the PowerMix further as a processor for pharmaceutical wet granulation, and not the Fielder, for the materials currently under investigation.

It may be stating the obvious to say that the amount of granulating fluid in a wet granulation process is an important variable. Based upon established mechanisms of agglomeration (Newitt and Conway Jones, 1958), much work in the field has since focused on predicting the strength of agglomerates (Rumpf, 1974), estimating liquid saturation levels (Kristensen, 1988) and estimating the liquid requirement for a given system (Leuenberger, 1982). These quantities depend upon many physical and physicochemical properties of the drug and the excipients used in a wet granulation process can influence the effects of liquid addition during the process, as well as in-process materials and the finished product (Shangraw, Wallace and Bowers 1981, Shirakura, et al. 1992, Lerk, Bolhuis and DeBoer 1974). Particle size, size distribution, particle shape, specific surface, polymorphic form, solubility and other properties can be important. Experimental investigations in Manuscript II, however, omitted granulation fluid levels as a factor, focusing instead on the differences between processors. Given the profound effects that the PowerMix had on the pharmaceutical materials under investigation, we may reasonably expect this mixer, moreso than the Fielder, will magnify the effects of varying 
the levels of granulating fluid during the process. The effects of various fluid levels can now be examined in detail in this experimental work.

Wet granulation processes begin and end with dry material. In the intervening steps, liquid is both added and removed. This drying procedure can be accomplished using traditional methods such as placing the material on trays into an oven until the moisture content reaches some predetermined level. Afterward, the material undergoes a sizing process, in this case, a milling step. This method has the advantage of allowing the fragile, moist granules to lie undisturbed until dry, preserving the particle size acquired at the endpoint of granulation. This method of drying also has disadvantages. Case-hardening impedes mass transfer. Migration of soluble ingredients to the surface of the granule during drying, such as dies or even the drug itself, may cause alterations in content uniformity.

Fluid-bed drying offers a substantial departure from static drying techniques. It offers rapid, uniform drying with high convection rates through vigorous particle motion. It reduces case hardening and migration of solutes and generally produces material of greater bulk volume and enhanced compressibility (Lieberman, Lachman and Schwartz 1990, p.93) On the other hand, interparticle friction may wear down granules. This experiment seeks to determine what, if any, effect the method of drying has on the quality of the granulations and tablets, as compared with the standard method of tray-drying used in the first experiment.

This experiment uses a two-level four-factor factorial design with one replicate. One powerful advantage of factorial designs is that they can be projected, augmenting existing designs with additional levels or factors (Montgomery 1991, p. 335). We take advantage of that fact here, utilizing half of the experimental treatments from Manuscript II (only those which used the PowerMix and lower fluid levels) with four more treatments which use high fluid levels. These eight wet granulations are subdivided prior to drying 
into two portions, each of which is subjected to either conventional tray drying or fluid-bed drying, for a total of sixteen trials.

The purpose of this experiment is to:

1) Examine in greater detail the differences between Starch 1500 and Starch 1551

2) Describe and quantify the effects of varying granulation fluid levels on in-process materials and end-stage products.

3) Quantify the effects of the high-shear functionality of the PowerMix in the presence of changing fluid levels.

4) Identify and quanify the effects of alternative drying methods on product quality 
EXPERIMENTAL

Materials

Starch 1500 (Colorcon, Inc., West Point, PA) and Starch 1551 (National Starch Lot\# FJ7343, Bridgewater, NJ) were obtained from Colorcon, Inc. Acetaminophen USP was obtained from Mallinckrodt (Lot\#4899-2K-768). Magnesium Stearate NF was obtained from Fisher Scientific (Lot\#742748, Fairlawn, NJ).

Formula

\begin{tabular}{|l|c|c|}
\hline Ingredient & $\mathrm{mg} / \mathrm{tab}$ & $\%$ \\
\hline Acetaminophen USP & 337.500 & 90.00 \\
\hline Pregelatinized Starch NF & 35.625 & 9.50 \\
\hline Purified Water USP & $*$ & $*$ \\
\hline Magnesium Stearate NF & 1.875 & 0.50 \\
\hline Total & 375.000 & 100.00 \\
\hline
\end{tabular}

* Water is expelled during processing 
METHODS

\section{Experimental Design}

This experiment is utilizes two levels of four factors arranged into a full factorial design with one replicate. The four main factors are the volume of water used (VOLUME), the speed at which the chopper is operated (SPEED), the type of starch (STARCH) and the method by which the granules are processed following wet granulation, that is, either traydrying with milling, or drying by fluid-bed drier (DRYING). Capitalized letters are used to designate each of the four factors in subsequent discussions.

Each of the four factors is used at two levels. Two factors are quantitative (VOLUME and SPEED), while the other two are qualitative (STARCH and DRIER). The speed of mixing refers to the chopper, which is set at either $19 \mathrm{~Hz}$ or $55 \mathrm{~Hz}$, corresponding to 1928 RPM and 3230 RPM, respectively. The planetary stirrer is held constant at $19 \mathrm{~Hz}$ (32 RPM). The volume of granulating fluid is varied between $280 \mathrm{ml}$ and $340 \mathrm{ml}$. The type of starch used in the process is either Starch 1500 or Starch 1551. Two methods of drying are tested. Tray-drying in a circulating-air oven is accompanied by a milling process; alternatively, the granules are dried in a fluid-bed drier.

The experimental design for this investigation is pictured in Figure 1. Statistical analyses were performed using SAS ${ }^{\mathrm{TM}}$ (SAS Institute, Cary, NC, Release 6.08). Backward elimination regression analyses use 0.10 as the criteria for retention in the model $($ SLSTAY=0.10). 
Figure 1

Experimental Design

(A 4-Factor 2-Level Full Factorial Design)

\begin{tabular}{|c|c|c|c|c|}
\hline & Starch Grade & Chopper Speed & Water Level & $\begin{array}{c}\text { Method of } \\
\text { Drying }\end{array}$ \\
\hline Batch \# & & $(\mathrm{Hz})$ & $(\mathrm{ml})$ & \\
\hline $97 \mathrm{~T}$ & 1500 & 19 & 280 & TRAY \\
\hline $93 \mathrm{~T}$ & 1500 & 19 & 340 & TRAY \\
\hline $99 \mathrm{~T}$ & 1500 & 55 & 280 & TRAY \\
\hline $96 \mathrm{~T}$ & 1500 & 55 & 340 & TRAY \\
\hline $100 \mathrm{~T}$ & 1551 & 19 & 280 & TRAY \\
\hline $98 \mathrm{~T}$ & 1551 & 19 & 340 & TRAY \\
\hline $95 \mathrm{~T}$ & 1551 & 55 & 280 & TRAY \\
\hline $92 \mathrm{~T}$ & 1551 & 55 & 340 & TRAY \\
\hline & & & & \\
\hline $97 \mathrm{FB}$ & 1500 & 19 & 280 & GLATT \\
\hline $93 \mathrm{FB}$ & 1500 & 19 & 340 & GLATT \\
\hline $99 \mathrm{FB}$ & 1500 & 55 & 280 & GLATT \\
\hline $96 \mathrm{FB}$ & 1500 & 55 & 340 & GLATT \\
\hline $100 \mathrm{FB}$ & 1551 & 19 & 280 & GLATT \\
\hline $98 \mathrm{FB}$ & 1551 & 19 & 340 & GLATT \\
\hline $95 \mathrm{FB}$ & 1551 & 55 & 280 & GLATT \\
\hline $92 \mathrm{FB}$ & 1551 & 55 & 340 & GLATT \\
\hline
\end{tabular}


Granulation

The Ross PowerMix \& Model PD-2 was made available from Charles Ross \& Son Co., Inc. Hauppauge, NY. Working capacity 1.5 gallons; planetary stirrer at $19 \mathrm{~Hz}$; highspeed disperser $19 \mathrm{~Hz}$ (low speed=1928 rpm) and $55 \mathrm{~Hz}$ (high speed=3230 rpm).

Liquid was added during granulation by pumping at $60 \mathrm{ml} / \mathrm{min}$ using a Masterflex Peristaltic Pump \#7553-30.

Tray-drying was done in a circulating hot air oven (Thelco Tray Oven Model 28). Fluid-bed drying was performed in a bench-top Glatt® drier (Glatt, Inc., Ramsey, NJ). Approximately 500 gram samples are dried until exhaust temperature reaches $60^{\circ} \mathrm{C}$.

Milling operations were performed using a Stokes Oscillating Mill through a \#12 wire mesh screen. Lubrication with Magnesium Stearate was performed in a Turbula Blender for two minutes.

Particle Size Testing

Sieve analyses were performed with approximately 20 -gram samples of dried granulate added to a stack of US Standard Sieves, dried and tared. Sieving was done using a Van-Kel Model 18480 Sieve Shaker for 5 minutes at a \#7 setting, or until no further change takes place. Analyses are performed in triplicate. The geometric mean, volumesurface mean sizes and geometric standard deviation are calculated directly as described in Manuscript I. 


\section{Bulk Density}

Free-flowing and tapped densities were determined by pouring $100 \mathrm{ml}$ samples through a funnel into a graduated cylinder, then tapped 1000 times. Volume determinations before and after tapping ( $v$ and $v_{0}$, respectively) a known weight of granulate are used to calculate the Carr (1965) Compressibility Index, expressed as a percentage:

$$
\text { Compressibility Index }=\left[1-\frac{v}{v_{0}}\right] \cdot 100
$$

Angle of Repose

Dried unlubricated granules are poured through a funnel to a known height. The diameter of the cone-shaped pile is measured. The angle is the degrees of elevation from the horizontal, as determined by the following formula:

$$
\text { Angle of Repose }=\arctan \left(\frac{\text { height }}{\text { radius }}\right)
$$

Tableting

Tablets were compressed using an instrumented rotary tablet press (PennwaltStokes Model 900-512) operating at $30 \mathrm{rpm}( \pm 1)$ adjusted to three compression settings approximating $12 \mathrm{kN}, 16 \mathrm{kN}$ and $20 \mathrm{kN}$. The tablets are compressed using a single punch and die set (7/16-inch round, flat-faced, no bevel-edge) to a weight of $375 \mathrm{mg}$. During 
tableting, a minimum of thirty tablets are compressed. Each compression-ejection cycle is measured and recorded individually; means and standard deviations of each of the following parameters are generated by the data acquisition and analysis software:
1) Peak Compression Force
$(\mathrm{kN})$
2) Area Under Compression Force-Time Curve
$(\mathrm{kN} \cdot \mathrm{msec})$
3) Peak Ejection Force
4) Area Under Ejection Force-Time Curve
$(\mathrm{N} \cdot \mathrm{msec})$

The tablet 'cohesion index' is calculated from the following formula:

$$
\text { Cohesion Index }=\frac{\text { Crushing Force }(\mathrm{kg})}{\text { Peak Compression Force }(\mathrm{kN})} \times 10^{5}
$$

\section{Tablet Weight}

At least ten tablets are weighed indiviually on a Mettler AE 240 Digital Balance to an accuracy of \pm 0.00001 grams. The mean, standard deviation and relative standard deviations are determined.

\section{Tablet Thickness}

The thickness of at least ten individual tablets is measured using an analog micrometer (Mitutoyo Analog Micrometer) accurate to \pm 0.0001 inches. 
Tablet Hardness

The diametral crushing strength of at least ten individual tablets is measured using the Erweka Tablet Hardness Tester Model TBT. Results are reported in kilograms.

Tablet Friability

Twenty tablets of known weight are allowed to fall from a height of six inches inside a baffled chamber revolving at 25 RPM for four minutes. The tablets are dedusted and reweighed. Friability is the loss in weight, expressed as a percentage.

Tablet Disintegration Time

The disintegration time of six individual tablets is measured using the USP Disintegration Apparatus, with water at $37^{\circ} \mathrm{C}$ as the medium. 
RESULTS

\section{Analysis of Granulations}

\section{Granulation Particle Size}

The median, geometric mean and surface-volume mean diameters for all finished granulations were evaluated. Regression analysis of the particle size data shows that, of the three sizing parameters, the volume-surface mean diameter $d_{v s}$ can most closely be fitted with a linear model. A backward elimination procedure, beginning with all main factors and interactions, results in the following model $\left(r^{2}=0.93\right)$ :

$$
d_{v s}=477-68.0 \bullet S p d+22.2 \bullet V+26.7 \bullet D+29.7 \bullet S p d \bullet V
$$

where Spd=speed of mixing (rpm); V=volume of water added; $D=$ method of drying (tray or fluid-bed); Stch=type of starch

All terms left in the model were significant at the $99 \%$ level or greater. The effects of SPEED, VOLUME and the SPEED•VOLUME interaction can be seen in Figure 2, where particle size decreases with increases in mixing speed (sloping down toward the front) at a rate threefold greater than it increases with fluid levels. The interaction effect is depicted by the relatively smaller decrease in particle size with mixing speed when higher water levels are used, e.g. the surface does not slope downward to the same degree and some surface curvature results.

There were no significant differences in any of the size parameters attributable to the type of STARCH. 
The method of DRYING had a significant effect on the surface-volume mean particle size (but not the other particle parameters). Tray-drying with milling resulted in values of $d_{\nu s} 12 \%$ smaller, on average, than fluid-bed drying.

Granulation Size Uniformity

Particle size uniformity was almost entirely a function of mixing SPEED $(p<0.0001)$. Figure 3 shows a plane declining steeply as mixing speed increases, with little or no curvature and whose level curves remain essentially parallel to the axis for water levels. This clearly indicates improved granulation uniformity with higher mixing speeds, as well as the insignificance of different water levels $(p=0.43)$.

Tray-drying/milling resulted in only slightly (7\%) improved particle size uniformity, and this difference was significant $(p=0.03)$. The type of STARCH had no effect, and there were no interaction effects. In summary, by far the most important factor in producing more uniform granulations is higher mixing speeds. 
Figure 2

\section{Granule Volume-Surface Mean Diameter}

(microns)

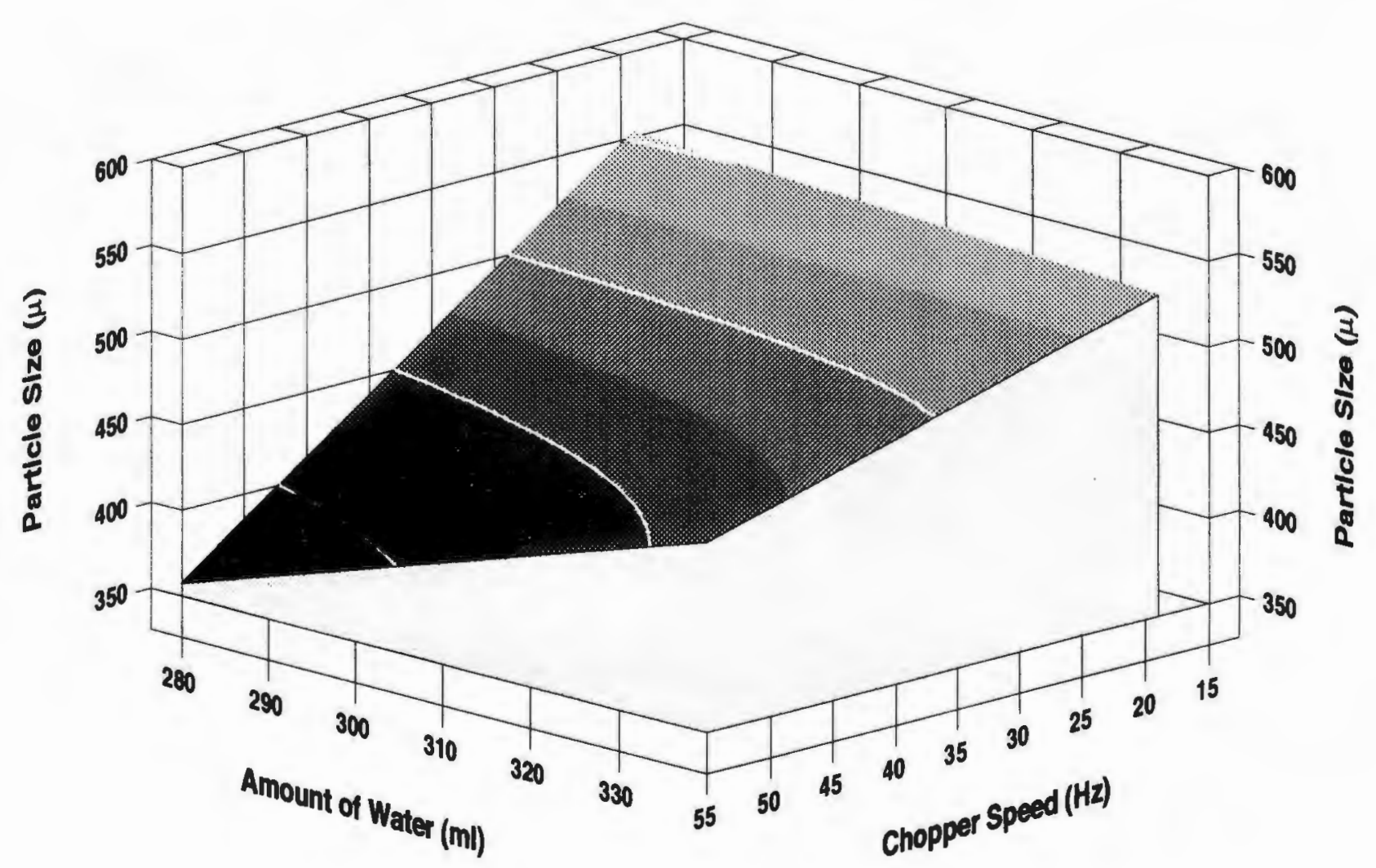


Figure 3

Standard Deviation of the Geometric Mean Particle Size

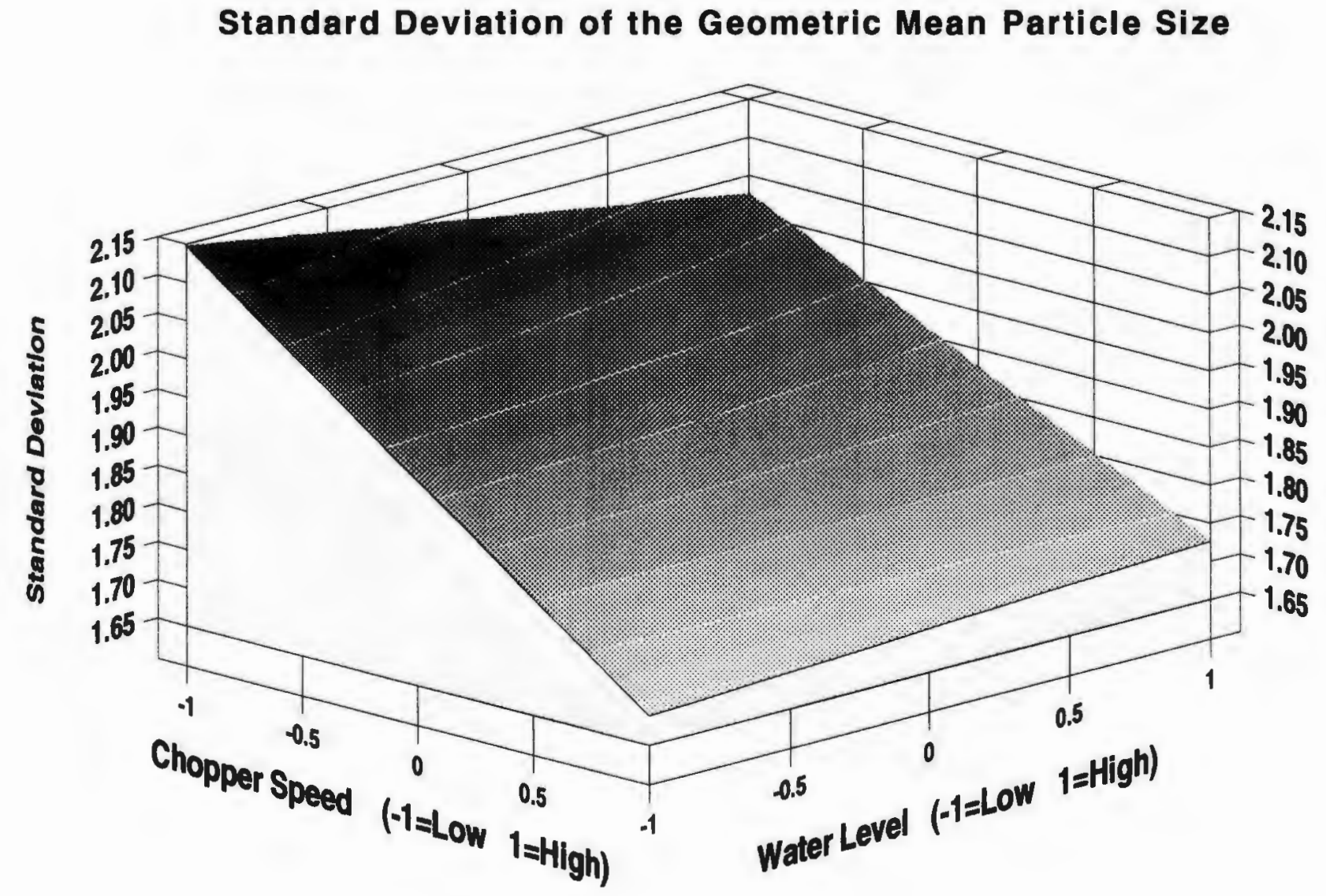


Flow Analysis: Carr Compressibility Index

Backward regression analysis results show evidence that only two main factors significantly affect flowability as measured by the Carr Compressibility Index. The SPEED of mixing ( $p=0.001)$ and the VOLUME of water used $(p=0.0002)$. Among twofactor interactions, only the STARCH•SPEED interaction was significant $(p=0.0015)$.

Using a greater VOLUME of water $(340 \mathrm{ml})$ caused the most improvement in flowability $(22 \%)$. This supports the our premise that this formulation benefits significantly from a wet granulation process. The type of STARCH used had only a small and marginally significant effect $(p=0.05)$ on the Carr compressibility index. Indices for Starch 1500 were $10 \%$ lower, on average, suggesting the potential for better flow characteristics overall, although a single batch (92-FB) using Starch 1551 at high speed and high water levels produced the single best results $(12.7 \%)$. When the speed of mixing and the water level were lowered (Batch 100-Tray), the worst individual results were obtained $(27.3 \%)$.

The effects of mixing SPEED differed significantly for the two starches. Figure 4 shows the interaction between STARCH and SPEED $(p=0.0015)$. Mixing speed had no effect on the compressibility of Starch 1500 granulations, but compressibility decreased substantially for Starch 1551 with higher speeds, causing a $29 \%$ improvement, on average. This may indicate that the Starch 1551 may be 'activated' at higher factor levels, whereas Starch 1500 seems unaffected. In a less granulated state, then, Starch 1500 exhibits the potential for better flow, but with increasing speeds the difference between starches can be overcome. The method of DRYING had a small but insignificant effect $(p=0.11)$; tray drying yielded slightly larger (worse) values. 
Figure 4

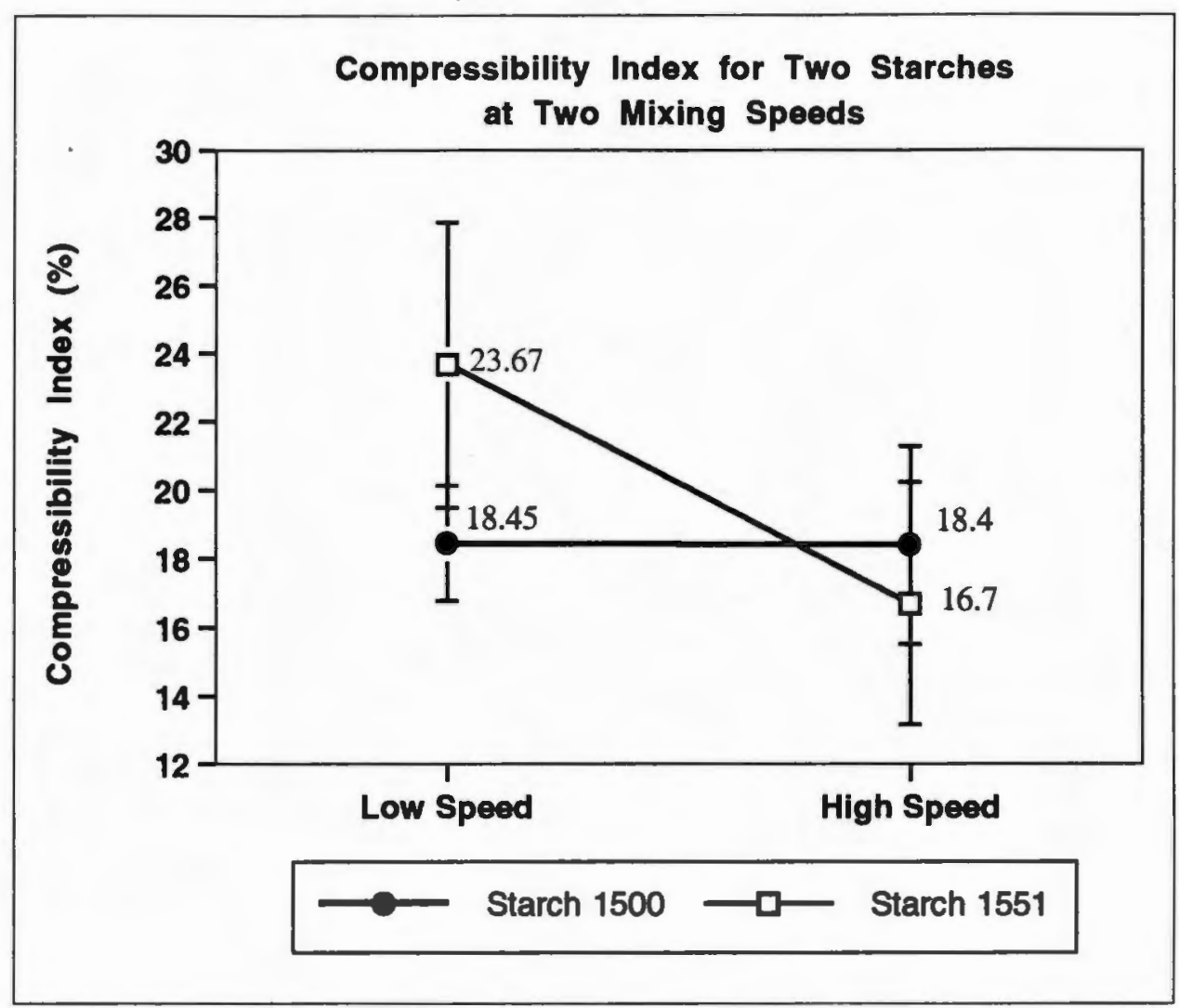


Flow Analysis: Angle of Repose

Granulation flowability was a function of the type of STARCH $(p=0.0008)$, the VOLUME of water $(p=0.0004)$ and the SPEED of mixing $(p=0.01)$. The interaction effect between STARCH and VOLUME OF WATER was also significant $(p=0.001)$.

Starch 1500 yielded better-flowing granulations than Starch 1551, which supports the results obtained using the Carr Index. The mean decrease in the angle of repose was 1.03 degrees. Higher mixing speeds caused a smaller improvement ( 0.7 degrees). Using $340 \mathrm{ml}$ of water instead of $280 \mathrm{ml}$ caused the greatest improvement (1.11 degrees), a result which is also supported by compressibility testing.

Figure 5 shows the interaction between STARCH and VOLUME of water. Starch 1500 was lower overall, but Starch 1551 showed a more dramatic improvement in flowability with the addition of more water. The vertical separation between lines for Starch 1551 illustrates this.

Mixing speed had little effect upon Starch 1551, as well as Starch 1500 at low water levels. Starch 1500 benefited greatly from higher speeds when more water was used, however, showing a mean decrease of 1.28 degrees.

As measured by both the Carr compressibility index and angle of repose, then, flowability was a strong function of both SPEED of mixing and WATER levels. In both cases, flowability improved when more water and higher mixing speeds were used. Water was more important than mixing speed in both cases. The effect of STARCH type had a significant effect using angle of repose as a measure of flowability, but was only marginally important using the compressibility index. The method of DRYING had no effect in either case. 
Table 1

Flowability Results: Angle of Repose and Compressibility Indices

\begin{tabular}{|c|c|c|c|c|c|c|}
\hline Batch \# & \multicolumn{3}{|c|}{ Angle of Repose (degrees) } & $\begin{array}{c}\text { Loose } \\
\text { Density } \\
\text { (g/ml) }\end{array}$ & $\begin{array}{l}\text { Tapped } \\
\text { Density } \\
(\mathrm{g} / \mathrm{ml})\end{array}$ & $\begin{array}{c}\text { Carr } \\
\text { Index } \\
(\%)\end{array}$ \\
\hline 92 Tray & 36.07 & 35.94 & 35.68 & 0.467 & 0.549 & 14.9 \\
\hline $92 \mathrm{FB}$ & 36.62 & 35.84 & 35.11 & 0.528 & 0.605 & 12.7 \\
\hline 93 Tray & 36.60 & 37.39 & 37.00 & 0.472 & 0.584 & 19.2 \\
\hline $93 \mathrm{FB}$ & 36.54 & 37.95 & 35.78 & 0.487 & 0.582 & 16.3 \\
\hline 95 Tray & 38.33 & 37.53 & 36.81 & 0.496 & 0.511 & 18.8 \\
\hline 95 FB & 38.66 & 37.30 & 37.36 & 0.504 & 0.633 & 20.4 \\
\hline 96 Tray & 34.01 & 33.69 & 33.85 & 0.451 & 0.545 & 17.2 \\
\hline $96 \mathrm{FB}$ & 35.71 & 36.69 & 34.75 & 0.505 & 0.595 & 15.1 \\
\hline 97 Tray & 35.89 & 35.71 & 36.33 & 0.491 & 0.615 & 20.2 \\
\hline $97 \mathrm{FB}$ & 37.05 & 36.32 & 35.04 & 0.534 & 0.652 & 18.1 \\
\hline 98 Tray & 35.85 & 34.35 & 35.89 & 0.502 & 0.634 & 20.8 \\
\hline $98 \mathrm{FB}$ & 35.78 & 37.35 & 36.21 & 0.516 & 0.635 & 19.4 \\
\hline 99 Tray & 37.69 & 34.02 & 35.19 & 0.457 & 0.584 & 21.7 \\
\hline $99 \mathrm{FB}$ & 37.23 & 34.52 & 36.57 & 0.485 & 0.603 & 19.6 \\
\hline 100 Tray & 38.56 & 37.98 & 37.42 & 0.490 & 0.674 & 27.3 \\
\hline $100 \mathrm{FB}$ & 37.82 & 39.27 & 38.56 & 0.503 & 0.691 & 27.2 \\
\hline
\end{tabular}


Figure 5

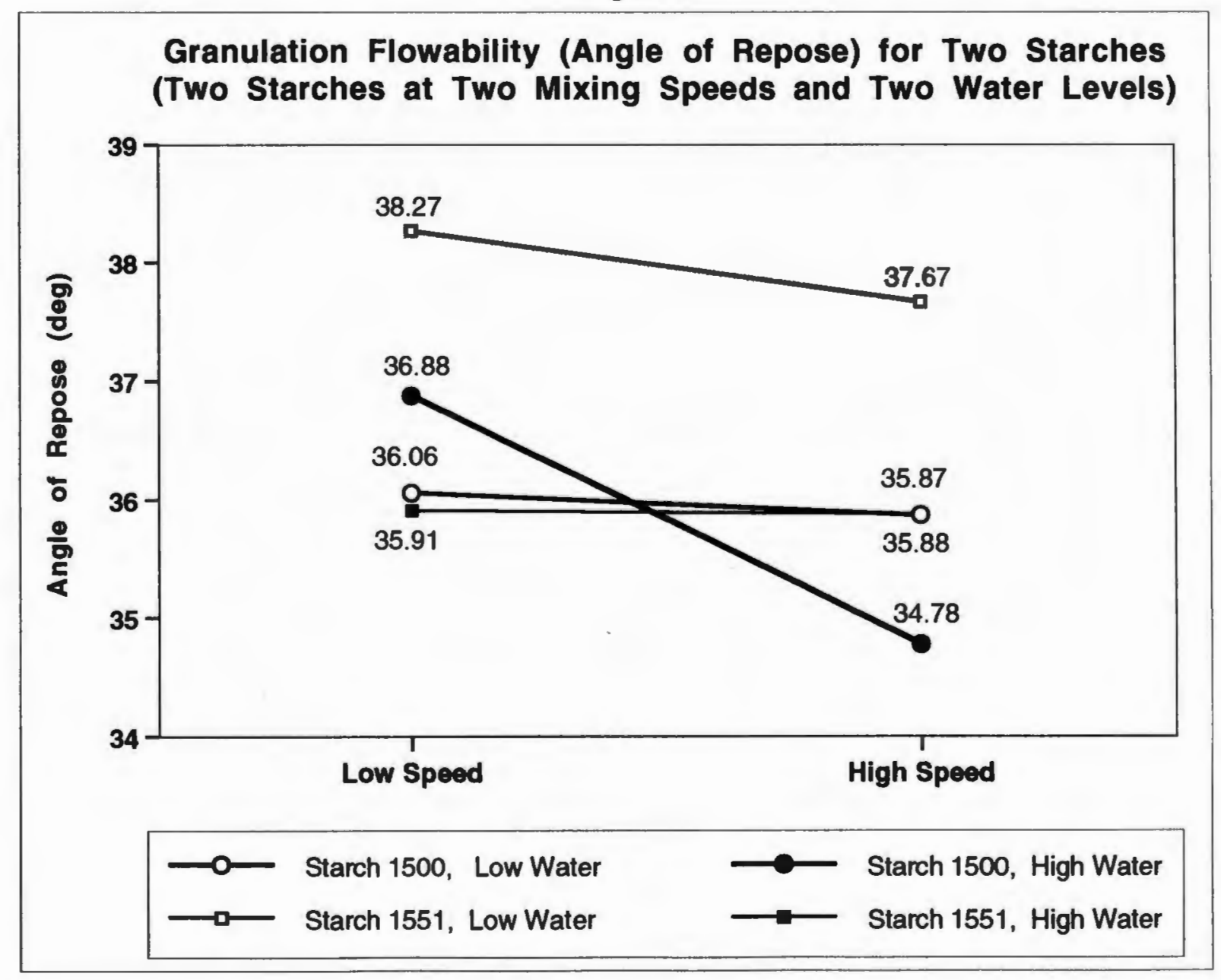




\section{Tableting}

Crushing Strength.

Tablets were compressed at approximately $12 \mathrm{kN}$. Diametral crushing strengths for each tablet were measured, then normalized by corresponding compressional forces. This calculated quantity, the 'cohesion index' (Veesler, 1992) is used as an index of compactability.

Mixing SPEED had the greatest effect on tablet strength $(p<0.0001)$, with higher speeds causing a marked (37\%) improvement in the cohesion index. The amount of WATER had a slightly lesser effect, causing a $29 \%$ improvement in the index $(p<0.0001)$. STARCH grade had a significant effect upon tablet strength. Granulations made with Starch 1500 were significantly more compressible $(p<0.0001)$. Tablets were $20 \%$ harder using Starch 1500 , on average.

These effects, as well as the difference between starches, can be seen in Figure 6, which illustrates the significant three-way interaction between these major factors $(p<0.0001)$. In three of the four cases higher mixing speeds increased hardnesses, particularly for Starch 1551 , which showed the greatest improvement. If more water is used (top line), Starch 1500 shows no improvement with increasing mixing speed. In the other three cases, however, the positive effect of higher mixing speed is evident.

The effect of the DRYING method on this parameter is significant $(p=0.02)$, but much smaller than the other three main factors. The cohesion index for tablets made from fluid-bed dried material is only $4.5 \%$ higher than from tray-dried material. 
Figure 6

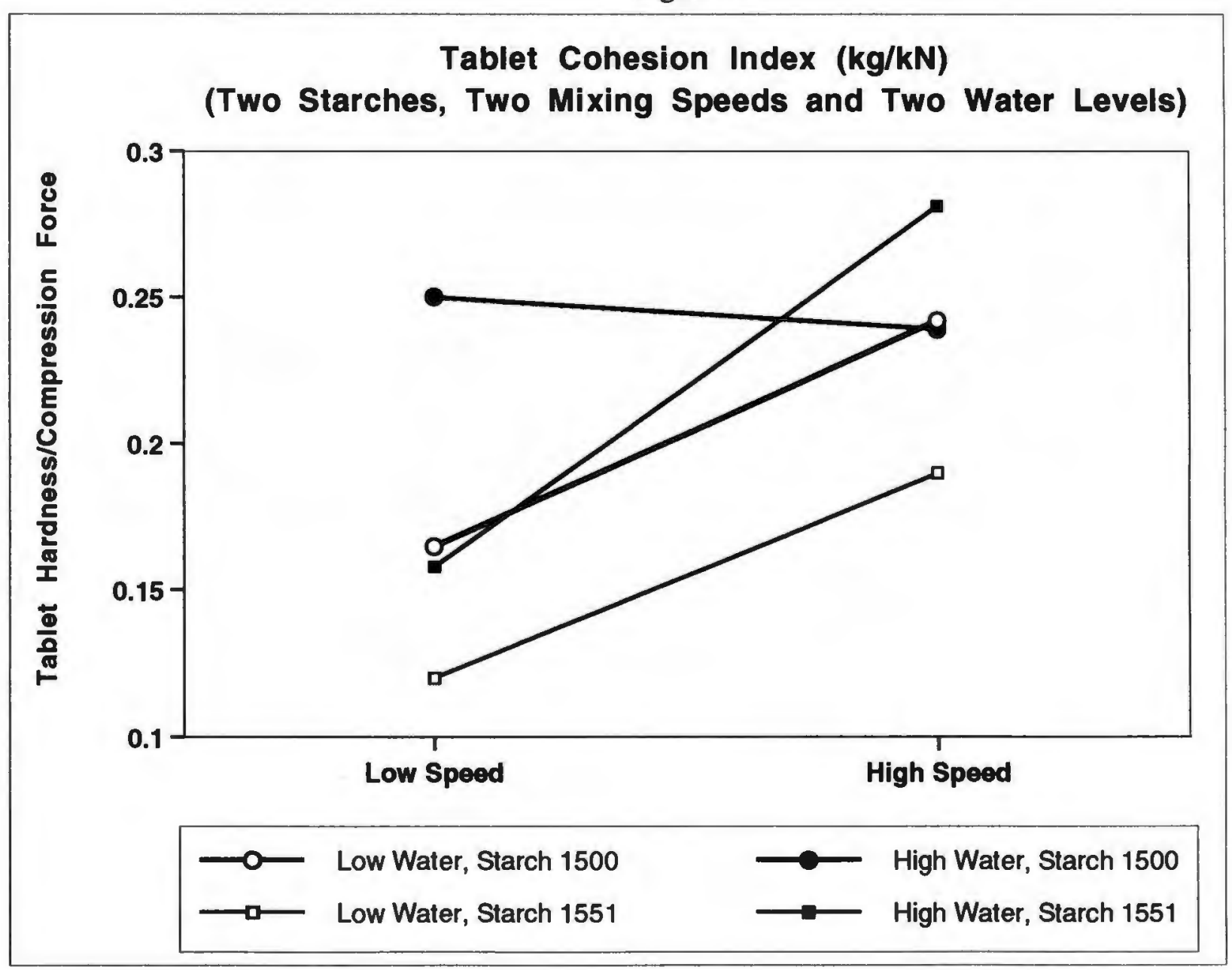




\section{Ejection Force}

All four main effects had a significant influence upon ejection force. Tablets made with Starch 1500 required $14 \%$ more ejection force during tableting $(p=0.003)$. This may be related to differences in tablet hardness, with harder tablets requiring more force to be ejected from their dies, as seen in Figure 7.

Among all main and interaction effects, mixing SPEED had the greatest influence $(p=0.001)$. The VOLUME of water used had a lesser effect $(p=0.028)$, as did the method of DRYING $(p=0.025)$.

Figure 8 shows the effects of mixing speed and water levels. For both Starch 1500 and 1551, higher WATER levels resulted in higher ejection forces (note the vertical separation). In all four instances, increases in mixing SPEED resulted in higher average ejection forces. The parallel nature of the increases indicates a lack of interaction effects.

The drier used had only a marginally significant effect $(p=0.025)$, with tray-dried granulations requiring slightly more ejection force. 
Figure 7

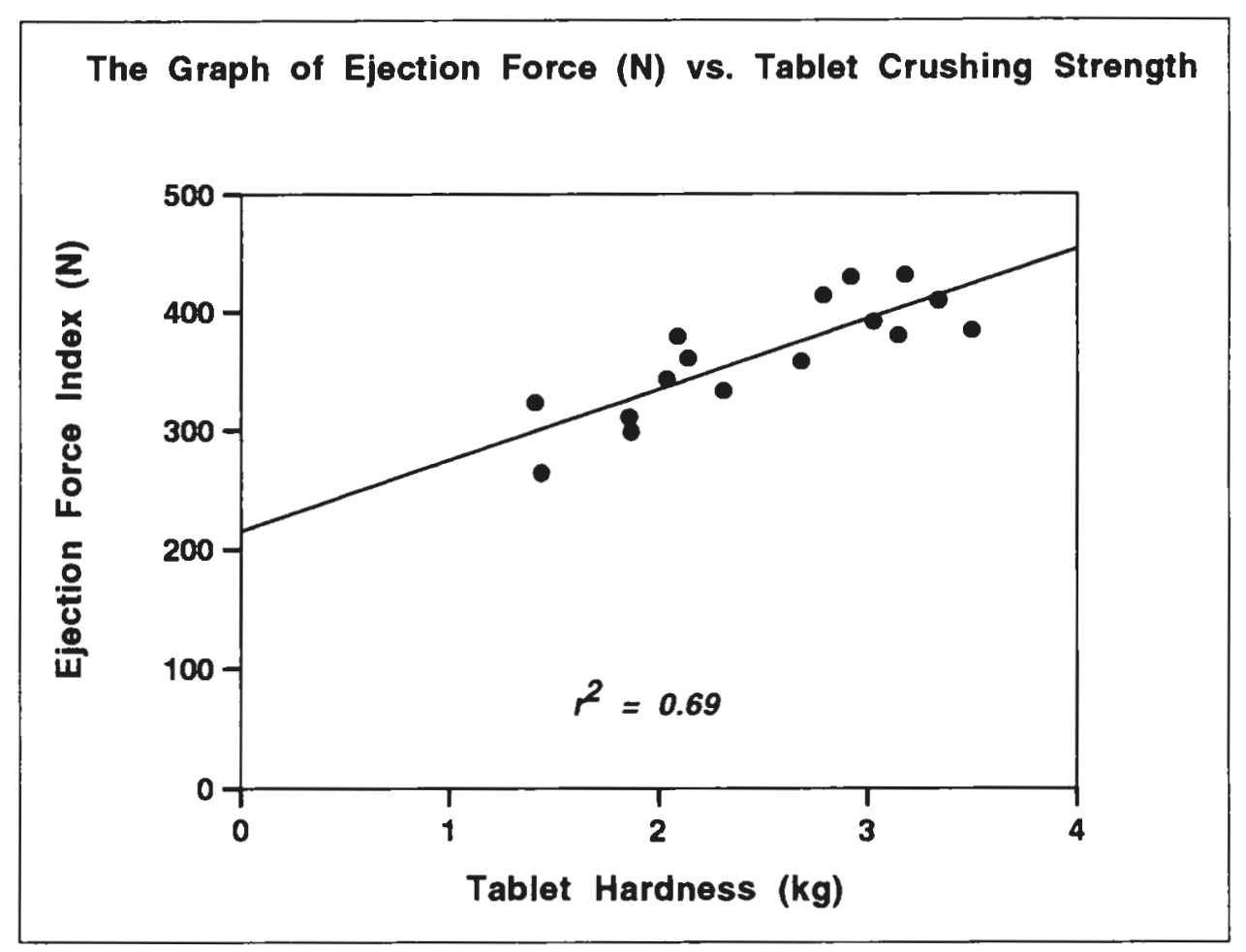


Figure 8

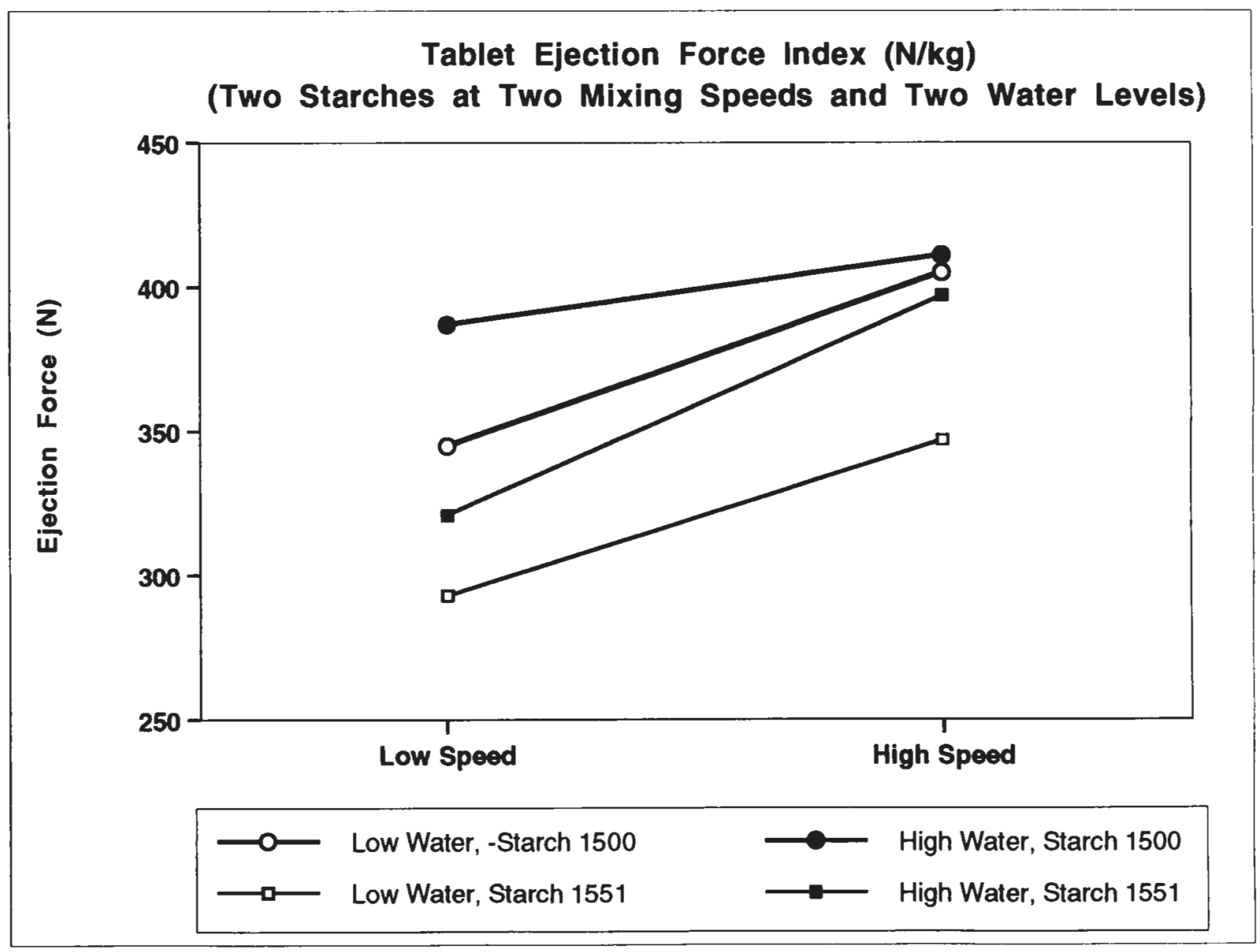


Weight Uniformity.

Tablet weight uniformity was evaluated both by direct measurement, and by the variability in peak compression forces. In both cases, there were virtually no differences attributable to the type of STARCH used. This is not surprising, since there were no significant differences in particle size or particle size distribution to cause variability in die filling.

High mixing SPEEDS made granulations that cut tablet weight variation approximately in half, from $0.61 \%$ to $0.34 \%$, which was statistically significant $(p=0.02)$. This was supported by an evaluation of the variability in peak compression forces, which also exhibited a significant reduction $(p=0.014)$. At low mixing speeds the compression peak RSD was $0.717 \%$, while high speeds resulted in tablets with a mean RSD of $0.358 \%$.

Increased amounts of WATER used in the granulation process reduced tablet weight variation and the variability in compression peak force approximately $38 \%$, but these differences were not statistically significant at the $95 \%$ level. There is a significant interaction $(p=0.04)$ between mixing speed and water level, however, seen in Figure 9. High mixing speeds lead to no improvement, but low mixing speeds benefited significantly from the additional granulating fluid.

The method of drying/milling had a negligible effect upon compression force variability, but was the most important factor in reducing tablet weight variation $(p=0.01)$. Tray-drying, followed by milling, caused a $50 \%$ reduction in the tablet weight RSD, compared with a $45 \%$ reduction caused by increased mixing speeds. 
Figure 9

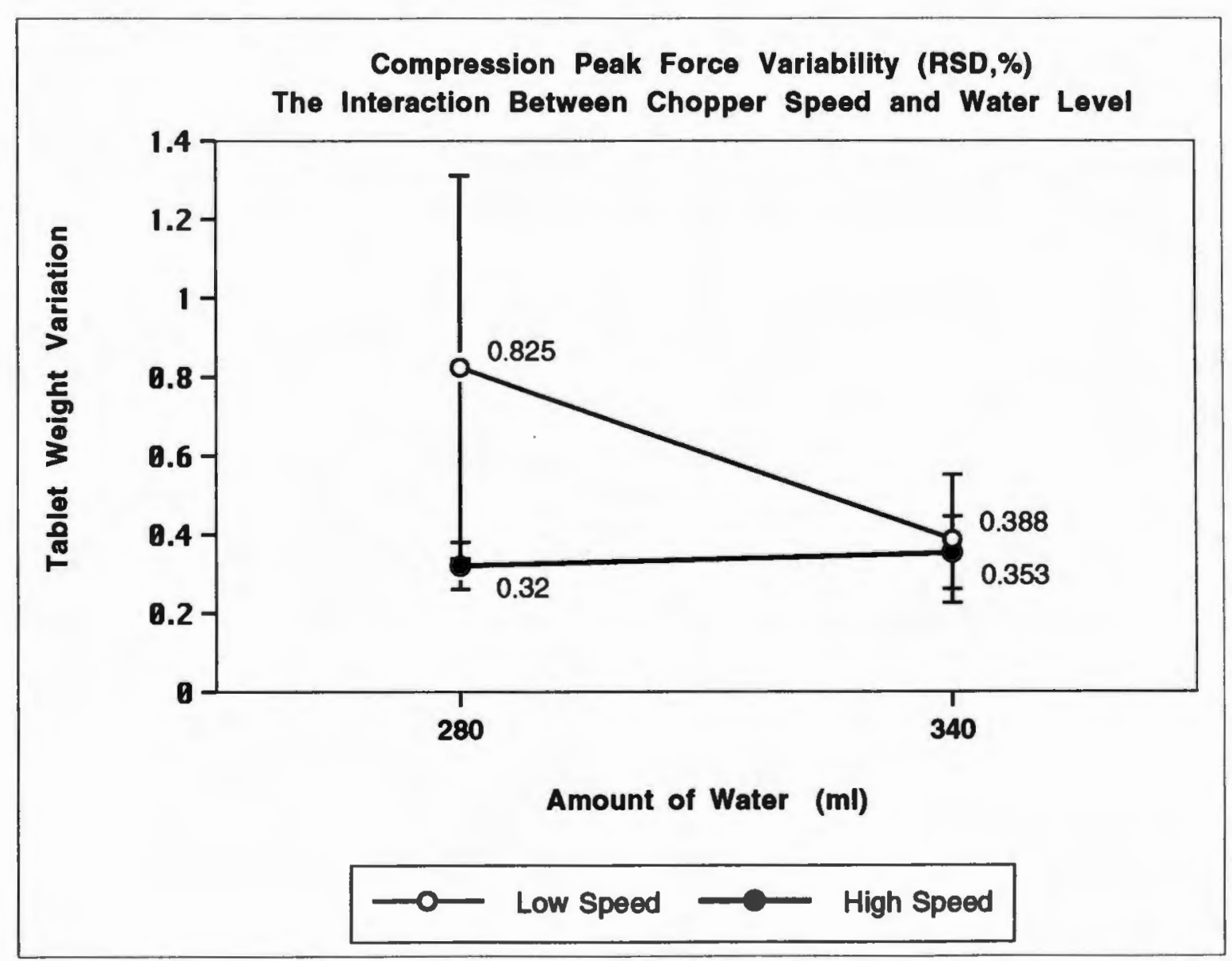


Friability.

Tablet friabilities for all batches were high. Without exception, all exceeded the generally accepted limit of 0.8-1.0\% (Lachman and Lieberman 1986, p. 88). Values ranged, however, from just over $1 \%$ to a maximum of $17 \%$. Starch 1500 made tablets whose friabilities were significantly lower than those made with Starch $1551(p=0.03)$, on average. Mean friabilities were $5.9 \%$ with Starch 1500 and $8.0 \%$ with Starch 1551 . The best tablets from any one combination of factors had a friability of $1.1 \%$; these were made with Starch 1551 . These were followed by friabilities of $1.3 \%$ and $1.4 \%$, made with Starch 1500.

Higher levels of both mixing speed and water level caused a marked improvement in tablet durability $(p<0.0001$ and $p=0.0003$, respectively). Figure 10 shows the decrease in friability from nearly $8 \%$ to almost $1 \%$ as both factors increase together from their lowest to their highest levels. 
Figure 10

Tablet Friability (\% Abrasion)

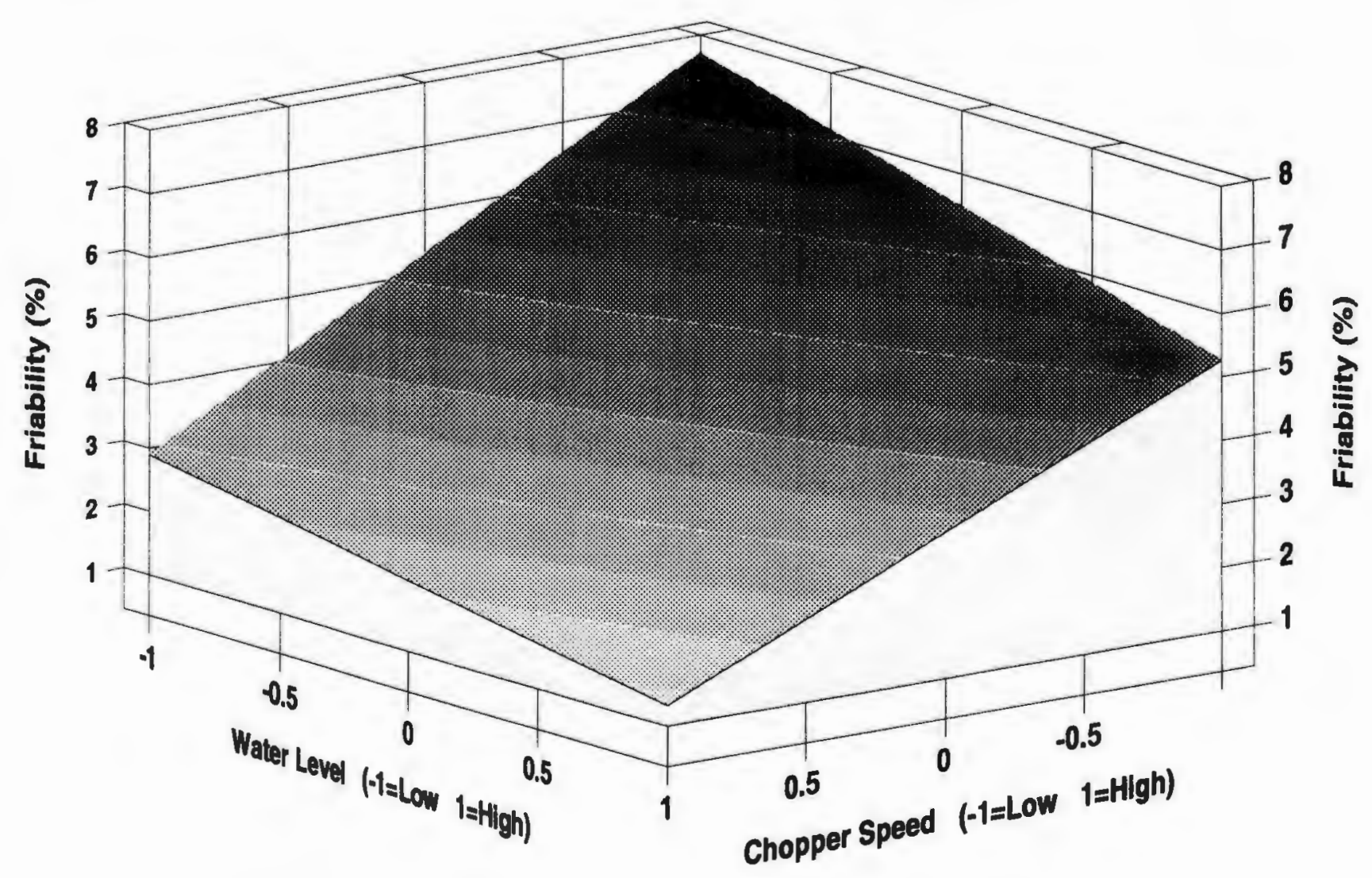




\section{Disintegration Time}

Tablet disintegration times were short, with all tablets disappearing within 2.5 minutes. Nevertheless, there were large differences due to mixing SPEED $(p<0.0001)$, the VOLUME of water added $(p<0.0001)$, the interaction between them $(p<0.0001)$, as well as a three-way interaction between STARCH, SPEED and WATER $(p<0.0001)$. High-speed mixing increased disintegration time $230 \%$, increasing the water level caused a $100 \%$ increase, fluid-bed drying caused an $11 \%$ increase, and Starch 1500 caused a $6 \%$ increase.

Figure 11 shows how disintegration times increase for all four combinations of starch and water levels as mixing speed is increased. When low water levels are used, Starch 1500 tablets disintegrate more slowly than Starch 1551 at low speeds, and disproportionately more slowly at high speeds. Apparently, Starch 1500 has a greater ability as a binder when smaller amounts of water are used.

The situation reverses when more water is used. Consider the results for Starch 1500 at both high and low water levels. The lines are parallel, meaning the response of the starch to changes in mixing speed is the same. Starch 1551 , on the other hand, which causes a dramatic increase in disintegration time at higher mixing speeds, but only when more water is used. In some sense, then, Starch 1551 is 'activated' more strongly by water than Starch 1500. 
Figure 11

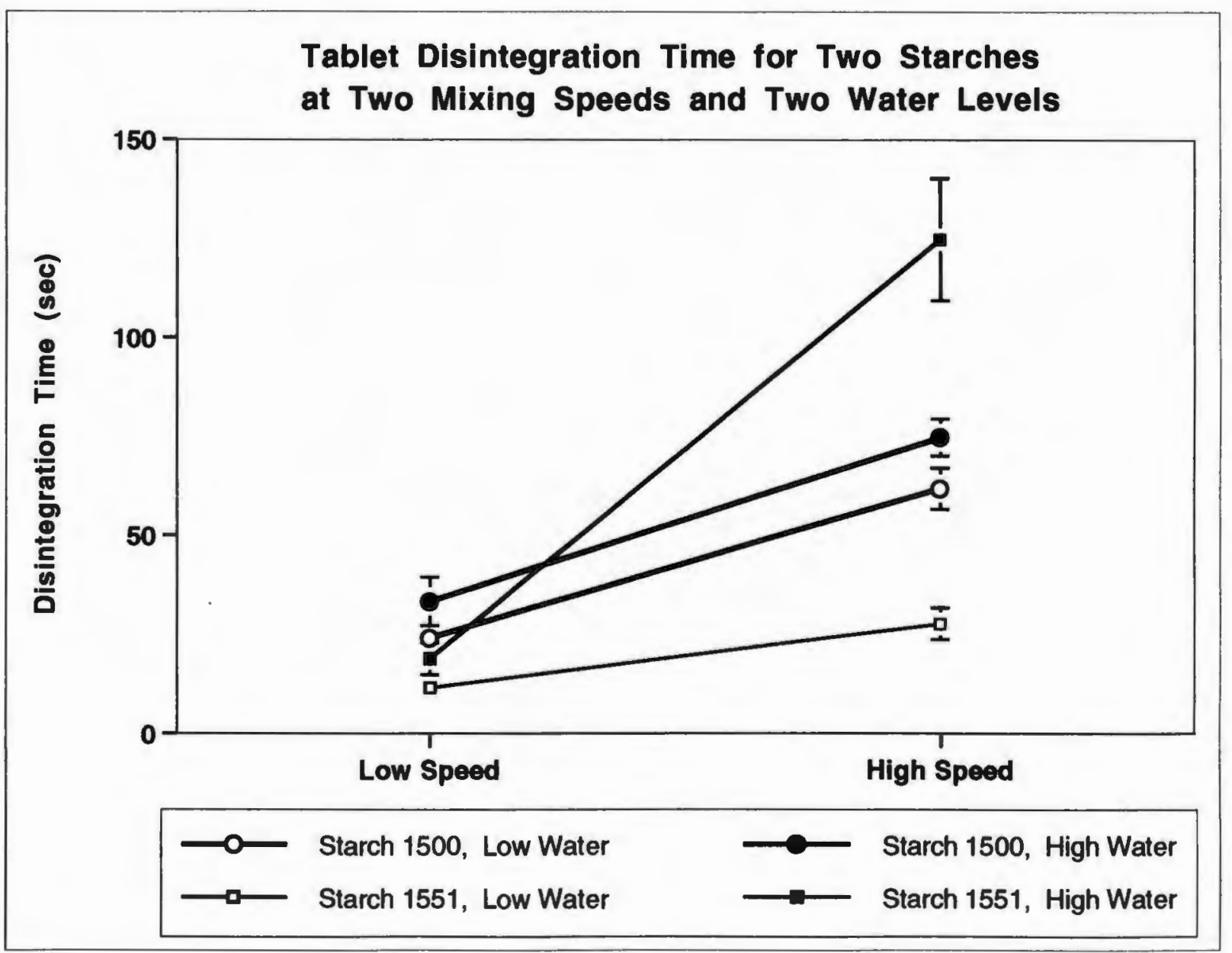


DISCUSSION

Nine response variables are evaluated for an experiment consisting of four main factors. One factor is a formulation variable; the remaining three are process variables. These four variables, two of which are quantitative and two qualitative, are assembled into a full factorial design experiment. This allows the effects of each main factor, as well as their interactions, to be estimated.

The nine response variables consisted of measurements of the physical properties of granulations and tablets made from them, as well as indirect measurements of the forces exerted during the compaction process. This collection of responses constitutes a rather broad and detailed assessment of granulation quality.

Of these four experimental variables, the speed of mixing had the most profound effects on the responses measured. All were significantly affected when the speed of mixing was increased. Most importantly, it enhanced the essential properties of the pharmaceutical materials under study, that is, the uniformity, flow, compressibility, compactability and durability of the granulations and tablets. Only two, ejection force and tablet disintegration time, were increased where lower values are usually the desired outcome. Even so, these two parameters indicate an increase in tablet strength, and therefore serve to fortify the conclusion that the intense mechanical action of this mixer during the course of a wet granulation process produces substantial benefit.

Liquid added during a wet granulation process serves as a catalyst for the creation and maintenance of interparticulate bonds. Through the intervention of water or some or other fluid, powder beds are transformed in terms of size and shape, and possibly other physical and physicochemical properties. These changes, in turn, should enhance their suitability for a particular purpose, such as a tableting process. 
This experiment contrasts the effects of using two different levels of water addition. All but one of nine parameters exhibited significant differences attributable to this factor. In nearly every case where a particular improvement is sought, increasing the amount of water used in the process has the desired effect. Like increases in mixing speed, however, ejection force and tablet disintegration time increased with water level. These parameters help to substantiate the evidence that the resulting granulations form stronger compacts. The lone case which shows no significant alteration with water addition is granulation uniformity, which was almost entirely a function of speed. Within the experimental range of 280 to 340 milliliters of water used, the intensity of mixing was adequate to uniformly distribute the liquid throughout the powder bed and subsequently control particle size, so that any substantial change in particle size distribution is likely overcome by shear.

There is no evidence to support the contention that Starch 1500 is inferior to Starch 1551 when subjected to a high-shear granulation process. Only half of the responses displayed statistically significant differences. between starches. With the exception of granulation flowability, all the differences were related to tablet compactability and strength. In each case, Starch 1500 was superior. Granulation flowability, as measured by the Carr Compressibility Index and the angle of repose methods, was better for Starch 1500 granulations.

Few of the parameters measured showed any sensitivity to the method of drying the granules. The average particle size of the tray-dried and milled granules was smaller and very slightly more uniform, as might be expected from any milling process. Tablets compressed from relatively intact, larger-sized granules may be expected to disintegrate somewhat more slowly, since individual granules must disintegrate as well, not just the 
tablet proper. In all, it appears that the method of drying has limited impact upon the performance of these granulations. Their properties are essentially determined by the time they reach the drying stage in the process. 


\section{CONCLUSIONS}

The acetaminophen-starch formulation under study clearly required an aggressive level of granulation processing in order to meet even minimal requirements for tableting. Despite frequently substandard results as a dosage form, important differences between levels of four factors were delineated. High mixing speeds and higher water levels were instrumental in improving nearly all performance parameters tested. To a much lesser extent, Starch 1500 was preferable over Starch 1551, while the method of drying had little effect. 
REFERENCES

Carr R.L. (1965) Evaluating Flow Properties of Solids, Chemical Engineering, 72, 163168.

Kristensen H.G. (1988) Agglomeration of Powders, Acta Pharmaceutica Suecica, 25, 187204.

Lerk I.C.F., Bolhuis G.K.De Boer A.H. (1974) Comparative Evaluation of Excipients for Direct Compression, Pharmaceutisch Weekblad, 40, 945-955.

Leuenberger H. (1982) Granulation, New Techniques, Pharmaceutica Acta Helvetica, 57(3), 72-81.

Lieberman H.A.,Lachman L., Schwartz J.B. (1990) Pharmaceutical Dosage forms: Tablets, Volume 2, Second Edition, Marcel Dekker, New York.

Montgomery D.C., Design and Analysis of Experiments (1991) Design and Analysis of Experiments, Third Edition, John Wiley \& Sons, New York.

Newitt D.M., Conway-Jones J.M. (1958), Transactions of the Institution of Chemical Engineers, 36, 422.

Rumpf H. (1974), Chemical . Ingineering. Technologie., 46, 1. 
Shangraw R.F., Wallace J.W., Bowers F.M. (1981) Morphology and Functionality in Tablet Excipients for Direct Compression: Part I, Pharmaceutical Technology, 10, 1-10.

Shangraw R.F., Wallace J.W., Bowers F.M. (1981) Morphology and Functionality in Tablet Excipients for Direct Compression: Part II, Pharmaceutical Technology, 10, 11-19.

Shirakura O.,Yamada M., Hashimoto M., Ishimaru S., Takayama K., Nagai T. (1992) Effect of Amount and Composition of Granulating Solution on Physical Characteristics of Tablets, Drug Development and Industrial Pharmacy, 18(10) 1099-1110.

Veesler S., Boistelle R., Delacourte A., Guyot J.C., Guyot-Hermann A.M. (1992) Influence of Structure and Size of Crystalline Aggregates on Their Compression Ability, Drug Development and Industrial Pharmacy, 18(5), 539-560. 


\section{Manuscript IV}

The Spheronization of Theophylline-Microcrystalline Cellulose Combinations

Using a Fluidized Bed Rotogranulation Technique 


\section{ABSTRACT}

A fluidized-bed apparatus for the agglomeration and spheronization of pharmaceutical powders was used to test the suitability of a well-known spheronizing agent, microcrystalline cellulose (MCC), in a process using anhydrous theophylline as a model drug. Three available grades of theophylline and microcrystalline cellulose, differing chiefly in particle size and shape, were used together in all permutations to isolate any differences between them. The study found that in concentrations of below $50 \%$ drug, all combinations of drug and excipient performed well, and resulted in spherical pellets whose properties were largely indistinguishable. It was only possible to make spheres in all concentrations up to $90 \%$ using the largest-sized grade of theophylline (designated as Type A, or as K-205). The finest, micronized grade of theophylline (Type C) could only be spheronized at drug loading levels not exceeding $50 \%$.

The spheres were characterized as to size, shape, uniformity of size and shape, density, friability, sphericity and drug content. Among MCC grades, only drug content differed slightly, with the smallest particle-size MCC (Avicel H-101) resulting in slightly higher potency pellets. It was theorized that this potency difference resulted from a loss of MCC duirng fluidization, increasing the proportion of drug left in the spheres.

A second experiment used only the coarsest grade of theophylline (Type A) with the three grades of MCC to detect changes in pellet roundness as a function of drug content. Drug loading levels of $25 \%, 50 \%, 70 \%$ and $90 \%$ were used in a two-factor ANOVA design. Results showed that pellets of concentrations exceeding $70 \%$ suffered a decrease in pellet roundness.

In all, it was found that the potential for spheronization of binary systems using anhydrous theophylline and microcrystalline cellulose depended primarily on the grade of theophylline chosen, and to a much lesser extent, the grade of MCC. 


\section{INTRODUCTION}

The concept of multiple unit formulations of pharmaceuticals was introduced in the late 1940 's and early 1950 's. Today, many solid dosage forms utilize pellets or spheres as the basis for the controlled release of drugs. Pellets have several properties which make them particularly useful as pharmaceuticals. As a consequence of their geometry, spheres have a minimum surface area per unit of volume, and their well-characterized, regular shape facilitates mass-transfer modeling. Spheres make excellent candidates as drug carriers. Multiple-bead dosage forms may provide smoother absorption profiles (Robinson and Hollenbeck, 1991) and their superior flow characteristics make them nearly ideal for processing.

One of the most useful and well-known spheronizing agents in use is microcrystalline cellulose (Avicel Spheres, FMC Corp.). It is a non-fibrous form of cellulose in which the cell wall of plant fibers is broken into fragments by hydrolysis with dilute mineral acid (Swarbrick and Boylan 1988, p.46). This aqueous slurry is purified and spray-dried, forming the microcrystalline powder. By controlling the atomization and drying, the particle size distribution can be varied. Chemically, it is identical to natural cellulose; only the physical form is changed from fibers to particles. Chemical analysis reveals a very high molecular-weight polysaccharide composed exclusively of glucose units. Hydrogen bonds between hydroxyl groups on adjacent cellulose molecules are thought to be responsible for the strength of microcrystalline cellulose (ibid).

Several types of microcrystalline cellulose (MCC) are available from different suppliers. One popular brand Avicel® (FMC Corp.) is available in several different grades. Powdered grades, such as Avicel-PH grades 101, 102, 103, 105 and 200, differ primarily in particle size. Among these, Avicel $\mathrm{PH}-101$ is the one often chosen for direct 
tableting and wet granulation processing, but its relatively small particle size (typically 50 microns) makes its flow properties inferior to larger-sized grades, such as Avicel PH-102. The recent introduction of Avicel PH-200 was designed to offer still larger-sized particles and yet better flow than Avicel PH-102. Newton, Chow and Jeewa (1993) recently investigated various powdered and colloidal grades (containing $\mathrm{MCC}$ and $\mathrm{NaCMC}$ ) of microcrystalline cellulose from different suppliers. Significant differences were found between grades and between suppliers for both powdered and colloidal grades when used in an extrusion-spheronization process, even between grades promoted as 'interchangeable'. Other investigators have tried novel materials as binding-spheronizing agents such as chitosan (Goskonda and Upadrashta, 1993), or have attempted higher levels of drug loading (Hileman, et al. (1993). Vecchio, et al. (1994) also attempted high levels of drug loading with indobufen, but found that a $30 \% \mathrm{MCC}$ content was required for high quality spheres.

Previous investigations have focused primarily on pelletization by the traditional four-step method of granulation, extrusion, spheronization and drying. Woodruff and Nuessle (1972) investigated rotational speed and dwell time during marumerization. Malinowski and Smith (1975) applied a factorial experimental approach to evaluate five process variables in a marumerization process. More recently, Hileman, et al. (1993) used a Plackett-Burman design to investigate multiple variables for a similar process using a $\mathrm{Nica}^{\mathrm{TM}}$ spheronizer. Other investigators have also studied variables such as the amount and composition of granulating fluid (Elbers, Bakkenes and Fokkens 1992, Otsuka, Gao and Matsuda 1994) and spheronization and drying conditions (Bataille, et al., 1993). Fewer studies have been published concerning newer processors which use fluidized air columns outfitted with rotary inserts for one-step granulation-spheronization and drying. The Niro Aeromatic $\circledast$ and the Glatt $\circledast$ Rotoprocessor combine the four traditional steps into one convenient unit operation. Vecchio, et al. (1994) used the Niro ${ }^{\mathrm{TM}}$ processor to prepare 
indobufen pellets without starting seeds. Wan, Heng and Liew (1994) studied the role of moisture and gap air flow in the same equipment. Robinson and Hollenbeck (1991) compared extrusion-spheronization techniques with fluid-bed rotor processing using the Aeromatic, finding that the methods produced equivalent results.

Very little has been published on the use of the Glatt Rotoprocessor ${ }^{\mathrm{TM}}$. Jäger and Bauer (1982) published the first report. Subsequently, O'Connor, Remon and Schwartz compared 10\% drug-loaded spheres using several techniques (Avicel@ Spheres, FMC Corp.), including marumerization and rotoprocessing. The present work will study the new spheronizing agent Avicel $200 \circledR$ in a rotary fluid-bed pelletization process. High (e.g. 90\%) drug loading will be attempted using substantially different physical grades of both MCC and model drug. No studies of the pelletization process to date have focused on the physical grades of drug substance as well as spheronizing agent. Differences in size, shape and flow might have significant effects on the agglomeration process for binary systems, particularly when drug loading levels reach extreme levels.

The main purposes of this study are to;

1) Identify differences between three grades of Avicel $@$ and to test their relative suitability for a spheronization.process

2) Identify differences between three grades of anhydrous theophylline and to test their relative suitability for a spheronization.process

3) Compare pellets made with three grades of anhydrous theophylline and three grades of Avicel at the drug loading levels of $50 \%$

4) Study the effects of varying degrees of drug loading, from $25 \%$ to $90 \%$, on the quality of pellets made with a single grade of anhydrous theophylline 
EXPERIMENTAL

\section{Experimental Design}

To test the physical and chemical properties of spheres made with all possible combinations of grades of anhydrous theophylline with microcrystalline cellulose at a $1: 1$ ratio, the following two-factor, three level full factorial design in one replicate was used:

\begin{tabular}{|c|c|c|c|}
\hline & \multicolumn{3}{|c|}{ THEOPHYLLINE GRADE } \\
\hline AVICEL GRADE & Type A & Type B & Type C \\
\hline Avicel PH-101 & & & \\
\hline Avicel PH-102 & & & \\
\hline Avicel PH-200 & & & \\
\hline
\end{tabular}

The response variables measured were:

- Size uniformity

- Shape factor

- Shape uniformity

- Flowability

- Friability

- Theophylline Content

A second experiment focusing on the effect of drug loading upon pellet shape was performed. The design for this experiment is found in the section "Effect of Drug Loading on Sphere Shape". 


\section{Materials}

Anhydrous theophylline was available in three particle size grades (Knoll AG, Germany). 'K-205' was large-sized (Type 'A'), 'Micro' was micronized (Type 'C'), and 'R-404' (Type 'B') was intermediate. Avicel® PH-101, PH-102 and PH-200 were available from FMC Corporation, Philadelphia, PA. All spheronization was performed with Purified Water as the granulating agent.

\section{Methods}

Raw Material Evaluation

Anhydrous theophylline and Avicel were analyzed for particle size and shape using scanning electron microscopy (JEOL, USA) in conjunction with quantitative image analysis (SPI Supplies, West Chester, PA) available at Structure Probe (Metuchen, NJ).

\section{Manufacturing Process}

Spheronization was performed using a Glatt GPCG-1 Versaglatt (Glatt Air Techniques, Ramsey, NJ) fitted with a 12 -inch rotor insert with waffle plate. The interior of the fluid-bed column contained one tangentially-mounted Schlick® nozzle operating at 1.5 bar pressure. Water was sprayed at a rate of $50-60 \mathrm{ml} / \mathrm{min}$ using a Masterflex Pump and Flow Controller.

\section{Bulk Density}

Loose and tapped bulk density was determined using a Vanderkamp Tap Density Tester Model 10700 (VanKel Industries, Chatham, NJ). 
True Density

True density determinations were made using a Micromeritics Autopycnometer Model 1320 (Micromeritics Corp., Norcross, GA) using helium as the intrusive gas.

\section{Sphere Flowability}

Flow rate of pellets was determined using an Erweka Flow Tester Model GWF (Erweka Instruments, Milford, CT). The flow rate of one hopper-sized sample is measured and the reading converted to a flowability value by calculating the reciprocal angle of repose.

\section{Friability}

Six grams of pellets larger than 30 -mesh are dedusted by sieving for two minutes using an ATM Sonic Sifter. Six grams of $3 \mathrm{~mm}$ glass beads (Kimble $($ ) are added to the sample and rotated for ten minutes in an 8-inch baffled abrasion drum operating at $25 \mathrm{rpm}$. The pellets are again dedusted and reweighed; the weight loss is expressed as a percentage loss.

\section{Shape Factor (Sphericity)}

A random sample of thirty untreated pellets of mesh size smaller than 18 but larger than 30-mesh (US Standard sieves) are mounted on a microscope slide. Images taken through a Nikon Microphot FXA Microscope (Nikon Corp., Tokyo, JAPAN) are analyzed using the Microcomp Integrated Image Analysis System (Southern Micro Instruments, Inc., Atlanta, GA). 
SEM

Scanning electron micrographs were produced using the Jeol JSM-6100 Microscope (Jeol, Tokyo, JAPAN).

\section{Drug Content}

Theophylline potency in pellets is quantitatively determined by high-performance liquid chromatography (Perkin Elmer) in conjunction with a UV detector measuring absorbance set at $275 \mathrm{~nm}$ for potency assay and $220 \mathrm{~nm}$ for purity determination. 


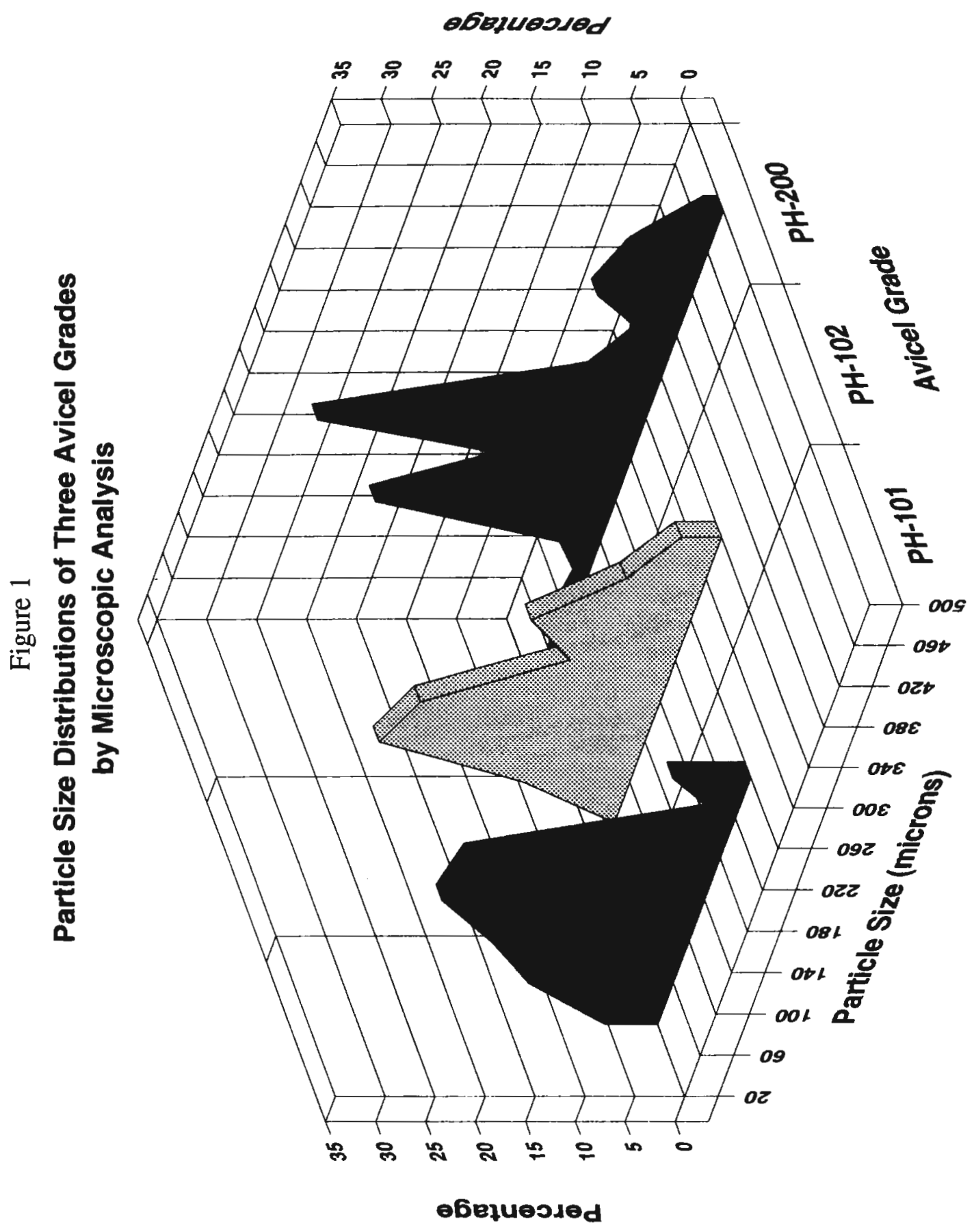


Figure 2

Shape Factor (Sphericity) Distribution for Three Avicel Grades

$($ circle $=1$, line $=0)$

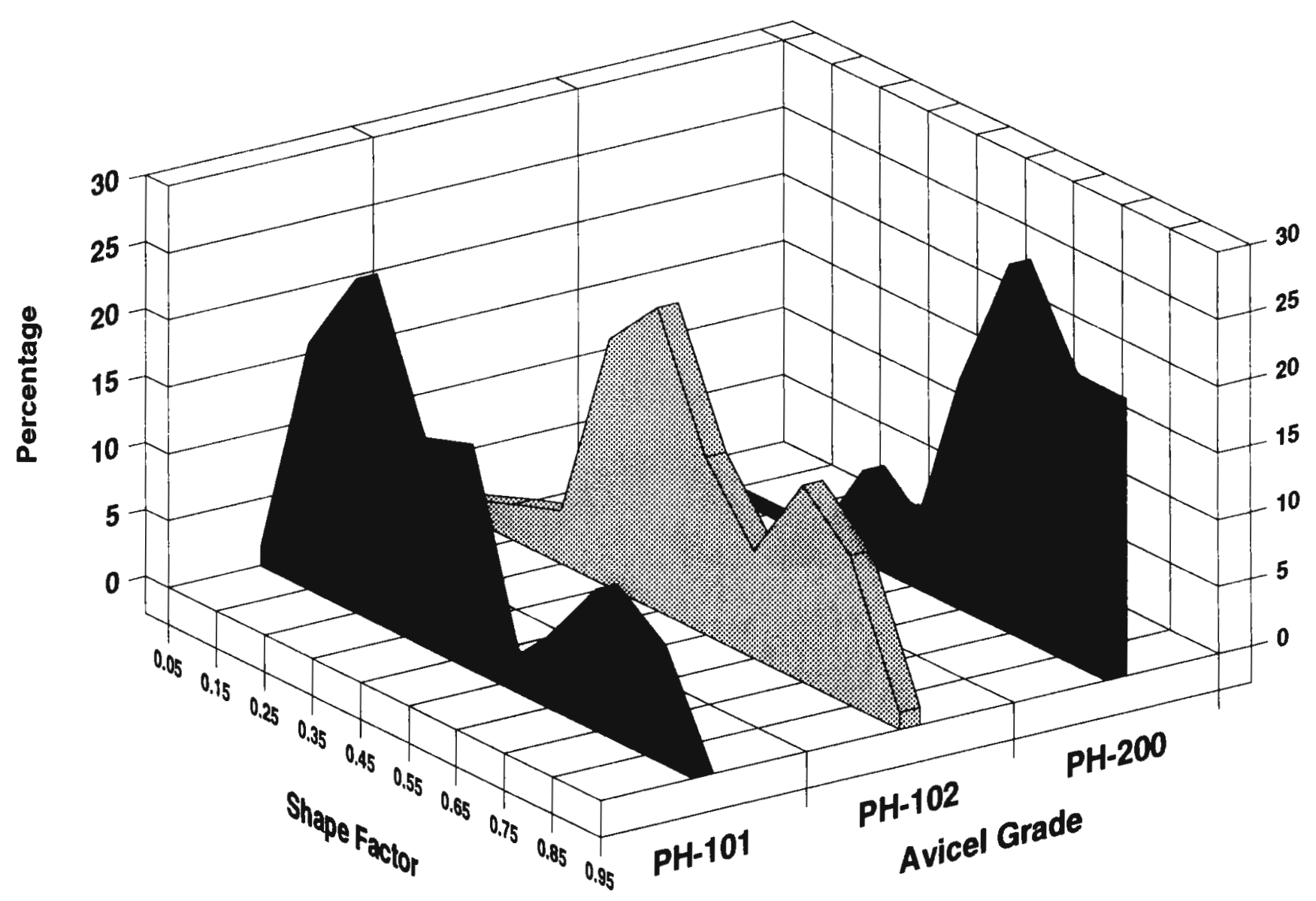


Figure 3

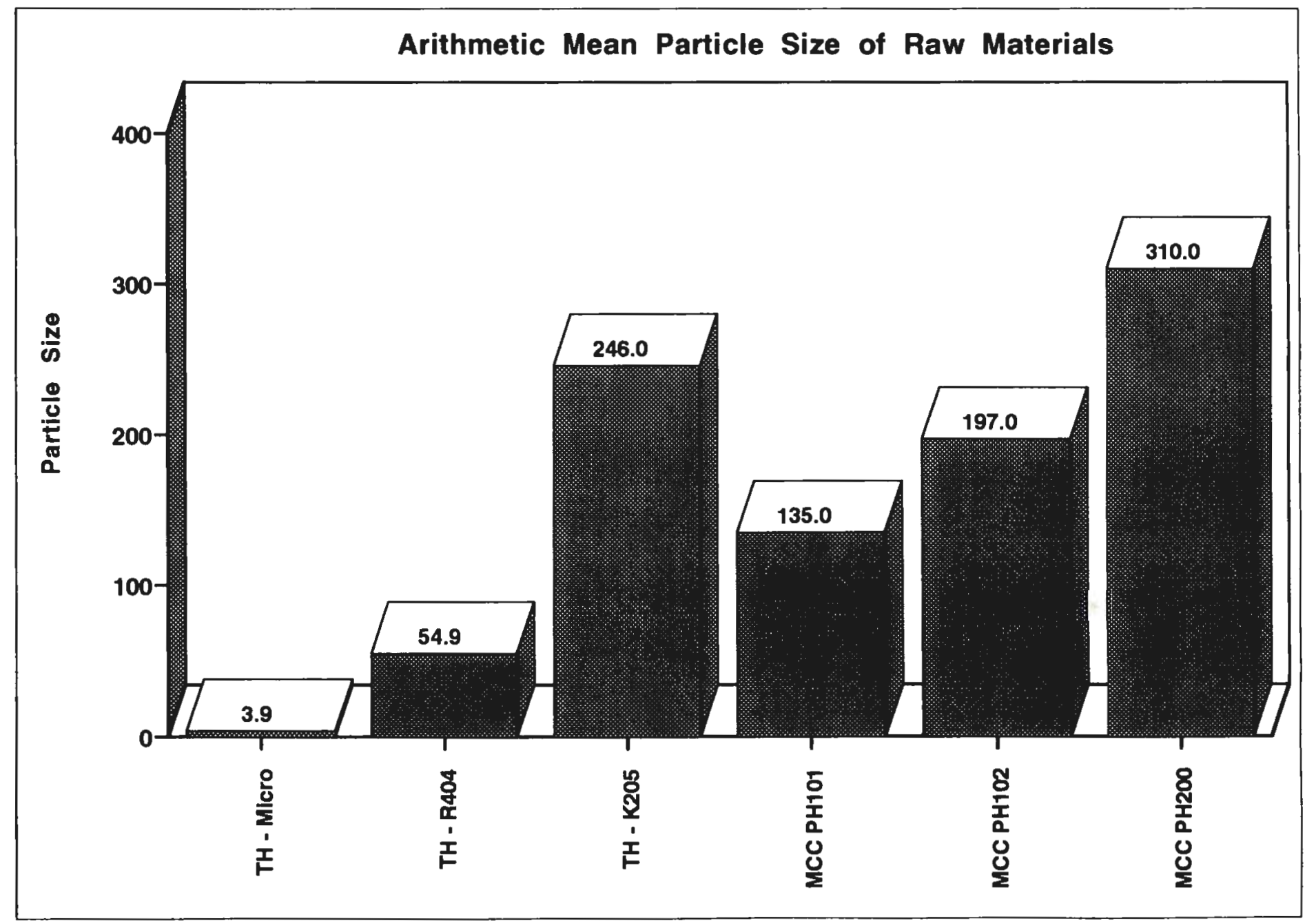




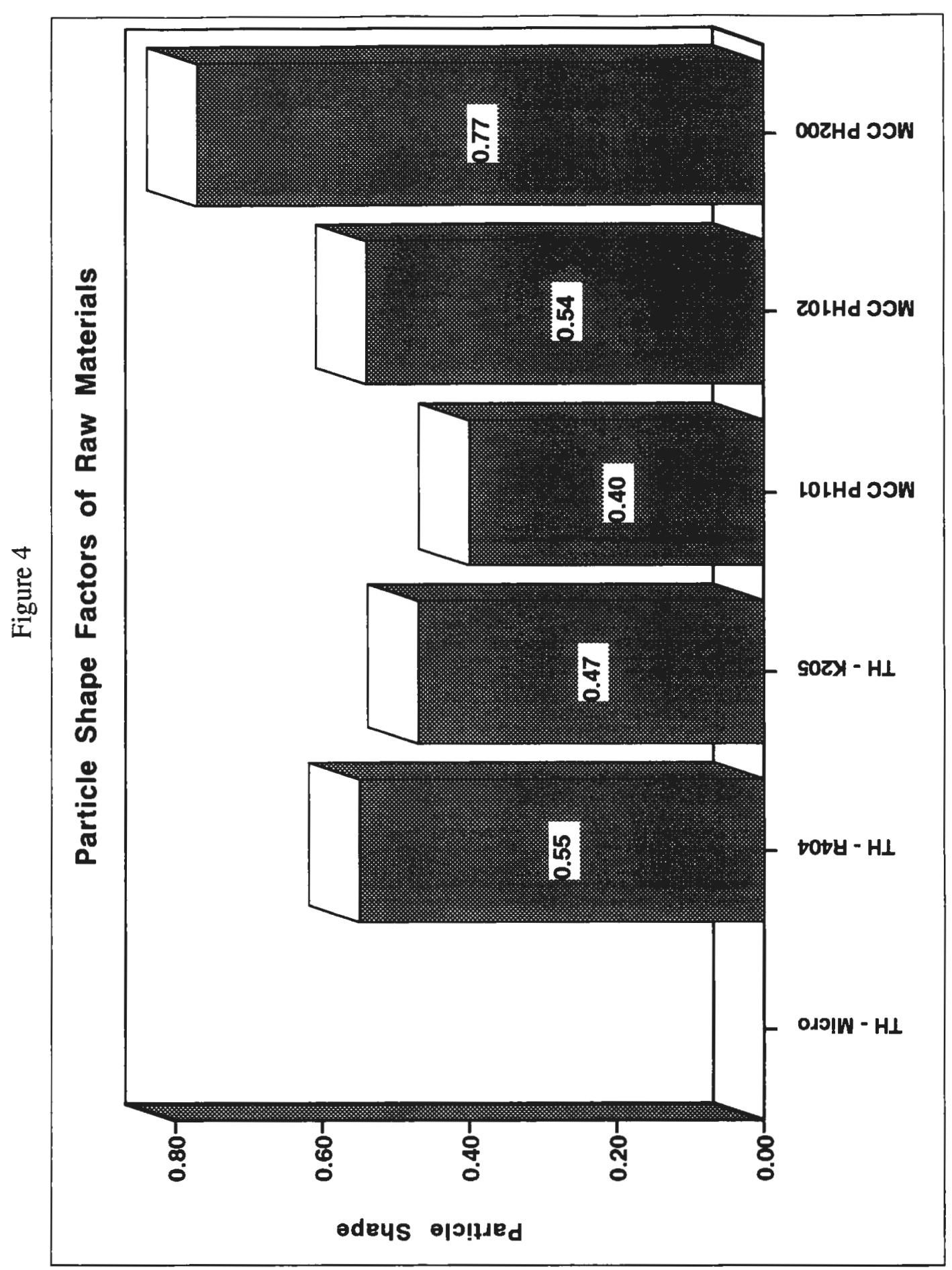


RESULTS AND DISCUSSION

Sphere Size Uniformity

It is sometimes desirable to produce pellets that are essentially equal in size. A particular size pellet may be chosen as optimal for a certain duration of drug release, for example. Sizes above and below the range would then either shorten or extend release of the drug. In this experiment the goal was to optimize batch yields in the $18 / 20$ to $35 / 40$ size range (US Standard Sieve Sizes, PASS/RETAIN), and to create as narrow a size distribution as possible.

The particle sizes of individual spheres were measured using optical microscopic methods. The geometric mean size is calculated from the number-size distribution. The size uniformity of the spheres was evaluated by calculating the standard deviation of the geometric mean particle size. The formulas for both geometric mean size and standard deviation are given in Manuscript I, Experimental Section.

The results indicate no significant differences between the mean size uniformities of spheres made with different Avicel and theophylline grades. Each mean represents the average of three batches. Scheffe's tests show no differences (means with the same letter are not different), and a regression on the two variables is not significant $(p=0.10)$. Results are summarized in Table 1 below. 
Table 1

Size Uniformity (Geometric Mean Size Standard Deviation) of 50\% Avicel-Theophylline Spheres: The Effect of Raw Material Grade

\begin{tabular}{|l|c|c|c|}
\hline AVICEL GRADE & Mean & Std. Dev. & $\begin{array}{c}\text { Scheffe } \\
\text { Grouping }\end{array}$ \\
\hline Avicel PH-101 & 1.37 & 0.07 & $\mathrm{~A}$ \\
\hline Avicel PH-102 & 1.21 & 0.05 & $\mathrm{~A}$ \\
\hline Avicel PH-200 & 1.30 & 0.07 & $\mathrm{~A}$ \\
\hline THEOPHYLLINE TYPE & & & \\
\hline Type A & 1.26 & 0.114 & $\mathrm{~A}$ \\
\hline Type B & 1.27 & 0.024 & $\mathrm{~A}$ \\
\hline Type C & 1.34 & 0.113 & $\mathrm{~A}$ \\
\hline
\end{tabular}

(means with the same letter are not significantly different) 
Sphere Shape and Shape Uniformity at 50\% Drug Loading

Quantitative image analysis was performed on scanning electron microscopic images of individual spheres. Each sphere was characterized by a 'shape factor' equivalent to its sphericity. Target values range from zero to one $(0-1)$, one being the theoretical maximum value.

Sample size was approximately 100 spheres per batch tested. For each batch, a mean sphericity and its standard deviation is calculated and presented in Table 2. This standard deviation is the uniformity of the shape factors and is reported as the Uniformity. Shape uniformity is reported as the mean of three batches. The standard deviation of the uniformity is also calculated (the 'standard deviation of the standard deviations') and is listed in the next to last column. This allows a Scheffe's Test to compare the uniformity among all batches.

Results indicate that all nine combinations of Avicels and theophylline grades made spheres whose shape characteristics were not statistically differentiable from each other. 


\section{Table 2}

Sphere Shape and Shape Uniformity of 50\% Avicel-Theophylline Spheres:

The Effect of Raw Material Grade

\begin{tabular}{|l|c|c|c|c|c|}
\hline AVICEL GRADE & $\begin{array}{c}\text { Mean } \\
\text { Sphericity }\end{array}$ & $\begin{array}{c}\text { Scheffe } \\
\text { Grouping }\end{array}$ & Uniformity & Std. Dev. & $\begin{array}{c}\text { Scheffe } \\
\text { Grouping }\end{array}$ \\
\hline Avicel PH-101 & 0.867 & $\mathrm{~A}$ & 1.101 & 0.035 & $\mathrm{~A}$ \\
\hline Avicel PH-102 & 0.861 & $\mathrm{~A}$ & 1.104 & 0.008 & $\mathrm{~A}$ \\
\hline Avicel PH-200 & 0.861 & $\mathrm{~A}$ & 1.113 & 0.042 & $\mathrm{~A}$ \\
\hline $\begin{array}{l}\text { THEOPHYLLINE } \\
\text { GRADE }\end{array}$ & & & & & \\
\hline Type A & 0.857 & $\mathrm{~A}$ & 1.119 & 0.030 & $\mathrm{~A}$ \\
\hline Type B & 0.874 & $\mathrm{~A}$ & 1.078 & 0.015 & $\mathrm{~A}$ \\
\hline Type C & 0.858 & $\mathrm{~A}$ & 1.122 & 0.015 & $\mathrm{~A}$ \\
\hline
\end{tabular}

(means with the same letter are not significantly different) 
Compressibility Index

The compressibility index indicates the potential for granulates to flow (Carr, 1965). Higher values indicate a decreased potential for flowability. All spheres demonstrated very low values for compressibility, suggesting a high potential for flow. This is expected for such highly idealized materials such as hard, relatively spherical pellets in the $0.5-1.0 \mathrm{~mm}$ size range.

Pellets made with Avicel PH-101 appear to be slightly more compressible than those made with the larger grades, as indicated by the different Scheffe groupings in Table 3. These higher compressibility values may result from differences in pellet size uniformity. Avicel PH-101 had the largest size dispersity (1.37); Avicel PH-102 had the lowest (1.21). Avicel PH-200 had intermediate values of both. Although the size dispersities themselves were not significantly different, they may nevertheless help explain the differences in compressibility. Pellets with a range of sizes rearrange to pack more closely as small particles to fill the void space between larger particles.

Despite these differences in compressibility values, actual flowability measurements using the funnel flow technique reveal no differences between Avicels or theophylline grades. 


\section{Table 3}

Compressibility Index (CI, \%) for 50\% Avicel-Theophylline Spheres:

The Effect of Raw Material Grade

\begin{tabular}{|l|c|c|c|}
\hline AVICEL GRADE & CI (\%) & Std. Dev. & $\begin{array}{c}\text { Scheffe } \\
\text { Grouping }\end{array}$ \\
\hline Avicel PH-101 & 7.28 & 1.48 & $\mathrm{~A}$ \\
\hline Avicel PH-102 & 4.24 & 0.95 & $\mathrm{~B}$ \\
\hline Avicel PH-200 & 5.33 & 1.14 & $\mathrm{~B}$ \\
\hline THEOPHYLLINE TYPE & \multicolumn{3}{|}{} \\
\hline Type A & 5.07 & 1.72 & $\mathrm{~A}$ \\
\hline Type B & 6.06 & 1.99 & $\mathrm{~A}$ \\
\hline Type C & 5.71 & 1.50 & $\mathrm{~A}$ \\
\hline
\end{tabular}

(means with the same letter are not significantly different) 
Sphere Friability

Inasmuch as drug-containing spheres are generally intended for further processing, such as coating, the strength and integrity of the sphere and its surface are important. Uniform spherical particles with smooth, durable surfaces facilitate the uniform application of polymeric materials.

There is no uniformly accepted method for testing pellet friability. One technique, however, is to subject samples of pellets to abrasion by impact with larger beads of glass or metal in a rotating drum (Jäger and Bauer, 1982).

Pellets from each of the nine combinations of Avicel and theophylline are tested in triplicate. Pellets containing no drug (Theophylline type 'D') are used as a control, since presumably, excessive drug loading may weaken pellet strength.

A regression analysis on the two main factors AVICEL TYPE and THEOPHYLLINE TYPE show both to be only very weakly significant ( $p=0.049$ and $p=0.048$, respectively). Table 4 results of Scheffe's Test performed on both factors show no differences between Avicels. Slight differences between theophylline grades follow no discernable pattern with regard to particle size, the presence or absence of drug loading, or even the amount of water used in the manufacturing process. The apparent differences in friability may more likely be statistical artifact, or due to some other factor. 
Table 4

Sphere Friability (\%) for 50\% Avicel-Theophylline Spheres:

The Effect of Raw Material Grade

\begin{tabular}{|l|c|c|c|}
\hline AVICEL GRADE & Mean & Std. Dev. & $\begin{array}{c}\text { Scheffe } \\
\text { Grouping }\end{array}$ \\
\hline Avicel PH-101 & 2.10 & 1.70 & A \\
\hline Avicel PH-102 & 1.93 & 1.29 & A \\
\hline Avicel PH-200 & 2.99 & 1.31 & A \\
\hline THEOPHYLLINE TYPE & \multicolumn{3}{|l}{} \\
\hline Type A & 3.07 & 2.19 & A \\
\hline Type B & 1.40 & 1.12 & B \\
\hline Type C & 2.48 & 1.13 & A, B \\
\hline Type D (0\% theophylline) & 2.19 & 0.84 & A, B \\
\hline
\end{tabular}

(means with the same letter are not significantly different) 
Sphere Theophylline Content

The potency of the theophylline-loaded pellets, as a percentage of the claimed drug content, was measured by HPLC assay methodology. Pellet batches made with the three grades of theophylline and MCC were formulated to contain drug loading levels from $20 \%$ to $90 \%$. Each batch was fractionated by sieve size into three groupings A, B and C. The results were then analyzed by a four-way classification analysis of variance to determine four main effects AVICEL TYPE, THEOPHYLLINE TYPE, SIEVE CUT and DRUG LOADING LEVEL.

ANOVA results show drug loading levels to be the most significant factor affecting the capacity of pellets to retain drug during processing $(p<0.0001)$. Actual potency decreases as theoretical potency increases. This is consistent with the observed tendency of theophylline to adhere to interior surfaces of the processor, moreso than Avicel, causing a relative increase in the proportion of cellulose remaining in the pellet.

It might be expected that the more adhesive micronized theophylline (Type C) would show a greater propensity for loss during pelletization, but this does not appear to be the case. Results in Table 5 show mean assay values for theophylline Types A, B and C to be $91.4 \%, 92.0 \%$ and $93.0 \%$, respectively. Actually, however, it was not possible to manufacture pellets with theoretical potencies exceeding $50 \%$ with Type $\mathrm{C}$ due to severe adhesion to the walls and rotor plate; likewise, it was not possible to exceed a $70 \%$ potency using Type B for the same reason. The data for the most potent pellets, therefore, is derived only from pellets made with theophylline types $\mathrm{A}$ (and some type B). It was also not possible, therefore, to evaluate the effects of Avicel type on potency decreases with increasing drug loading levels (an interaction effect between the factors AVICEL TYPE and theoretical POTENCY) because of the difficulties in manufacturing candidate batches. 
The results shown in Table 5 indicate a significantly lower potency for batches made with Avicel PH-200. This would seem an unexpected result, since this grade of Avicel was the most amenable to processing (as evidenced by its ability to pelletize even at the highest drug loading levels). Batches manufactured using this grade of Avicel showed the least tendency to adhere to surfaces, thereby helping to maintain the intended potency levels. Actually, however, assay values which significantly exceed $90 \%$ of theoretical, for example, may indicate a tendency to lose Avicel, not an increased ability to retain theophylline. Similarly, assay values for Avicel PH-200 in the $89 \%$ range indicate that little or no Avicel is lost, while a small amount of theophylline is lost by adhesion to the column interior during fluidization.

There is no evidence that the drug content varies with the sieve cut of a given batch. Assay values in Table 6 show the mean assay values to be similar. Table 6 also shows that pellets of all strengths retain their drug content about equally well, with mean theophylline content not falling below $89.38 \%$ theoretical for any one potency. 
Table 5

Pellet Theophylline Content (\% Theoretical) for Avicel-Theophylline Spheres:

The Effect of Raw Material Grade

\begin{tabular}{|l|c|c|c|}
\hline AVICEL GRADE & Mean & Std. Dev. & $\begin{array}{c}\text { Scheffe } \\
\text { Grouping }\end{array}$ \\
\hline Avicel PH-101 & 92.2 & 3.11 & A \\
\hline Avicel PH-102 & 92.0 & 0.93 & A \\
\hline Avicel PH-200 & 89.1 & 3.84 & B \\
\hline THEOPHYLLINE GRADE & \multicolumn{3}{|l}{} \\
\hline Type A & 91.4 & 2.63 & A \\
\hline Type B & 92.0 & 2.77 & A, B \\
\hline Type C & 93.0 & 4.15 & B \\
\hline
\end{tabular}

(means with the same letter are not significantly different) 
Table 6

Pellet Theophylline Content (\% Theoretical) for Avicel-Theophylline Spheres:

The Effect of Sphere Size Fraction (Sieve Cut) and Percent Drug Loading

\begin{tabular}{|c|c|c|c|}
\hline SIEVE CUT & Mean & Std. Dev. & $\begin{array}{c}\text { Scheffe } \\
\text { Grouping }\end{array}$ \\
\hline $\mathrm{A}$ & 92.54 & 2.22 & $\mathrm{~A}$ \\
\hline $\mathrm{B}$ & 92.90 & 3.02 & $\mathrm{~A}$ \\
\hline $\mathrm{C}$ & 92.90 & 3.33 & $\mathrm{~A}$ \\
\hline POTENCY & \multicolumn{3}{|l}{} \\
\hline $20 \%$ & 90.47 & 2.52 & $\mathbf{B}, \mathrm{C}$ \\
\hline $25 \%$ & 95.8 & 1.83 & $\mathrm{~A}$ \\
\hline $40 \%$ & 91.89 & 4.01 & $\mathbf{B}$ \\
\hline $50 \%$ & 91.29 & 2.16 & $\mathbf{B}, \mathrm{C}$ \\
\hline $70 \%$ & 90.36 & 1.61 & $\mathbf{B}, \mathbf{C}$ \\
\hline $90 \%$ & 89.38 & 0.62 & $\mathrm{C}$ \\
\hline
\end{tabular}

(means with the same letter are not significantly different) 


\section{Pellet Shape}

A separate experiment was performed to investigate in more detail the effect of drug potency on sphericity. To the unaided eye, pellets containing 50\% drug loading or less appeared round and elegant, while those containing $70 \%$ or $90 \%$ were often judged to be more granular than spherical. In this experiment, five levels of drug loading were used in conjuction with the three Avicel grades; all batches used only Type A $(K-205)$ theophylline. Type A was chosen because it was not possible to manufacture pellets of all strengths using any other grade of theophylline. The experimental design is shown below in Figure 5. Each combination was manufactured in triplicate (labeled A, B and C) for a total of 45 independent experimental trials.

Pellets were examined using a light microscope fitted with a video camera. Images were transferred to a desktop computer where the image was digitized. Image analysis software then calculated a sphericity shape factor using the following formula:

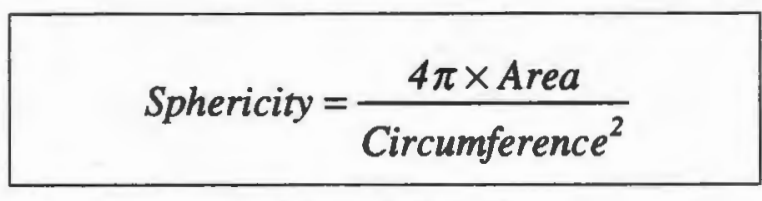

(circle $=1$, line $=0$ )

Each batch was evaluated by examining 30 pellets individually; for each batch a mean and standard deviation was calculated. The data was analyzed using a two-way classification ANOVA using the following model:

Sphericity $=f($ Avicel Grade, Drug Concentration $)$ 
The results in Table 7 show that pellet shapes are identical for the three Avicel grades, while high levels of drug loading (e.g. $70 \%$ and $90 \%$ ) result in a significant deterioration of pellet sphericity. A notable exception is $0 \%$ drug loading, which also has lower values not different from the $70 \%$ pellets. The reason for this is not readily apparent to the naked eye. Pure cellulosic spheres appeared very round indeed; on SEM examination, however, it could be seen that small pieces of cellulosic dust had adhered to their surfaces. Digitized images of these apparently very round pellets could not differentiate the sphere surface itself from the adhering particles. Measurements of the pellet circumferences therefore included perimeters of individual contaminant particles, resulting in artificially large values for each sphere. This, in turn, resulted in falsely low shape factors for each. 
Figure 5

EXPERIMENTAL DESIGN

Sphere Shape for Three Avicel Grades versus Percentage Drug Loading (Type 'A' (K-205) Theophylline Only)

\begin{tabular}{|c|c|c|c|c|c|}
\hline & \multicolumn{5}{|c|}{ THEOPHYLLINE POTENCY } \\
\hline & $0 \%$ & $25 \%$ & $50 \%$ & $70 \%$ & $90 \%$ \\
\hline \multirow{3}{*}{ Avicel PH-101 } & $A$ & $A$ & $A$ & $A$ & $A$ \\
\hline & $B$ & $B$ & $B$ & $B$ & $B$ \\
\hline & C & C & $C$ & C & $C$ \\
\hline \multirow{3}{*}{ Avicel PH-102 } & $A$ & $A$ & $A$ & $A$ & $A$ \\
\hline & $B$ & $B$ & $B$ & $B$ & $B$ \\
\hline & $C$ & C & C & C & C \\
\hline \multirow{3}{*}{ Avicel PH-200 } & $A$ & $A$ & $A$ & $A$ & $A$ \\
\hline & $B$ & $B$ & $B$ & $B$ & $B$ \\
\hline & $C$ & $C$ & $C$ & $C$ & $C$ \\
\hline
\end{tabular}




\section{Table 7}

Pellet Shape Factors (Sphericity) for Avicel-Theophylline* Spheres:

The Effect of Avicel $®$ Grade and Percent Drug Loading

\begin{tabular}{|c|c|c|c|}
\hline AVICEL GRADE & Mean & Std. Dev. & $\begin{array}{c}\text { Scheffe } \\
\text { Grouping }\end{array}$ \\
\hline Avicel PH-101 & 0.824 & 0.035 & $\mathrm{~A}$ \\
\hline Avicel PH-102 & 0.824 & 0.043 & $\mathrm{~A}$ \\
\hline Avicel PH-200 & 0.824 & 0.032 & $\mathrm{~A}$ \\
\hline POTENCY & \multicolumn{3}{|}{} \\
\hline $0 \%$ & 0.830 & 0.038 & $\mathrm{~A}$ \\
\hline $25 \%$ & 0.836 & 0.018 & $\mathrm{~A}$ \\
\hline $50 \%$ & 0.844 & 0.020 & $\mathrm{~A}$ \\
\hline $70 \%$ & 0.828 & 0.027 & $\mathbf{A , B}$ \\
\hline $90 \%$ & 0.785 & 0.044 & $\mathbf{B}$ \\
\hline
\end{tabular}

(* theophylline type 'A' (K-205) only)

(means with the same letter are not significantly different) 
DISCUSSION

The spheronization of theophylline with microcrystalline cellulose was carried out using a recently introduced Glatt ${ }^{\mathrm{TM}}$ fluid-bed Rotogranulator. Three grades of anhydrous theophylline and three grades of microcrystalline cellulose, differing considerably in size and shape (but not chemically) are used in all possible permutations and in varying proportions, from $0 \%$ drug loading to $90 \%$. The pellets are evaluated for their size, shape (sphericity), density, uniformity of size and shape, friability, flowability, and drug content.

The most notable finding was that for many of the combinations of materials it was not possible to produce spherical pellets at all. This immediately limited the possibilities for completing an experiment which tested pellet quality for all materials at all drug loading levels. In the worst case, the micronized anhydrous theophylline Type ' $\mathrm{C}$ ' (the micronized grade), when combined with Avicel PH-101, spheronized well at $25 \%$ drug loading, but very poorly at 50\%. In the best case, that of Avicel PH-102 and theophylline Type ' $\mathrm{A}$ ' (the coarsest grade, also refered to as $\mathrm{K}-205$ ), it was possible to make acceptable spheres without difficulty (such as interrupting the process to remove adhesions) at $90 \%$ loading. The experimental strategy, therefore, was first to examine all combinations of materials (nine in all) at nominal levels of drug loading (e.g. $50 \% \mathrm{~W} / \mathrm{W}$ ) to evaluate the effects of using three different physical grades of Avicel@-brand microcrystalline cellulose (MCC) with three physically distinct grades of anhydrous theophylline.

A second experiment was designed to test the effects of drug loading levels on pellet properties such as potency retention and shape. Since it was only possible to produce pellets of $90 \%$ drug using one grade of theophylline (Type 'A'), the experiment was designed (Figure 5) to include Type A theophylline with all three Avicels at levels of $0 \%$ (control), $25 \%, 50 \%, 70 \%$ and $90 \%$. 
The potential of these materials to spheronize is inversely related to their particle size. Most importantly, it was the grade of theophylline which controlled the ease of processing. Theophylline Type $\mathrm{C}$ had an arithmetic mean particle size of less than 4 microns (Figure 3). The intermediate grade of theophylline (Type B) had a mean size of 54.9 microns, while the most favorable grade Type A had a mean size of 246 microns. Of the four possible drug loading levels ( $25 \%-90 \%)$, Type C pelletized at only two of them (a 70\% pellet was made using Avicel PH-200, but only with great difficulty). By contrast, Type B theophylline, the intermediate grade, spheronized at three of the four levels (up to 70\%) with all three Avicel grades. Finally, Type A spheronized with all three Avicels at all four strengths.

All three Avicel grades spheronized well. The three grades differed in particle size only slightly (by comparison with the three theophyllines). Avicel PH-101 had an arithmetic mean size of 135 microns, compared with Avicel PH-200, which had a mean size of 310 microns (see Figure 3). Nevertheless, there were measurable differences between them. Of the twelve possible combinations with each Avicel (three theophyllines and four drug loading levels), it was possible to spheronize nine of them (75\%) using Avicel PH-101 and Avicel PH-102. Avicel PH-200 was able to form pellets in ten of the twelve cases (83\%). A subjective evaluation of the ease of processing, as measured by the number of times the process required interruption for scraping material from the column walls, indicated that PH-102 may be slightly more amenable to processing than PH-200.

The results of the first investigation using all nine combinations of materials (three Avicels and three theophyllines, all at 50\% drug loading levels) reveal that there were relatively few differences between them. The pellet size uniformity (the narrowness of the particle size distribution) was similar for all grades of materials tested. There were no differences in their shape factors (e.g. roundness), or shape uniformity. The 
compressibility index, which indicates a potential for flowability, was slightly higher (worse) for Avicel PH-101, but was not related to theophylline grade. Actual flow measurements (not shown) using funnel flow techniques failed to show any differences between Avicels or theophyllines.

A high resistance to abrasion is a desirable attribute for pellets, since they generally undergo further processing and because their integrity is important for controlled drug delivery systems. There were no marked differences between grades.

In most instances, the ability of the pellets to retain drug during the agglomeration process did not differ between grades of material, between size fractions, or for different drug loading levels. All pellets tested retained approximately $90 \%$ of the drug, although larger grades of Avicel produced pellets of slightly lower drug content (about 1\%). This may be related to a loss of lighter (smaller) Avicel particles from the bed during fluidization, leaving behind a slightly increased proportion of drug. Avicel PH-200 has many larger cellulose agglomerates which do not adhere to interior surfaces of the column as easily as the longer, smaller fibers found in Avicel PH-101.

Shape factor analysis of spheres of with drug concentrations of $20 \%$ to $90 \%$ showed a statistically significant decrease in roundness when concentrations exceeded 70\%. Although some pellets made with $90 \%$ drug were judged acceptable for some purposes, they were much less spherical on visual examination and by image analysis techniques. While mean shape factors for other potencies ranged from 0.828 to 0.844 , those containing $90 \%$ drug averaged only 0.785 , a marked decrease $(p=0.004)$. The worst average shape factor for a single batch was 0.695 (90\% drug, 10\% Avicel PH-102). By comparison, among the best pellets tested a shape factor of 0.868 was the highest attainable mean for any single batch, while some individual pellets had shape factors as high as 0.898 . 


\section{CONCLUSIONS}

1) The potential for spheronization of binary systems using anhydrous theophylline and microcrystalline cellulose depends primarily on the physical grade of theophylline.

2) Despite significant differences in particle size and shape, all three microcrystalline cellulose grades spheronized well. The two larger grades, Avicel PH-102 and PH200 , were judged slightly better overall, however, particularly when more difficult drug materials are used in the spheronization process.

3) At the $50 \%$ drug loading level, all nine combinations of the raw material grades produced acceptable pellets. Although some statistically significant differences were found, these were small and likely of little practical significance

4) Pellet sphericity is significantly affected by the amount of drug loading. Shape factors which indicate the roundness of individual pellets show a marked decrease when $70 \%$ drug loading levels are exceeded. 


\section{REFERENCES}

Avicel Spheres, FMC Corporation, Food and Pharmaceutical Products Division, Philadelphia, 1985.

Bataille B., Ligarski K., Jacob M., Thomas C., Duru C. (1993) Study of the Influence of Spheronization and Drying Conditions on the Physico-Mechanical Properties of Neutral Spheroids Containing Avicel PH-101 and Lactose, Drug Development and Industrial Pharmacy, 19(6), 653-671.

Carr R.L. (1965) Evaluating Flow Properties of Solids, Chemical Engineering, 72, 163168.

Elbers J.A.C., Bakkenes H.W., Fokkens J.G. (1992) Effect of Amount and Composition of Granulation Liquid on Mixing, Extrusion and Spheronization, Drug Development and Industrial Pharmacy, 18(5), 501-517.

Goskonda S.R., Upadrashta S.M. (1993) Avicel RC-591/Chitosan Beads by Extrusion/Spheronization Technology, Drug Development and Industrial Pharmacy, 19(8), 915-927.

Hileman G.A., Goskonda S.R., Spallito A.J., Upadrashta S.M. (1993) A Factorial Approach to High Dose Product Development by an Extrusion/Spheronization Process, Drug Development and Industrial Pharmacy, 19(4), 483-491. 
Jäger K.F., Bauer K.H. (1982) Effect of Material Motion on Agglomeration in the Rotary Fluidized-Bed Granulator, Drugs Made in Germany, 25, 61-65.

Malinowski H.J.,Smith W.E. (1975) Use of Factorial Design to Evaluate Granulations Prepared by Spheronization, Journal of Pharmaceutical Sciences, 64(10), 1688-1692.

Newton J.M.,Chow A.K., Jeewa K.B. (1993) The Effect of Excipient Source on Spherical Granules Made by Extrusion/Spheronization, Pharmaceutical Technology, 17(3) 166-174.

Otsuka M., Gao J., Matsuda Y. (1994) Effect of Amount of Added Water During Extrusion-Spheronization Process on Pharmaceutical Properties of Granules, Drug Development and Industrial Pharmacy, 20(19), 2977-2992.

Robinson R.L., Hollenbeck R.G. (1991) Manufacture of Spherical Acetaminophen Pellets:Comparison of Rotary Processing with Multiple-Step Extrusion and Spheronization, Pharmaceutical Technology, 15(5), 48-56.

Swarbrick J., Boylan J.C., ed (1988) Encyclopedia of Pharmaceutical Technology, Marcel Dekker, New York.

Vecchio C., Bruni G., Gazzaniga A. (1994) Preparation of Indobufen Pellets by Using Centrifugal Rotary Fluidized Bed Equipment Without Starting Seeds, Drug Development and Industrial Pharmacy, 20(12), 1943-1956. 
Wan L.S.C., Heng P.W.S., Liew C.V. (1994) The Role of Moisture and Gap Air Pressure in the Formation of Spherical Granules by Rotary Processing, Drug Development and Industrial Pharmacy, 20(16), 2551-2561.

Woodruff C.W., Nuessle N.O. (1972) Effect of Processing Variables on Particles Obtained by Extrusion-Spheronization, Journal of Pharmaceutical Sciences, 61(5),787790. 
APPENDIX I

DISCUSSION 


\section{DISCUSSION}

Pharmaceutical wet granulation is comprised of three essential components. Machine, material and process variables, alone or in combination, determine the physical and physicochemical properties which make the intermediate and final products suitable for pharmaceutical purposes. The number of factors and the complex nature of the relationships between them have made certain aspects of the wet granulation process more easily regarded as 'art' than science. Human judgement, in the absence of scientific data, is often used to determine certain critical aspects of the process, such as when the granulation 'end-point' has been reached. Although a satisfactory product may nevertheless often result, several key considerations can get ignored in the process. Firstly, although the product may meet whatever minimum standards have been established for it, there is often no effort made to manufacture the 'best' product possible. Moreover, there is usually little scientific data generated to predict what effect changes in any of the controllable variables may have on the product. Future efforts to scale-up the process or to transfer the technology become more difficult when the important factors have not been clearly understood and their effects quantified.

The results of the present work have served to identify important variables, quantify their effects on various output variables, predict outcomes for untested process conditions, and to differentiate both materials and equipment. In Manuscript I, for example, it was found that the amount of water used in the process outweighed all other factors. Mixing speed often had little, if any effect. It did, however, modify the effect of water addition on certain properties. Using data aquired in accordance with an efficient central-composite design, the effects of each variable on several output variables were mapped over the experimental region of factor space. Responses from several output variables were then 
combined, based upon the predictive regression equations, to find an optimum region and even a single optimum point. This strategy accomplished several goals. Firstly, it isolated combinations of factors which resulted in an optimum product. Secondly, the effects of moving away from the optimum combination on any of the output variables were estimated. For example, it can be seen from Table 5, Manuscript I that relaxing the constraints on the amount of water used $\pm 5 \%$ still resulted in an optimum product. On the other hand, constraints on the speed of mixing can be relaxed $\pm 15 \%$ without adversely affecting the product. This illustrates the relative insignificance of mixing speed as a factor.

The location of the optimum point also points out another important, although more subtle, result. The optimum combination of factors, namely $425 \mathrm{ml}$ of water and 60 RPM mixing speed, did not coincide with the suspected optimum conditions that led to the choice of the design center point. Information gained prior to data collection suggested that the center point (67 RPM, $485 \mathrm{ml}$ of water) represented a suitable 'end-point' for the process. Subsequently, however, experimental data revealed that an optimum product resulted from a substantially less granulated material. Thus, the 'best' product would likely not have been found, despite considerable prior experience with the product and with wet granulation in general, without a more systematic and scientific approach. This realization may indicate the need to more broadly define the term 'end-point' as the combination of input variables which results in an optimum product, not merely the one that 'look right' and appears, based upon training and experience, to give a 'good granulation'.

The kinetics of agglomeration, as described by Usteri and Leuenberger (1989), predict an exponential increase in particle size as a function of liquid saturation levels. This strong increase in particle size was duplicated fairly closely, as liquid was added, by the wet granulation process using the Double Planetary Mixer. This led to the conclusion that the mixer could not be considered high shear, since there was no evidence of particle size 
reduction. Results using the PowerMix and the TK Fiedler were dramatically different. Figures 3, Manuscript II and Figure 2, Manuscript III show particle size decreases with increases in mixing speed. Clearly, these processors can be differentiated and even classified on the basis of this shearing capability. The PowerMix, as it turned out, was considerably more effective than the Fielder. Moreover, the experiments revealed that, in general, the materials used could benefit substantially from undergoing such a process. All the formulations used in Manuscripts I, II and III showed improved characteristics following wet granulation, even though the Emcompress(B-Lactose combination in Manuscript I ordinarily suffices as a direct-compression blend.

Formal experimentation, followed by an analysis of significant effects, showed that some factors had little or no effect by themselves, but interacted strongly with other variables. These two-way, and sometimes three-way, interactions were used to differentiate between machine, material and process variables. For example, while Starch 1500 was shown to be superior overall in terms of compactability and flowability, Starch 1551 sometimes responded more strongly to changes in mixing speed and water levels. Figure 6, Manuscript III shows a substantially greater effect on disintegration time for Starch 1551 than for Starch 1500 with increases in mixing speed and water levels. This could indicate a superiority of Starch 1551 for processes that require higher levels of water addition than was used in the present experiments.

Interaction effects also clearly differentiated the three theophylline grades from each other in Manuscript IV. Whereas the effect of drug loading was to decrease the spheronization potential of Avicel-theophylline combinations generally, it was most pronounced for the micronized grade of theophylline, followed closely by the intermediate grade. The best grade (Type A, K-205) suffered comparatively little. Thus, there was an interaction between theophylline grade and the drug loading level, in that much depended 
upon exactly which combination of materials was chosen. At modest levels of drug loading (e.g. 25\%), there were few differences between them.

The present series of experiments contained within these four manuscripts show, therefore, that a proper evaluation of the materials produced by the wet agglomeration process must include an examination of each factor individually in terms of its effect on the product. The way in which each measured response is affected by changes in the level of the factor can be quantified and used to predict outcomes at other levels within the experimental range not actually tried. This is true for both complete optimization designs with independent variabels at several levels, as well as for simpler, two-level factorials. Sometimes more revealing, however, is the nature of the relationship between these factors, as depicted by interaction effects. 
APPENDIX II

CONCLUSIONS AND SUGGESTIONS FOR FUTURE WORK 


\section{General Conclusions}

1) The Double Planetary Mixer cannot be considered a high-speed or 'high-shear' mixer. Particle size analyses indicate that the agglomeration kinetics follow a pattern of unbridled growth uncontrolled by changes in mixing speed.

2) Over the entire range of available mixing speeds using the Double Planetary Mixer, the effect of mixing speed was usually not significant. It did, however, alter (interact with) some of the effects of water addition.

3) For the wet granulation process using the Double Planetary Mixer, the amount of water used in the process was the controlling factor, affecting nearly every parameter measured.

4) It was possible to construct, using response surface methodology, a map of those combinations of variables which lead to either an optimum product or a best compromise, based upon established criteria for multiple output variables.

5) A process optimum was located at a mixing speed of 60 RPM and $425 \mathrm{ml}$ of water addition. This was close to the suspected optimum of 67 RPM and $485 \mathrm{ml}$ of water, which was chosen as the center point of the central composite design.

6) The results obtained using optimization techniques can be used to locate a prospective 'end-point' which will yield the best possible product. Experimental results indicate an optimum set of conditions which do not coincide with either a direct-compression product or with traditional (subjective) means of end-point determination. The usual definition of 'end-point' should be adjusted to include conditions which objectively provide an optimum product. 
7) This study proposes the use of a new tableting parameter, the Hardness-Force Index (HFI), which combines the compactability and ejectability of tablets into one response. Its utility requires further study.

8) In Manuscript II, a complete factorial design was used to differentiate the abilities of the TK Fielder and the Ross PowerMix in a high-shear granulation process designed to fashion an acceptable acetaminophen product. The Ross PowerMix was shown to be more effective as a high-speed mixer, based upon its ability to more substantially affect those properties shown experimentally to be functions of mixing speed.

9) All responses measured for granulations, tablets and tableting process parameters were significantly affected by changes in mixing speed. Almost without exception, higher speeds improved product quality.

10) It was not possible to produce, using only a binary system consisting of $10 \%$ pregelatinized starch and $90 \%$ acetaminophen, an acceptable directly-compressible product. Nevertheless, significant differences between starches indicate that Starch 1500 may be superior to Starch 1551 under experimental conditions.

11) In Manuscipt III it was possible to clarify the relative effects of mixing speed, the amount of water used, the type of starch used, and the method of drying on a process for producing a directly-compressible acetaminophen-starch powder using the Ross PowerMix. Of the four experimental variables, the speed of mixing was the most significant factor affecting product characteristics. In nearly every case, increases in mixing speed improved product quality. The amount of water used in the process was only slightly less important than mixing speed and had similar 
beneficial effects. The method of drying granulations had little effect. Product quality was determined chiefly prior to the drying stage of the process.

12) Experimental results indicate that Starch 1500 was associated with better tableting and flow properties than Starch 1551.

13) Despite marked differences in particle size and morphology, all three grades of Avicel $\circledast$ microcrystalline celluloses yielded acceptable spherical pellets during a fluidized-bed rotary granulation process. Larger grades PH-102 and PH-200 were judged better when more 'difficult', smaller-sized grades of theophylline were used.

14) The potential for spheronization using a binary system of anhydrous theophylline and microcrystalline cellulose is determined primarily by the grade of theophylline used. Smaller-sized and micronized grades were less amenable to a fluidized-bed rotary granulation technique.

15) It was more difficult to spheronize theophylline-MCC combinations as the proportion of drug increased. It was only possible to produce spheres with all possible combinations of raw material grades in potencies of $50 \%$ or less. When pellets could be produced with drug potencies up to $90 \%$, there was a marked decrease in sphericity if the proportion of drug exceeded $70 \%$.

16) In summary, it can be said that the methods used in this work, those of evaluating the physical characteristics of the granulations and tablets made therefrom, can be effective means for isolating and quantifying important formulation and process variables, classifying mixer types, and can be used to locate optimum process 
conditions. These methods provide rational, objective and scientific means for better defining important aspects of the wet granulation process.

Suggestions for Future Work:

1) To redefine current concepts of wet granulation in terms of end-point detection to include those situations which cannot be considered either direct-compression or do not conform to the usual definition of 'end-point'. A definition which is based upon actual optimum results should challenge traditional techniques of end-point detection.

2) Explore the utility of a single parameter such the Hardness-Force Index (HFI) for the simultaneous evaluation of the entire compression-ejection event. Although the mechanistic aspects of compaction are of great importance, the availability of a rapid, practical means of estimating the performance of a material undergoing the tableting process might be of great utility to the practicing formulator.

3) To develop a better technique for the analysis of pellets (spheres) using both standard techniques as well as image analysis. The fractal nature of the sample images makes some image analysis techniques more difficult to interpret.

4) To optimize the pelletization procedure using the rotary fluidized-bed insert. The confluence of forces which results in the agglomeration and spheronization of materials has not been adequately defined. As a result, process control limits the use of this rapid technique.

5) To more clearly define those properties of materials which might optimize the spheronization of drug-excipient blends. Special 'spheronization grades' might be developed. 


\section{APPENDIX III}

SUMMARY OF STATISTICS 


\section{Manuscript I}

Granulation Geometric Mean Partricle Size (microns) $d_{g e o}$

Response Surface Regression

\begin{tabular}{|l|c|}
\hline Response Mean & $559 \mu$ \\
\hline R-Square & 0.970 \\
\hline Lack of Fit? & No \\
\hline Stationary Point & Saddle Point \\
\hline
\end{tabular}

Backward Elimination Regression Results $\quad$ (SLSTAY=0.10)

\begin{tabular}{|l|c|c|c|c|c|}
\hline & df & SS & MS & F & Prob $>$ F \\
\hline Regression & 2 & 449525 & 224762 & 237.4 & 0.0001 \\
\hline Error & 16 & 15149 & 946 & & \\
\hline Total & 18 & 464764 & & & \\
\hline & & & & & \\
\hline Variable & $\begin{array}{c}\text { Parameter } \\
\text { Estimate }\end{array}$ & Std. Error & Type II SS & F & Prob>F \\
\hline INTERCEPT & 111 & 22.6 & 22930 & 24.22 & 0.0002 \\
\hline SPEED & - & - & - & - & - \\
\hline VOLUME & - & - & - & - & - \\
\hline SPD2 & - & - & - & - & - \\
\hline VOL $^{2}$ & 0.001226 & 0.000103 & 135102 & 142.7 & 0.0001 \\
\hline SPD•VOL $^{2}$ & 0.00510 & 0.000863 & 33090 & 34.95 & 0.0001 \\
\hline
\end{tabular}

[(-) indicates an insignificant term has been eliminated from the model] 


\section{ManuscriptI}

Surface-Volume Mean Particle Size $d_{v s}$

Response Surface Regression

\begin{tabular}{|l|c|}
\hline Response Mean & 926.7 \\
\hline R-Square & 0.913 \\
\hline Lack of Fit? & No \\
\hline Stationary Point & Saddle Point \\
\hline
\end{tabular}

Backward Elimination Regression Results $\quad($ SLSTAY=0.10)

\begin{tabular}{|l|c|c|c|c|c|}
\hline & df & SS & MS & F & Prob $>$ F \\
\hline Regression & 3 & 327621 & 109207 & 52.22 & 0.0001 \\
\hline Error & 15 & 31366 & 2091 & & \\
\hline Total & 18 & 358988 & & & \\
\hline & & & & & \\
\hline Variable & $\begin{array}{c}\text { Parameter } \\
\text { Estimate }\end{array}$ & Std. Error & Type II SS & F & Prob>F \\
\hline INTERCEPT & 248.2 & 112.8 & 10127 & 4.84 & 0.0438 \\
\hline SPEED & 9.341 & 3.327 & 16484 & 7.88 & 0.0133 \\
\hline VOLUME & - & - & - & - & - \\
\hline SPD & - & - & - & - & - \\
\hline VOL $^{2}$ & 0.00220 & 0.000466 & 46794 & 22.38 & 0.0003 \\
\hline SPD॰VOL & -0.0144 & 0.00693 & 8997 & 4.30 & 0.0557 \\
\hline
\end{tabular}

$[(-)$ indicates an insignificant term has been eliminated from the model] 


\section{Manuscript I}

Compressibility Index (\%)

Response Surface Regression

\begin{tabular}{|l|c|}
\hline Response Mean & 8.962 \\
\hline R-Square & 0.752 \\
\hline Lack of Fit? & No \\
\hline Stationary Point & Maximum \\
\hline
\end{tabular}

Backward Elimination Regression Results $\quad$ (SLSTAY=0.10)

\begin{tabular}{|l|c|c|c|c|c|}
\hline & df & SS & MS & F & Prob $>$ F \\
\hline Regression & 1 & 54.894 & 54.894 & 25.95 & 0.0001 \\
\hline Error & 17 & 27.501 & 2.115 & & \\
\hline Total & 18 & 82.395 & & & \\
\hline & & & & & \\
\hline Variable & $\begin{array}{c}\text { Parameter } \\
\text { Estimate }\end{array}$ & Std. Error & Type II SS & F & Prob>F \\
\hline INTERCEPT & 13.7338 & 1.00921 & 391.75 & 185.19 & 0.0001 \\
\hline SPEED & - & - & - & - & - \\
\hline VOLUME & - & - & - & - & - \\
\hline SPD & - & - & - & - & - \\
\hline VOL 2 & -0.00002054 & 0.00000403 & 54.894 & 25.95 & 0.0002 \\
\hline SPD·VOL & - & - & - & - & - \\
\hline
\end{tabular}

[(-) indicates an insignificant term has been eliminated from the model] 


\section{Manuscript I}

Particle Flow Rate (g/s)

Response Surface Regression

\begin{tabular}{|l|c|}
\hline Response Mean & 2.688 \\
\hline R-Square & 0.778 \\
\hline Lack of Fit? & No \\
\hline Stationary Point & Maximum \\
\hline
\end{tabular}

Backward Elimination Regression Results $\quad($ SLSTAY $=0.10)$

\begin{tabular}{|l|c|c|c|c|c|}
\hline & df & SS & MS & F & Prob>F \\
\hline Regression & 1 & 11.217 & 11.217 & 41.92 & 0.0001 \\
\hline Error & 17 & 4.549 & 0.2676 & & \\
\hline Total & 18 & 15.7667 & & & \\
\hline & & & & & \\
\hline Variable & $\begin{array}{c}\text { Parameter } \\
\text { Estimate }\end{array}$ & Std. Error & Type II SS & F & Prob>F \\
\hline INTERCEPT & 4.6662 & 0.3277 & 54.253 & 202.73 & 0.0001 \\
\hline SPEED & - & - & - & - & - \\
\hline VOLUME & - & - & - & - & - \\
\hline SPD $^{2}$ & - & - & - & - & - \\
\hline VOL $^{2}$ & -0.00000844 & 0.0000013 & 11.217 & 41.92 & 0.0001 \\
\hline SPD•VOL & - & - & - & - & - \\
\hline
\end{tabular}

[(-) indicates an insignificant term has been eliminated from the model] 


\section{Manuscript I}

Tablet Thickness Variation (RSD,\%)

Response Surface Regression

\begin{tabular}{|l|c|}
\hline Response Mean & 0.4085 \\
\hline R-Square & 0.488 \\
\hline Lack of Fit? & No \\
\hline Stationary Point & Saddle Point \\
\hline
\end{tabular}

Backward Elimination Regression Results $\quad($ SLSTAY $=0.10)$

\begin{tabular}{|c|c|c|c|c|c|}
\hline & df & SS & MS & $\mathrm{F}$ & Prob $>F$ \\
\hline Regression & 1 & 0.7467 & 0.7467 & 10.73 & 0.0045 \\
\hline Error & 17 & 1.831 & 0.06960 & & \\
\hline Total & 18 & 1.930 & & & \\
\hline Variable & $\begin{array}{c}\text { Parameter } \\
\text { Estimate } \\
\end{array}$ & Std. Error & Type II SS & F & Prob $>F$ \\
\hline INTERCEPT & -0.10175 & 0.1671 & 0.0258 & 0.37 & 0.5507 \\
\hline SPEED & - & - & - & - & - \\
\hline VOLUME & - & - & - & - & - \\
\hline $\mathrm{SPD}^{2}$ & - & - & - & - & - \\
\hline $\mathrm{VOL}^{2}$ & 0.00000218 & 0.00000066 & 0.7467 & 10.73 & 0.0045 \\
\hline SPD•VOL & - & - & - & - & - \\
\hline
\end{tabular}

[(-) indicates an insignificant term has been eliminated from the model] 


\section{Manuscript I}

Compression Peak Force (kN) Variability, reported as RSD (\%)

Response Surface Regression

\begin{tabular}{|l|c|}
\hline Response Mean & 3.747 \\
\hline R-Square & 0.594 \\
\hline Lack of Fit? & No \\
\hline Stationary Point & Saddle Point \\
\hline
\end{tabular}

Backward Elimination Regression Results $\quad$ (SLSTAY=0.10)

\begin{tabular}{|c|c|c|c|c|c|}
\hline & $\mathrm{df}$ & SS & MS & F & Prob $>F$ \\
\hline Regression & 2 & 167.86 & 83.93 & 37.32 & 0.0001 \\
\hline Error & 56 & 125.9 & 2.249 & & \\
\hline Total & 58 & 293.801 & & & \\
\hline Variable & $\begin{array}{l}\text { Parameter } \\
\text { Estimate }\end{array}$ & Std. Error & Type II SS & $\mathrm{F}$ & Prob $>F$ \\
\hline INTERCEPT & -0.780 & 0.6210 & 3.548 & 1.58 & 0.2143 \\
\hline SPEED & - & - & - & - & - \\
\hline VOLUME & - & - & - & - & - \\
\hline $\mathrm{SPD}^{2}$ & -0.0008178 & 0.000150 & 66.90 & 29.75 & 0.0001 \\
\hline $\mathrm{VOL}^{2}$ & - & - & - & - & - \\
\hline SPD•VOL & 0.000263 & 0.00003164 & 155.927 & 69.33 & 0.0001 \\
\hline
\end{tabular}

[(-) indicates an insignificant term has been eliminated from the model] 


\section{Manuscript I}

Tablet Cohesion Index $(\mathrm{kg} / \mathrm{kN})$

Response Surface Regression

\begin{tabular}{|l|c|}
\hline Response Mean & 1.430 \\
\hline R-Square & 0.602 \\
\hline Lack of Fit? & No \\
\hline Stationary Point & Saddle Point \\
\hline
\end{tabular}

Backward Elimination Regression Results $\quad($ SLSTAY $=0.10$ )

\begin{tabular}{|l|c|c|c|c|c|}
\hline & df & SS & MS & F & Prob>F \\
\hline Regression & 2 & 0.0726 & 0.0363 & 6.82 & 0.0151 \\
\hline Error & 16 & 0.1055 & 0.00659 & & \\
\hline Total & 18 & 0.1781 & & & \\
\hline & & & & & \\
\hline Variable & $\begin{array}{c}\text { Parameter } \\
\text { Estimate }\end{array}$ & Std. Error & Type II SS & F & Prob>F \\
\hline INTERCEPT & 2.432 & 0.3077 & 0.4118 & 62.45 & 0.0001 \\
\hline SPEED & - & - & - & - & - \\
\hline VOLUME & -0.00439 & 0.0725 & 13.62 & 13.62 & 0.002 \\
\hline SPD & - & - & - & - & - \\
\hline VOL $^{2}$ & 0.00000461 & 0.00000140 & 0.07161 & 13.48 & 0.0021 \\
\hline SPD॰VOL & - & - & - & - & - \\
\hline
\end{tabular}

[(-) indicates an insignificant term has been eliminated from the model] 


\section{Manuscript I}

Tablet Ejection Force Index (N/kg)

Response Surface Regression

\begin{tabular}{|l|c|}
\hline Response Mean & 1978 \\
\hline R-Square & 0.578 \\
\hline Lack of Fit? & No \\
\hline Stationary Point & Minimum \\
\hline
\end{tabular}

Backward Elimination Regression Results $\quad$ (SLSTAY $=0.10$ )

\begin{tabular}{|l|c|c|c|c|c|}
\hline & df & SS & MS & F & Prob $>$ F \\
\hline Regression & 1 & 408011 & 408011 & 16.57 & 0.0008 \\
\hline Error & 17 & 418621 & 24624 & & \\
\hline Total & 18 & 826632 & & & \\
\hline & & & & & \\
\hline Variable & $\begin{array}{c}\text { Parameter } \\
\text { Estimate }\end{array}$ & Std. Error & Type II SS & F & Prob>F \\
\hline INTERCEPT & 2.432 & 0.3077 & 0.4118 & 62.45 & 0.0001 \\
\hline SPEED & - & - & - & - & - \\
\hline VOLUME & -1.5221 & 0.3739 & 408011 & 16.57 & 0.0008 \\
\hline SPD & - & - & - & - & - \\
\hline VOL $^{2}$ & - & - & - & - & - \\
\hline SPD•VOL & - & - & - & - & - \\
\hline
\end{tabular}

[(-) indicates an insignificant term has been eliminated from the model] 
ManuscriptI

Hardness-Force Index (HFI) $\mathrm{kg}^{2} \mathrm{kN}^{-2} \mathrm{msec}^{2}$

Response Surface Regression

\begin{tabular}{|l|c|}
\hline Response Mean & 1.740 \\
\hline R-Square & 0.559 \\
\hline Lack of Fit? & No \\
\hline Stationary Point & Maximum \\
\hline
\end{tabular}

Backward Elimination Regression Results $\quad($ SLSTAY $=0.10$ )

\begin{tabular}{|l|c|c|c|c|c|}
\hline & df & SS & MS & F & Prob>F \\
\hline Regression & 2 & 0.7809 & 0.3904 & 6.80 & 0.0073 \\
\hline Error & 16 & 0.9185 & 0.0574 & & \\
\hline Total & 18 & 1.6994 & & & \\
\hline & & & & & \\
\hline Variable & $\begin{array}{c}\text { Parameter } \\
\text { Estimate }\end{array}$ & Std. Error & Type II SS & F & Prob>F \\
\hline INTERCEPT & -1.264 & 0.9079 & 0.1113 & 1.94 & 0.1829 \\
\hline SPEED & - & - & - & - & - \\
\hline VOLUME & 0.0118 & 0.00390 & 0.5240 & 9.13 & 0.0081 \\
\hline SPD2 & - & - & - & - & - \\
\hline VOL 2 & -0.00001105 & 0.00000413 & 0.4121 & 7.18 & 0.0165 \\
\hline SPD॰VOL & - & - & - & - & - \\
\hline
\end{tabular}

[(-) indicates an insignificant term has been eliminated from the model] 


\section{Manuscript I}

Tablet Disintegration Time (min)

Response Surface Regression

\begin{tabular}{|l|c|}
\hline Response Mean & 3.074 \\
\hline R-Square & 0.3356 \\
\hline Lack of Fit? & No \\
\hline Stationary Point & Minimum \\
\hline
\end{tabular}

Backward Elimination Regression Results $\quad$ (SLSTAY=0.10)

\begin{tabular}{|l|c|c|c|c|c|}
\hline & df & SS & MS & F & Prob>F \\
\hline Regression & 1 & 2.5899 & 2.5899 & 4.52 & 0.0485 \\
\hline Error & 17 & 9.742 & 0.5730 & & \\
\hline Total & 18 & 12.332 & & & \\
\hline & & & & & \\
\hline Variable & $\begin{array}{c}\text { Parameter } \\
\text { Estimate }\end{array}$ & Std. Error & Type II SS & F & Prob>F \\
\hline INTERCEPT & 2.1238 & 0.4796 & 11.239 & 19.61 & 0.0004 \\
\hline SPEED & - & - & - & - & - \\
\hline VOLUME & - & - & - & - & - \\
\hline SPD $^{2}$ & - & - & - & - & - \\
\hline VOL $^{2}$ & 0.00000405 & 0.00000191 & 2.5899 & 4.52 & 0.0485 \\
\hline SPD॰VOL & - & - & - & - & - \\
\hline
\end{tabular}

[(-) indicates an insignificant term has been eliminated from the model] 


\section{Manuscript II}

Surface-Volume Mean Particle Size $d_{v s}$ (microns)

\begin{tabular}{|l|c|}
\hline Response Mean & 553.44 \\
\hline R-Square & 0.996 \\
\hline
\end{tabular}

Backward Elimination Regression Results $\quad($ SLSTAY $=0.10)$

\begin{tabular}{|l|c|c|c|c|c|}
\hline & df & SS & MS & F & Prob $>$ F \\
\hline Regression & 4 & 114353 & 28588 & 173.34 & 0.0007 \\
\hline Error & 3 & 494.8 & 164.9 & & \\
\hline Total & 7 & 114848 & & & \\
\hline & & & & & \\
\hline Variable & $\begin{array}{c}\text { Parameter } \\
\text { Estimate }\end{array}$ & Std. Error & Type II SS & F & Prob $>$ F \\
\hline INTERCEPT & 553.44 & 4.54 & 121.89 & & 0.0001 \\
\hline STARCH & -16.39 & 4.54 & -3.609 & 13.03 & 0.0365 \\
\hline MIXER & -73.56 & 4.54 & -16.202 & 262.49 & 0.0005 \\
\hline SPEED & -88.01 & 4.54 & -19.384 & 375.75 & 0.0003 \\
\hline MIXER•SPEED & -29.46 & 4.54 & -6.489 & 42.11 & 0.0074 \\
\hline STARCH•SPEED & - & - & - & - & - \\
\hline
\end{tabular}

[(-) indicates an insignificant term has been eliminated from the model] 


\section{Manuscript II}

Geometric Mean Particle Size Standard Deviation $\sigma_{8 e \infty}$ (Particle Size Uniformity)

\begin{tabular}{|l|c|}
\hline Response Mean & 2.275 \\
\hline R-Square & 0.9349 \\
\hline
\end{tabular}

Backward Elimination Regression Results $\quad$ (SLSTAY=0.10)

\begin{tabular}{|l|c|c|c|c|c|}
\hline & df & SS & MS & F & Prob $>$ F \\
\hline Regression & 5 & 1.385 & 0.2771 & 5.75 & 0.155 \\
\hline Error & 2 & 0.096 & 0.048 & & \\
\hline Total & 7 & 1.4822 & & & \\
\hline & & & & & \\
\hline Variable & $\begin{array}{c}\text { Parameter } \\
\text { Estimate }\end{array}$ & Std. Error & Type II SS & F & Prob $>$ F \\
\hline INTERCEPT & 2.2753 & 0.1063 & 41.414 & 457.42 & 0.0001 \\
\hline STARCH & - & - & - & - & - \\
\hline MIXER & -0.279 & 0.1063 & 0.6227 & 6.80 & 0.0469 \\
\hline SPEED & -0.2255 & 0.1063 & 0.4068 & 4.49 & 0.0875 \\
\hline MIXER•SPEED & - & - & - & - & - \\
\hline STARCH•SPEED & - & - & - & - & - \\
\hline
\end{tabular}

[(-) indicates an insignificant term has been eliminated from the model] 


\section{Manuscript II}

Granulation Tapped Bulk Density (gm/cc)

\begin{tabular}{|l|c|}
\hline Response Mean & 0.6643 \\
\hline R-Square & 0.971 \\
\hline
\end{tabular}

Backward Elimination Regression Results $\quad($ SLSTAY $=0.10$ )

\begin{tabular}{|l|c|c|c|c|c|}
\hline & df & SS & MS & F & Prob>F \\
\hline Regression & 3 & 0.00857 & 0.00285 & 19.02 & 0.0079 \\
\hline Error & 4 & 0.000601 & 0.000150 & & \\
\hline Total & 7 & 0.009176 & & & \\
\hline & & & & & \\
\hline Variable & $\begin{array}{c}\text { Parameter } \\
\text { Estimate }\end{array}$ & Std. Error & Type II SS & F & Prob>F \\
\hline INTERCEPT & 0.6642 & 0.00433 & 3.5298 & 23493 & 0.0001 \\
\hline STARCH & - & - & - & - & - \\
\hline MIXER & -0.0195 & 0.00433 & 0.00304 & 20.25 & 0.0108 \\
\hline SPEED & -0.0240 & 0.00433 & 0.00461 & 30.67 & 0.0052 \\
\hline MIXER•SPEED & - & - & - & - & - \\
\hline STARCH•MIXER & 0.01075 & 0.00433 & 0.00092 & 6.15 & 0.0682 \\
\hline
\end{tabular}

[(-) indicates an insignificant term has been eliminated from the model] 


\section{Manuscript II}

Compressibility Index (\%)

\begin{tabular}{|l|l|}
\hline Response Mean & 23.63 \\
\hline R-Square & 0.843 \\
\hline
\end{tabular}

Backward Elimination Regression Results $\quad($ SLSTAY $=0.10$ )

\begin{tabular}{|l|c|c|c|c|c|}
\hline & df & SS & MS & F & Prob>F \\
\hline Regression & 3 & 113.75 & 37.917 & 7.21 & 0.0432 \\
\hline Error & 4 & 21.045 & 5.261 & & \\
\hline Total & 7 & 134.80 & & & \\
\hline & & & & & \\
\hline Variable & $\begin{array}{c}\text { Parameter } \\
\text { Estimate }\end{array}$ & Std. Error & Type II SS & F & Prob>F \\
\hline INTERCEPT & 23.462 & 0.8109 & 4403 & 837.05 & 0.0001 \\
\hline STARCH & 2.3875 & 0.8109 & 45.60 & 8.67 & 0.0422 \\
\hline MIXER & -2.1375 & 0.8109 & 36.55 & 6.95 & 0.0578 \\
\hline SPEED & -1.987 & 0.8109 & 31.601 & 6.01 & 0.0704 \\
\hline MIXER•SPEED & - & - & - & - & - \\
\hline STARCH•MIXER & - & - & - & - & - \\
\hline
\end{tabular}

[(-) indicates an insignificant term has been eliminated from the model] 


\section{Manuscript II}

Angle of Repose (degrees)

\begin{tabular}{|l|c|}
\hline Response Mean & 38.48 \\
\hline R-Square & 0.9034 \\
\hline
\end{tabular}

Backward Elimination Regression Results $\quad($ SLSTAY $=0.10)$

\begin{tabular}{|l|c|c|c|c|c|}
\hline & df & SS & MS & F & Prob $>$ F \\
\hline Regression & 5 & 167.36 & 33.47 & 33.70 & 0.0001 \\
\hline Error & 18 & 17.876 & 0.993 & & \\
\hline Total & 23 & 185.23 & & & \\
\hline & & & & & \\
\hline Variable & $\begin{array}{c}\text { Parameter } \\
\text { Estimate }\end{array}$ & Std. Error & Type II SS & F & Prob $>$ F \\
\hline INTERCEPT & 38.482 & 0.2034 & 35541 & 35788 & 0.0001 \\
\hline STARCH & 1.495 & 0.2034 & 53.70 & 54.07 & 0.0001 \\
\hline MIXER & -1.341 & 0.2034 & 43.15 & 43.45 & 0.0001 \\
\hline SPEED & -1.261 & 0.2034 & 38.15 & 38.42 & 0.0001 \\
\hline MIXER•SPEED & 1.059 & 0.2034 & 26.92 & 27.11 & 0.0001 \\
\hline STARCH•MIXER & -0.476 & 0.2034 & 5.434 & 5.47 & 0.0311 \\
\hline
\end{tabular}

$[(-)$ indicates an insignificant term has been eliminated from the model] 


\section{Manuscript II}

Tablet Compression Peak Force Variability (RSD,\%)

\begin{tabular}{|l|c|}
\hline Response Mean & 4.314 \\
\hline R-Square & 0.725 \\
\hline
\end{tabular}

Backward Elimination Regression Results $\quad($ SLSTAY $=0.10)$

\begin{tabular}{|l|c|c|c|c|c|}
\hline & df & SS & MS & F & Prob $>$ F \\
\hline Regression & 3 & 70.04 & 23.34 & 18.49 & 0.0001 \\
\hline Error & 21 & 26.517 & 1.263 & & \\
\hline Total & 24 & 96.56 & & & \\
\hline & & & & & \\
\hline Variable & $\begin{array}{c}\text { Parameter } \\
\text { Estimate }\end{array}$ & Std. Error & Type II SS & F & Prob $>$ F \\
\hline INTERCEPT & 4.314 & 0.2252 & 463.32 & 366.92 & 0.0001 \\
\hline STARCH & 0.543 & 0.2252 & 7.336 & 5.81 & 0.0252 \\
\hline MIXER & -0.582 & 0.2252 & 8.432 & 6.68 & 0.0173 \\
\hline SPEED & -1.484 & 0.2252 & 54.79 & 43.39 & 0.0001 \\
\hline MIXER-SPEED & - & - & - & - & - \\
\hline STARCH•SPEED & - & - & - & - & - \\
\hline
\end{tabular}

[(-) indicates an insignificant term has been eliminated from the model] 


\section{Manuscript II}

Tablet Cohesion Index $(\mathrm{kg} / \mathrm{kN})$

\begin{tabular}{|l|c|}
\hline Response Mean & 0.1794 \\
\hline R-Square & 0.864 \\
\hline
\end{tabular}

Backward Elimination Regression Results $\quad$ (SLSTAY $=0.10)$

\begin{tabular}{|l|c|c|c|c|c|}
\hline & df & SS & MS & F & Prob $>$ F \\
\hline Regression & 5 & 0.2589 & 0.0517 & 94.45 & 0.0001 \\
\hline Error & 74 & 0.0406 & 0.000548 & & \\
\hline Total & 79 & 0.2995 & & & \\
\hline & & & & & \\
\hline Variable & $\begin{array}{c}\text { Parameter } \\
\text { Estimate }\end{array}$ & Std. Error & Type II SS & F & Prob $>$ F \\
\hline INTERCEPT & 0.1794 & 0.0026 & 2.576 & 4697 & 0.0001 \\
\hline STARCH & -0.02707 & 0.0026 & 0.0586 & 106.94 & 0.0001 \\
\hline MIXER & 0.0064 & 0.0026 & 0.00327 & 5.98 & 0.0169 \\
\hline SPEED & 0.04865 & 0.0026 & 0.1893 & 345.28 & 0.0001 \\
\hline MIXER•SPEED & -0.00505 & 0.0026 & 0.00204 & 3.72 & 0.0576 \\
\hline STARCH•SPEED & 0.00842 & 0.0026 & 0.00567 & 10.35 & 0.0019 \\
\hline
\end{tabular}

[(-) indicates an insignificant term has been eliminated from the model] 
Manuscript II

Tablet Ejection Force Index (N/kg)

\begin{tabular}{|l|c|}
\hline Response Mean & 133.70 \\
\hline R-Square & 0.800 \\
\hline
\end{tabular}

Backward Elimination Regression Results $\quad($ SLSTAY $=0.10)$

\begin{tabular}{|l|c|c|c|c|c|}
\hline & df & SS & MS & F & Prob $>$ F \\
\hline Regression & 3 & 70445 & 23481 & 101.44 & 0.0001 \\
\hline Error & 76 & 17592 & 231.5 & & \\
\hline Total & 79 & 88037 & & & \\
\hline & & & & & \\
\hline Variable & $\begin{array}{c}\text { Parameter } \\
\text { Estimate }\end{array}$ & Std. Error & Type II SS & F & Prob $>$ F \\
\hline INTERCEPT & 133.698 & 1.7010 & 1430023 & 6177 & 0.0001 \\
\hline STARCH & 8.115 & 1.7010 & 5268 & 22.76 & 0.0001 \\
\hline MIXER & 21.592 & 1.7010 & 37297 & 161.12 & 0.0001 \\
\hline SPEED & -18.668 & 1.7010 & 27879 & 120.44 & 0.0001 \\
\hline MIXER-SPEED & - & - & - & - & - \\
\hline STARCH•SPEED & - & - & - & - & - \\
\hline
\end{tabular}

[(-) indicates an insignificant term has been eliminated from the model] 
Manuscript II

Tablet Disintegration Time (sec)

\begin{tabular}{|l|c|}
\hline Response Mean & 23.62 \\
\hline R-Square & 0.9807 \\
\hline
\end{tabular}

Backward Elimination Regression Results $\quad($ SLSTAY $=0.10)$

\begin{tabular}{|l|c|c|c|c|c|}
\hline & df & SS & MS & F & Prob $>$ F \\
\hline Regression & 6 & 10054 & 1675 & 348.85 & 0.0001 \\
\hline Error & 41 & 196.95 & 4.803 & & \\
\hline Total & 47 & 10251 & & & \\
\hline & & & & & \\
\hline Variable & $\begin{array}{c}\text { Parameter } \\
\text { Estimate }\end{array}$ & Std. Error & Type II SS & T for $\mathrm{H}_{0}>0$ & Prob>F \\
\hline INTERCEPT & 23.617 & 0.3163 & 26771 & 5573 & 0.0001 \\
\hline STARCH & -5.458 & 0.3163 & 1430 & 297.7 & 0.0001 \\
\hline MIXER & 7.383 & 0.3163 & 2617 & 544.7 & 0.0001 \\
\hline SPEED & 7.417 & 0.3163 & 2640 & 549.7 & 0.0001 \\
\hline STARCH•MIXER & -4.208 & 0.3163 & 850 & 177.0 & 0.0001 \\
\hline MIXXR•SPEED & 6.250 & 0.3163 & 1875 & 390.3 & 0.0001 \\
\hline STARCH•SPEED & -3.658 & 0.3163 & 642 & 133.7 & 0.0001 \\
\hline
\end{tabular}

[(-) indicates an insignificant term has been eliminated from the model] 


\section{Manuscript II}

Tablet Friability (\%)

\begin{tabular}{|l|c|}
\hline Response Mean & 7.21 \\
\hline R-Square & 0.9566 \\
\hline
\end{tabular}

Backward Elimination Regression Results $\quad($ SLSTAY $=0.10)$

\begin{tabular}{|l|c|c|c|c|c|}
\hline & df & SS & MS & F & Prob>F \\
\hline Regression & 4 & 163.9 & 40.98 & 16.57 & 0.0219 \\
\hline Error & 3 & 7.42 & 2.473 & & \\
\hline Total & 7 & 171.32 & & & \\
\hline & & & & & \\
\hline Variable & $\begin{array}{c}\text { Parameter } \\
\text { Estimate }\end{array}$ & Std. Error & Type II SS & T for $\mathrm{H}_{0}>0$ & Prob>F \\
\hline INTERCEPT & 7.21 & 0.5560 & N/A & 12.968 & 0.0010 \\
\hline STARCH & 1.695 & 0.5560 & N/A & 3.049 & 0.0555 \\
\hline MIXER & -1.5267 & 0.5560 & N/A & -2.746 & 0.0710 \\
\hline SPEED & - & - & N/A & - & - \\
\hline MIXER•SPEED & 0.483 & 0.5560 & N/A & 0.869 & 0.4490 \\
\hline STARCH•SPEED & - & - & N/A & - & - \\
\hline
\end{tabular}

[(-) indicates an insignificant term has been eliminated from the model] 
Manuscript III

Surface-Volume Mean Particle Size $d_{v s}$ (microns)

\begin{tabular}{|l|l|}
\hline Response Mean & 576.90 \\
\hline R-Square & 0.9288 \\
\hline
\end{tabular}

Backward Elimination Regression Results $\quad($ SLSTAY $=0.10)$

\begin{tabular}{|l|c|c|c|c|c|}
\hline & df & SS & MS & F & Prob>F \\
\hline Regression & 4 & 328988 & 82247 & 35.88 & 0.0001 \\
\hline Error & 11 & 25217 & 2292 & & \\
\hline Total & 15 & 354206 & & & \\
\hline & & & & & \\
\hline Variable & $\begin{array}{c}\text { Parameter } \\
\text { Estimate }\end{array}$ & Std. Error & Type II SS & F & Prob>F \\
\hline INTERCEPT & 576.90 & 11.97 & 5325017. & 2322.8 & 0.0001 \\
\hline STARCH & - & - & - & - & - \\
\hline SPEED & -106.98 & 11.97 & 183098 & 79.87 & 0.0001 \\
\hline VOLUME & 29.10 & 11.97 & 13548 & 5.910 & 0.0333 \\
\hline DRIER & 81.80 & 11.97 & 107059 & 46.70 & 0.0001 \\
\hline SPEED•WATER & 39.75 & 11.97 & 25281 & 11.30 & 0.0068 \\
\hline SPEED-STARCH & - & - & - & - & - \\
\hline
\end{tabular}

[(-) indicates an insignificant term has been eliminated from the model] 


\section{Manuscript III}

Granule Geometric Mean Particle Size Standard Deviation $\sigma_{\text {geo }}$ (Particle Size Uniformity)

\begin{tabular}{|l|l|}
\hline Response Mean & 1.897 \\
\hline R-Square & 0.826 \\
\hline
\end{tabular}

Backward Elimination Regression Results $\quad($ SLSTAY $=0.10)$

\begin{tabular}{|l|c|c|c|c|c|}
\hline & df & SS & MS & F & Prob $>$ F \\
\hline Regression & 2 & 0.6411 & 0.3206 & 30.84 & 0.0001 \\
\hline Error & 13 & 0.1351 & 0.0104 & & \\
\hline Total & 15 & 0.7762 & & & \\
\hline & & & & & \\
\hline Variable & $\begin{array}{c}\text { Parameter } \\
\text { Estimate }\end{array}$ & Std. Error & Type II SS & F & Prob>F \\
\hline INTERCEPT & 1.8969 & 0.0254 & 57.573 & 5539 & 0.0001 \\
\hline STARCH & - & - & - & - & - \\
\hline SPEED & -0.1896 & 0.0254 & 0.5757 & 55.39 & 0.0001 \\
\hline VOLUME & - & - & - & - & - \\
\hline DRIER & 0.0639 & 0.0254 & 0.0654 & 6.29 & 0.0262 \\
\hline SPEED•WATER & - & - & - & - & - \\
\hline SPEED•STARCH & - & - & - & - & - \\
\hline
\end{tabular}

[(-) indicates an insignificant term has been eliminated from the model] 
Manuscript III

Granulation Compressibility Index (\%)

\begin{tabular}{|l|c|}
\hline Response Mean & 19.306 \\
\hline R-Square & 0.595 \\
\hline
\end{tabular}

Backward Elimination Regression Results $\quad($ SLSTAY $=0.10)$

\begin{tabular}{|l|c|c|c|c|c|}
\hline & df & SS & MS & F & Prob>F \\
\hline Regression & 2 & 138.18 & 69.09 & 9.55 & 0.0028 \\
\hline Error & 13 & 94.03 & 7.232 & & \\
\hline Total & 15 & 232.21 & & & \\
\hline & & & & & \\
\hline Variable & $\begin{array}{c}\text { Parameter } \\
\text { Estimate }\end{array}$ & Std. Error & Type II SS & F & Prob>F \\
\hline INTERCEPT & 19.306 & 0.6723 & 5963 & 824.52 & 0.0001 \\
\hline STARCH & - & - & - & - & - \\
\hline SPEED & -1.756 & 0.6723 & 49.35 & 6.82 & 0.0215 \\
\hline VOLUME & -2.356 & 0.6723 & 88.83 & 12.28 & 0.0039 \\
\hline DRIER & - & - & - & - & - \\
\hline SPEED•WATER & - & - & - & - & - \\
\hline SPEED•STARCH & - & - & - & - & - \\
\hline
\end{tabular}

[(-) indicates an insignificant term has been eliminated from the model] 
Manuscript III

Angle of Repose (degrees)

\begin{tabular}{|l|c|}
\hline Response Mean & 36.412 \\
\hline R-Square & 0.517 \\
\hline
\end{tabular}

Backward Elimination Regression Results $\quad($ SLSTAY $=0.10)$

\begin{tabular}{|l|c|c|c|c|c|}
\hline & df & SS & MS & F & Prob $>$ F \\
\hline Regression & 2 & 4.10 & 11.275 & 11.53 & 0.0001 \\
\hline Error & 43 & 42.06 & 0.9782 & & \\
\hline Total & 47 & 87.16 & & & \\
\hline & & & & & \\
\hline Variable & $\begin{array}{c}\text { Parameter } \\
\text { Estimate }\end{array}$ & Std. Error & Type II SS & F & Prob>F \\
\hline INTERCEPT & 36.412 & 0.1427 & 63642 & 65055 & 0.0001 \\
\hline STARCH & 0.5160 & 0.1427 & 12.782 & 13.07 & 0.0008 \\
\hline SPEED & -0.3640 & 0.1427 & 6.358 & 6.50 & 0.0144 \\
\hline VOLUME & 0.5523 & 0.1427 & 14.64 & 14.97 & 0.0004 \\
\hline DRIER & - & - & - & - & - \\
\hline STARCH•WATER & -0.4855 & 0.1427 & 11.32 & 11.57 & 0.0015 \\
\hline SPEED-STARCH & - & - & - & - & - \\
\hline
\end{tabular}

$[(-)$ indicates an insignificant term has been eliminated from the model] 
Manuscript III

Tablet Cohesion Index $(\mathrm{kg} / \mathrm{kN})$

\begin{tabular}{|l|l|}
\hline Response Mean & 0.2055 \\
\hline R-Square & 0.7086 \\
\hline
\end{tabular}

Backward Elimination Regression Results $\quad$ (SLSTAY=0.10)

\begin{tabular}{|l|c|c|c|c|c|}
\hline & df & SS & MS & F & Prob>F \\
\hline Regression & 5 & 0.3779 & 0.0755 & 74.91 & 0.0001 \\
\hline Error & 154 & 0.1553 & 0.0010 & & \\
\hline Total & 159 & 0.5332 & & & \\
\hline & & & & & \\
\hline Variable & $\begin{array}{l}\text { Parameter } \\
\text { Estimate }\end{array}$ & Std. Error & Type II SS & F & Prob>F \\
\hline INTERCEPT & 0.20553 & 0.0025 & 6.759 & 6699 & 0.0001 \\
\hline STARCH & -0.0183 & 0.0025 & 0.0535 & 53.03 & 0.0001 \\
\hline SPEED & 0.03227 & 0.0025 & 0.1666 & 165.19 & 0.0001 \\
\hline VOLUME & 0.0263 & 0.0025 & 0.1111 & 110.2 & 0.0001 \\
\hline DRIER & - & - & - & - & - \\
\hline STARCH•WATER & 0.0060 & 0.0025 & 0.0058 & 5.78 & 0.0174 \\
\hline SPEED-STARCH & 0.0159 & 0.0025 & 0.0407 & 40.34 & 0.0001 \\
\hline
\end{tabular}

[(-) indicates an insignificant term has been eliminated from the model] 
Manuscript III

Tablet Ejection Force Index (N/kg)

\begin{tabular}{|l|c|}
\hline Response Mean & 362.98 \\
\hline R-Square & 0.8082 \\
\hline
\end{tabular}

Backward Elimination Regression Results $\quad($ SLSTAY $=0.10)$

\begin{tabular}{|l|c|c|c|c|c|}
\hline & df & SS & MS & F & Prob>F \\
\hline Regression & 4 & 28613 & 7153 & 11.59 & 0.0006 \\
\hline Error & 11 & 6788 & 617.2 & & \\
\hline Total & 15 & 35402 & & & \\
\hline & & & & & \\
\hline Variable & $\begin{array}{c}\text { Parameter } \\
\text { Estimate }\end{array}$ & Std. Error & Type II SS & F & Prob>F \\
\hline INTERCEPT & 362.975 & 6.211 & 2108013 & 3415.6 & 0.0001 \\
\hline STARCH & -23.68 & 6.211 & 8968.1 & 14.53 & 0.0029 \\
\hline SPEED & 26.89 & 6.11 & 11567 & 18.74 & 0.0012 \\
\hline VOLUME & 15.662 & 6.211 & 3925 & 6.36 & 0.0284 \\
\hline DRIER & -16.11 & 6.211 & 4153 & 6.73 & 0.0249 \\
\hline STARCH•WATER & - & - & - & - & - \\
\hline SPEED•STARCH & - & - & - & - & - \\
\hline
\end{tabular}

[(-) indicates an insignificant term has been eliminated from the model] 
Manuscript III

Tablet Disintegration Time (sec)

\begin{tabular}{|l|c|}
\hline Response Mean & 47.125 \\
\hline R-Square & 0.878 \\
\hline
\end{tabular}

Backward Elimination Regression Results $\quad($ SLSTAY $=0.10$ )

\begin{tabular}{|l|c|c|c|c|c|}
\hline & df & SS & MS & F & Prob>F \\
\hline Regression & 5 & 110796 & 22159 & 130.37 & 0.0001 \\
\hline Error & 90 & 15297 & 169.9 & & \\
\hline Total & 95 & 126094 & & & \\
\hline & & & & & \\
\hline Variable & $\begin{array}{c}\text { Parameter } \\
\text { Estimate }\end{array}$ & Std. Error & Type II SS & F & Prob>F \\
\hline INTERCEPT & 47.125 & 1.33 & 213193 & 1254.3 & 0.0001 \\
\hline STARCH & - & - & - & - & - \\
\hline SPEED & 25.17 & 1.33 & 60802 & 357.7 & 0.0001 \\
\hline VOLUME & 15.81 & 1.33 & 24003 & 141.2 & 0.0001 \\
\hline SPEED•WATER & 11.687 & 1.33 & 13113 & 77.15 & 0.0001 \\
\hline STARCH•WATER & 10.29 & 1.33 & 10168 & 59.82 & 0.0001 \\
\hline SPEED•STARCH & 5.312 & 1.33 & 2709 & 15.94 & 0.0001 \\
\hline
\end{tabular}

[( - ) indicates an insignificant term has been eliminated from the model] 


\section{Manuscript III}

Tablet Friability (\%)

\begin{tabular}{|l|l|}
\hline Response Mean & 6.968 \\
\hline R-Square & 0.784 \\
\hline
\end{tabular}

Backward Elimination Regression Results $\quad($ SLSTAY=0.10)

\begin{tabular}{|l|c|c|c|c|c|}
\hline & df & SS & MS & F & Prob $>F$ \\
\hline Regression & 5 & 620.1 & 124.0 & 18.93 & 0.0001 \\
\hline Error & 26 & 170.3 & 6.549 & & \\
\hline Total & 31 & 790.3 & & & \\
\hline & & & & & \\
\hline Variable & $\begin{array}{l}\text { Parameter } \\
\text { Estimate }\end{array}$ & Std. Error & Type II SS & F & Prob>F \\
\hline INTERCEPT & 6.968 & 0.4524 & 1553.8 & 237.24 & 0.0001 \\
\hline STARCH & 1.060 & 0.4524 & 35.93 & 5.49 & 0.0271 \\
\hline SPEED & -2.721 & 0.4524 & 236.85 & 36.16 & 0.0001 \\
\hline VOLUME & -1.695 & 0.4524 & 91.95 & 14.04 & 0.0009 \\
\hline COMP SETTING* & 2.6727 & 0.4524 & 228.59 & 34.90 & 0.0001 \\
\hline STARCH•WATER & -0.9134 & 0.4524 & 26.70 & 4.08 & 0.0539 \\
\hline SPEED•STARCH & - & - & - & - & - \\
\hline
\end{tabular}

* compression setting during tableting

[(-) indicates an insignificant term has been eliminated from the model] 


\section{APPENDIX IV}

PUBLICATIONS AND PRESENTATIONS 


\section{PUBLICATIONS}

Sienkiewicz G., Pereira R., Rudnic E. M.(1990) "Spheronization of Theophylline and Microcrystalline Cellulose Combinations Using a Fluidized-Bed Rotogranulator", Pharmaceutical Research 7(9): S-69.

Sienkiewicz G., Lausier J., Rhodes C.T.(1991) "Recent Advances in Pharmaceutical Mixing Technology", Proceedings of the 1991 Interphex Conference, 185-192.

Sienkiewicz G., Lausier J., Rhodes C.T.(1993) "An Investigation of a Wet Granulation Process Using a Double Planetary Mixer", Proceedings of the Interphex Conference, 239248.

Sienkiewicz G., Lausier J., Rhodes C.T.(1993) An Investigation of a Wet Granulation Process Using Two Mixers, Two Binders and Two Process Variables, Pharmaceutical Research 10(10):S167.

Sienkiewicz G. (1995) A Factorial Design Study of Two High-Shear Mixers and Two Starches, Proceedings of the Interphex Conference (submitted for publication January 1995). 


\section{PRESENTATIONS}

American Association of Pharmaceutical Scientists Conference Fifth Annual Meeting and Exposition, Las Vegas, NV (1990) "Spheronization of Theophylline and Microcrystalline Cellulose Combinations Using a Fluidized-Bed Rotogranulator".

Interphex - USA Conference and Exhibition 1991 (Podium Session) "Recent Advances in Pharmaceutical Mixing Technology".

Interphex - USA Conference and Exhibition 1993 (Podium Session), "An Investigation of a Wet Granulation Process Using a Double Planetary Mixer".

American Association of Pharmaceutical Scientists Conference Eighth Annual Meeting and Exposition, Orlando, FL (1993), "An Investigation of a Wet Granulation Process Using Two Mixers, Two Binders and Two Process Variables".

Interphex - USA Conference and Exhibition 1995 (Podium Session), "A Factorial Design Study of Two High-Shear Mixers and Two Starches" (accepted for presentation March $30,1995)$. 
BIBLIOGRAPHY 
Applegren C. (1985) Recent Advances in Granulation Technology and Equipment, Drug Development and Industrial Pharmacy, 11(2\&3), 725-741.

Augsburger L.L., Shangraw R.F. (1966) Effect of Glidants in Tableting, Journal of Pharmaceutical Sciences, 55(4), 418-423.

Avicel Spheres, FMC Corporation, Food and Pharmaceutical Products Division, Philadelphia.

Bataille B., Ligarski K., Jacob M., Thomas C., Duru C. (1993) Study of the Influence of Spheronization and Drying Conditions on the Physico-Mechanical Properties of Neutral Spheroids Containing Avicel PH-101 and Lactose, Drug Development and Industrial Pharmacy, 19(6), 653-671.

Bohidar N.R. (1991) Pharmaceutical Formulation Optimization Using SAS ${ }^{\mathrm{TM}}$, Drug Development and Industrial Pharmacy, 17(3), 421-441.

Box G.E.P. (1954) The Exploration and Exploitation of Response Surfaces: Some General Considerations and Examples, 10, 16-60.

Box G.E.P., Draper N. (1987) Empirical Model Building and Response Surfaces, John Wiley \& Sons, New York, p. 511.

Boylan J.C., Cooper J., Chowhan Z.T., Lund W., Wade A., Weir R.F., Yates B.J. (1986) Handbook of Pharmaceutical Excipients, American Pharmaceutical Association, Washington, D.C. 
Brittain H.G., Bogdanowich S.J., Bugay D.E., DeVincentis J., Lewen G., Newman A.W. (1991) Physical Characterization of Pharmaceutical Solids, Pharmaceutical Research, 8(8), 963-973.

Carr R.L. (1965) Evaluating Flow Properties of Solids, Chemical Engineering, 72, 163168.

Celik M. (1992) Overview of compaction Data Analysis Techniques, Drug Development and Industrial Pharmacy, 18(6\&7), 767-810.

Chichester E.H. (1988) Tablet Machine Instrumentation in Pharmaceutics: Principles and Practice, Halsted Press, New York.

Chowhan Z. (1993) Excipients and Their Functionality in Drug Product Development, Pharmaceutical Technology, 17(9),72-82.

Chowhan Z. (1994) Drug Substance Physical Properties and Their Relationship to the Performance of Solid Dosage Forms, Pharmaceutical Technology, 18(3),44-60.

Cliff M.J. (1990) Granulation End Point and Automated Process of Mixer-Granulators:Part I, Pharmaceutical Technology, 14(4),112-132.

Cliff M.J. (1990) Granulation End Point and Automated Process of Mixer-Granulators:Part II, Pharmaceutical Technology, 14(5),36-44. 
Corvari V., Fry W.C., Seibert W.L., Augsberger L. (1992) Instrumentation of a HighShear Mixer: Evaluation and Comparison of a New Capacitive Sensor, a Watt Meter, and a Strain-Gauge Torque Sensor for Wet Granulation Monitoring, Pharmaceutical Research, 9(12), 1525-1533.

Elbers J.A.C., Bakkenes H.W., Fokkens J.G. (1992) Effect of Amount and Composition of Granulation Liquid on Mixing, Extrusion and Spheronization, Drug Development and Industrial Pharmacy, 18(5), 501-517.

Finnan J.L., Lisa R.E., Schmidt D.N. (1987) Process for Preparing Spray Dried Acetaminophen Powder and the Powder Prepared Thereby, United States Patent $\# 4,710,519$.

Goskonda S.R., Upadrashta S.M. (1993) Avicel RC-591/Chitosan Beads by Extrusion/Spheronization Technology, Drug Development and Industrial Pharmacy, 19(8), 915-927.

Harris M.R., Schwartz J.B., McGinity J.W. (1985) Optimization of a Slow-Release Tablet Formulation Containing Sodium Sulfathiazole and Montmorillonite Clay, Drug Development and Industrial Pharmacy, 11(5), 1089-1110.

Hileman G.A., Goskonda S.R., Spallito A.J., Upadrashta S.M. (1993) A Factorial Approach to High Dose Product Development by an Extrusion/Spheronization Process, Drug Development and Industrial Pharmacy, 19(4), 483-491. 
Hines W.W., Montgomery D.C. (1990) Probability and Statistics in Engineering and Management Science, Third Edition, John Wiley \& Sons, New York.

Jäger K.F., Bauer K.H. (1982) Effect of Material Motion on Agglomeration in the Rotary Fluidized-Bed Granulator, Drugs Made in Germany, 25, 61-65.

Kottke M.K., Chueh H.R., Rhodes C.T. (1992) Comparison of Disintegrant and Binder Activity of Three Corn Starch Products, Drug Development and Industrial Pharmacy, 18(20), 2207-2223.

Kristensen H.G. (1988) Agglomeration of Powders, Acta Pharmaceutica Suecica, 25, 187204.

Kristensen H.G., Schaefer T. (1987) A Review of Pharmaceutical Wet-Granulation, Drug Development and Industrial Pharmacy, 13(4\&5), 812-813.

Lachman L., Lieberman H.A., Kanig J.L. (1986) The Theory and Practice of Industrial Pharmacy, Third Edition, Lea \& Febiger, Philadelphia.

Lerk I.C.F., Bolhuis G.K.De Boer A.H. (1974) Comparative Evaluation of Excipients for Direct Compression, Pharmaceutisch Weekblad, 40, 945-955.

Leuenberger H. (1982) Granulation, New Techniques, Pharmaceutica Acta Helvetica, 57(3), 72-81. 
Lieberman H.A.,Lachman L., Schwartz J.B. (1990) Pharmaceutical Dosage forms: Tablets, Volume 2, Second Edition, Marcel Dekker, New York.

Liu C.H., Chen S.C., Lee Y.C., Sokoloski T.D., Sheu M.T. (1994) Directly Compressible Acetaminophen Compositions Prepared by Fluidized-Bed Granulation, Drug Development and Industrial Pharmacy, 20(11), 1911-1922.

Malinowski H.J.,Smith W.E. (1975) Use of Factorial Design to Evaluate Granulations Prepared by Spheronization, Journal of Pharmaceutical Sciences, 64(10), 1688-1692.

Martin A., Swarbrick J., Cammarata A., Chun A.H.C. (1983) Physical Pharmacy, Third Edition, Lea \& Febiger, Philadelphia.

Montgomery D.C., Design and Analysis of Experiments (1991) Design and Analysis of Experiments, Third Edition, John Wiley \& Sons, New York.

Newitt D.M., Conway-Jones J.M. (1958), Transactions of the Institution of Chemical Engineers, 36, 422.

Newton J.M.,Chow A.K., Jeewa K.B. (1993) The Effect of Excipient Source on Spherical Granules Made by Extrusion/Spheronization, Pharmaceutical Technology, 17(3) 166-174.

Otsuka M., Gao J., Matsuda Y. (1994) Effect of Amount of Added Water During Extrusion-Spheronization Process on Pharmaceutical Properties of Granules, Drug Development and Industrial Pharmacy, 20(19), 2977-2992. 
Parrott E.L. (1970) Pharmaceutical Technology, Burgess Publishing Company, Minneapolis.

Patel N.K., Poola N.R., Babar A., Plakogiannis F.M. (1989) Fluidized-Bed Agglomeration of Acetaminophen; Direct Compression of Tablets and Physiologic Availability, Drug Development and Industrial Pharmacy, 15(8), 1175-1198.

Pendharkar C.M.,Jhawar R.J., Rutledge J.M., Hause W., Grim W.M., Harwood R.J. (1990) Influence of the Specific Surface Area of Selected Raw Materials on the Granulation Process Using an Instrumented Mixer, Pharmaceutical Technology, 14(4), 44-53.

Robinson R.L., Hollenbeck R.G. (1991) Manufacture of Spherical Acetaminophen Pellets:Comparison of Rotary Processing with Multiple-Step Extrusion and Spheronization, Pharmaceutical Technology, 15(5), 48-56.

Rumpf H. (1974), Chemical Ingineering Technologie, 46, 1.

Salpekar A.M., Denton L.E. (1987) Direct Tableting Acetaminophen Compositions, United States Patent \#4,661,521.

Salpekar A.M., Denton L.E. (1987) Direct Tableting Acetaminophen Compositions, United States Patent \#4,757,090.

Sanghvi P.P., Collins C.C., Shukla A.J. (1993) Evaluation of Preflo® Modified Starches as New Direct Compression Excipients. I. Tabletting Characteristics, Pharmaceutical Research, 10(11), 1597-1603. 
Schwartz J.B. (1981) Optimization Techniques in Product Formulation, Journal of the Society of Cosmetic Chemists, 32, 287-301.

Schwartz J.B. (1988) Granulation, Drug Development and Industrial Pharmacy, 14(14), 2071-2090.

Schwartz J.B., Flamholz J.R., Press R.H. (1973) Computer Optimization of Pharmaceutical Formulations I:General Procedure, Journal of Pharmaceutical Sciences, 62(7), 1165-1170.

Schwartz J.B., Flamholz J.R., Press R.H. (1973) Computer Optimization of Pharmaceutical Formulations II:Application in Troubleshooting, Journal of Pharmaceutical Sciences, 62(7), 1165-1170.

Shangraw R.F., Wallace J.W., Bowers F.M. (1981) Morphology and Functionality in Tablet Excipients for Direct Compression: Part I, Pharmaceutical Technology, 10, 1-10.

Shangraw R.F., Wallace J.W., Bowers F.M. (1981) Morphology and Functionality in Tablet Excipients for Direct Compression: Part II, Pharmaceutical Technology, 10, 11-19.

Shirakura O.,Yamada M., Hashimoto M., Ishimaru S., Takayama K., Nagai T. (1992) Effect of Amount and Composition of Granulating Solution on Physical Characteristics of Tablets, Drug Development and Industrial Pharmacy, 18(10) 1099-1110. 
Sienkiewicz G., Lausier J.L., Rhodes C.T. (1991) Recent Advances in Pharmaceutical Mixing Technology, Proceedings of the Interphex 1991 Technical Program, Cahners, 185192.

Skelly J.P., Van Buskirk G.A., Savello D.R., Amidon G.L., Arbit H.M., Dighe S., Fawzi M.B., Gonzalez M., Malick A.W., Malinowski H., Nedich R., Peck G.E., Pearce D.M.,Shah V., Shangraw R.F., Schwartz J.B., Truelove J. (1993) Scale-Up of Immediate Release Oral Solid Dosage Forms, Pharmaceutical Research, 10(2) 313-316.

Skelly J.P., Van Buskirk G.A., Amidon G.L., Arbit H.M., Augsburger L., Barr W.H., Berge S., Clevenger J., Dighe S., Fawzi M.B., Fox D., Gonzalez M., Gray V.A., Hoiberg C., Leeson L.J., Lesko L., Malinowski H., Nedich R., Nixon P.R., Pearce D.M., Peck G.E., Porter S., Robinson J., Savello D.R., Shah V., Shangraw R., Schwartz J.B., Schwartz P., Theeuwes F., and Wheatley T. (1993) Scaleup of Oral Extended-Release Dosage Forms, Pharmaceutical Research, 10(12) 1800-1811.

Staniforth J.N. (1988) Particle Engineering and Excipient Performance, Indian Journal of Pharmaceutical Sciences, 50(4), 213-220.

Swarbrick J., Boylan J.C., ed (1988) Encyclopedia of Pharmaceutical Technology, Marcel Dekker, New York.

Thwaites P.M., Mashadi A.B., Moore W.D. (1991) An Investigation of the Effect of High Speed Mixing on the Mechanical and Physical Properties of Direct Compression Lactose, Drug Development and Industrial Pharmacy, 17(4),503-517. 
Timko R.J., Barrett J.S., McHUgh P.A., Chen S.T., Rosenberg H.A. (1987) Use of a Motor Load Analyzer to Monitor the Granulation Process in a High Intensity Mixer, Drug Development and Industrial Pharmacy, 13(3), 403-435.

Usteri M., Leuenberger H. (1989) Agglomeration of Binary Mixtures in a High-Speed Mixer, International Journal of Pharmaceutics, 55, 135-141.

Vecchio C., Bruni G., Gazzaniga A. (1994) Preparation of Indobufen Pellets by Using Centrifugal Rotary Fluidized Bed Equipment Without Starting Seeds, Drug Development and Industrial Pharmacy, 20(12), 1943-1956.

Veesler S., Boistelle R., Delacourte A., Guyot J.C., Guyot-Hermann A.M. (1992) Influence of Structure and Size of Crystalline Aggregates on Their Compression Ability, Drug Development and Industrial Pharmacy, 18(5), 539-560.

Vogel S.T. (1984) Directly Compressible Acetaminophen Granulation, United States Patent \# 4,439,453.

Vojnovic D., Moneghini M., Rubessa F. (1994) Optimization of Granulates in a High Shear Mixer by Mixer Design, Drug Development and Industrial Pharmacy, 20(6),10351047.

Vojnovic D., Rubessa F., Moneghini M., Zanchetta A. (1993) Simultaneous Optimization of Several Response Variables in a Granulation Process, Drug Development and Industrial Pharmacy, 19(12), 1479-1496. 
Vojnovic D., Selenati P., Rubessa F., Moneghini M., Zanchetta A. (1992) Wet Granulation in a Small Scale High Shear, Drug Development and Industrial Pharmacy, 18(9), 961-972.

Wan L.S.C., Heng P.W.S., Liew C.V. (1994) The Role of Moisture and Gap Air Pressure in the Formation of Spherical Granules by Rotary Processing, Drug Development and Industrial Pharmacy, 20(16), 2551-2561.

Woodruff C.W., Nuessle N.O. (1972) Effect of Processing Variables on Particles Obtained by Extrusion-Spheronization, Journal of Pharmaceutical Sciences, 61(5),787790. 